\title{
Leitlinie der Deutschen Atemwegsliga und der Deutschen Gesellschaft für Pneumologie und Beatmungsmedizin zur Diagnostik und Therapie von Patienten mit chronisch obstruktiver Bronchitis und Lungenemphysem (COPD)
}

\author{
Guidelines for the Diagnosis and Therapy of COPD Issued by Deutsche Atemwegsliga \\ and Deutsche Gesellschaft für Pneumologie und Beatmungsmedizin
}

Autoren

Institute
C. Vogelmeier ${ }^{1}$, R. Buhl, C. P. Criée, A. Gillissen, P. Kardos, D. Köhler, H. Magnussen, H. Morr,

D. Nowak, D. Pfeiffer-Kascha, W. Petro, K. Rabe, K. Schultz, H. Sitter, H. Teschler, T. Welte,

R. Wettengel, H. Worth ${ }^{2}$

Klinik für Innere Medizin mit Schwerpunkt Pneumologie · Universitätsklinikum Gießen und Marburg, Standort Marburg

Medizinische Klinik I, Klinikum Fürth
Bibliografie

DOI 10.1055/s-2007-959200

Online-Publikation: 13. 4. 2007

Pneumologie 2007; 61; e1-e40

(c) Georg Thieme Verlag KG

Stuttgart · New York

ISSN 0934-8387

Korrespondenzadresse

Prof. Dr. med.

\section{Claus Vogelmeier}

Klinik für Innere Medizin mit

Schwerpunkt Pneumologie, Universitätsklinikum Gießen und Marburg, Standort Marburg

Baldingerstraße

35043 Marburg

Claus.Vogelmeier@med.uni-

marburg.de

oder

Prof. Dr. med. Heinrich Worth

Medizinische Klinik I,

Klinikum Fürth

Jakob-Henle-Str.

90766 Fürth

med1@klinikum-fuerth.de

\begin{tabular}{|c|c|c|c|}
\hline Inhalt & Seite & Inhalt & Seite \\
\hline Vorwort & e2 & Therapie & e11 \\
\hline Ziele und Anwendungsbereich der Leitlinie & e2 & Prävention & e11 \\
\hline Entwicklungsprozess der Leitlinie & e2 & Leitsätze zur Prävention & e11 \\
\hline Klinische Algorithmen & e3 & Prävention des Tabakrauchens & e12 \\
\hline Sponsoring & e3 & Verzicht auf Tabakrauchen & e12 \\
\hline \multirow{2}{*}{$\begin{array}{l}\text { Strategien zur Förderung der Verbreitung } \\
\text { und Implementierung der Leitlinie }\end{array}$} & \multirow[t]{2}{*}{ e3 } & Raucherentwöhnung & e12 \\
\hline & & \multirow{2}{*}{$\begin{array}{l}\text { Prophylaxe von arbeitsplatzbezogenen } \\
\text { Schadstoffen }\end{array}$} & \multirow[t]{2}{*}{ e13 } \\
\hline Weiterentwicklung der COPD-Leitlinie & e4 & & \\
\hline Definitionen & e4 & Schutzimpfungen & e13 \\
\hline Epidemiologie und sozioökonomische & \multirow[t]{2}{*}{ e4 } & Influenzaschutzimpfung & e13 \\
\hline Bedeutung & & Influenzaprophylaxe & e13 \\
\hline Verlauf der Erkrankung & e5 & Pneumokokkenschutzimpfung & e13 \\
\hline Management der COPD & e5 & Langzeitbehandlung der stabilen COPD & e13 \\
\hline Diagnostik & e5 & Leitsätze & e13 \\
\hline Leitsätze & e5 & Bronchodilatatoren & e14 \\
\hline Untersuchungsmethoden & e5 & Anticholinergika & e15 \\
\hline Anamnese & e5 & $\beta_{2}$-Sympathomimetika & e15 \\
\hline Körperliche Untersuchung & e6 & Theophyllin & e15 \\
\hline Lungenfunktionsdiagnostik & e6 & Kombinationen von Bronchodilatatoren & e16 \\
\hline \multirow{2}{*}{$\begin{array}{l}\text { Reversibilitätstest mit Bronchodilatato- } \\
\text { ren }\end{array}$} & \multirow[t]{2}{*}{ e6 } & Inhalation mit Verneblern & e16 \\
\hline & & Glukokortikoide & e16 \\
\hline \multirow{3}{*}{$\begin{array}{l}\text { Reversibilitätstest mit Glukokortiko- } \\
\text { iden zur differenzialdiagnostischen Ab- } \\
\text { grenzung zwischen Asthma und COPD }\end{array}$} & \multirow[t]{3}{*}{ e7 } & Inhalative Glukokortikoide & e16 \\
\hline & & Systemische Glukokortikoide & e16 \\
\hline & & Kombinationen aus langwirksamen & e17 \\
\hline Weitere Lungenfunktionstests & e7 & \multicolumn{2}{|l|}{$\beta_{2}$-Sympathomimetika und Glukokortikoiden } \\
\hline Blutgasanalyse & e7 & Mukopharmaka & e17 \\
\hline CO-Diffusionskapazität & e7 & Immunmodulatoren & e17 \\
\hline Belastungstests & e7 & Antitussiva & e18 \\
\hline Röntgenaufnahme der Thoraxorgane & e7 & Atemstimulanzien & e18 \\
\hline Computertomographie des Thorax & e7 & Analgetika (Morphin) & e18 \\
\hline Elektrokardiogramm & e8 & Weitere medikamentöse Therapie- & e18 \\
\hline Echokardiographie & e8 & optionen & \\
\hline Laboruntersuchungen & e8 & Substitutionstherapie bei Alpha-1- & e18 \\
\hline Sputumdiagnostik & e8 & Protease-Inhibitor-Mangel & \\
\hline \multirow{2}{*}{$\begin{array}{l}\text { Diagnostisches Vorgehen bei Verdacht auf } \\
\text { COPD }\end{array}$} & \multirow[t]{2}{*}{ e8 } & Behandlung der Osteoporose & e18 \\
\hline & & Nichtmedikamentöse Therapie & e18 \\
\hline Schweregradeinteilung der stabilen COPD & e8 & Körperliches Training & e18 \\
\hline Leichtgradige COPD (Schweregrad I) & e8 & Patientenschulung & e19 \\
\hline Mittelgradige COPD (Schweregrad II) & e8 & Physiotherapie & e19 \\
\hline Schwere COPD (Schweregrad III) & e8 & Hilfsmittel zur Sekretelimination & e20 \\
\hline Sehr schwere COPD (Schweregrad IV) & e9 & Ernährung & e20 \\
\hline Differenzialdiagnosen & e10 & Pneumologische Rehabilitation & e21 \\
\hline Weitere differenzialdiagnostische Aspekte & e10 & Komponenten der pneumologischen & e21 \\
\hline Verlaufsuntersuchungen & e11 & Rehabilitation & \\
\hline
\end{tabular}




\begin{tabular}{|c|c|}
\hline nhalt & Se \\
\hline $\begin{array}{l}\text { Effekte der pneumologischen Rehabilitation bei Patienten } \\
\text { mit COPD }\end{array}$ & e21 \\
\hline Auswahl der Patienten & e21 \\
\hline Organisation der Pneumologischen Rehabilitation & e21 \\
\hline Langzeitsauerstofftherapie (LOT) & e22 \\
\hline Heimbeatmung & e22 \\
\hline Operative Therapieverfahren & e23 \\
\hline Bullektomie & e23 \\
\hline Lungenvolumenreduktion & e23 \\
\hline Lungentransplantation & e23 \\
\hline Palliative Therapie & e24 \\
\hline Management akuter Exazerbationen & e25 \\
\hline Leitsätze & e25 \\
\hline Definition & e25 \\
\hline Diagnostik & e25 \\
\hline Therapie der Exazerbation & e26 \\
\hline Medikamentöse Therapie & e27 \\
\hline Bronchodilatatoren & e27 \\
\hline
\end{tabular}

\section{Vorwort}

Der folgende Text stellt eine Überarbeitung der Leitlinie der Deutschen Atemwegsliga und der Deutschen Gesellschaft für Pneumologie von 2002 [1] dar. Diese ist notwendig geworden, da für alle wesentlichen Teilaspekte der COPD - Diagnostik, medikamentöse und nicht medikamentöse Therapie der stabilen COPD, Management der Exazerbationen, operative Verfahren in den letzten Jahren relevante Arbeiten erschienen sind und überarbeitete [2] bzw. neue internationale Leitlinien veröffentlicht wurden [3,4].

In Anlehnung an internationale Leitlinien [5-10] wird der Begriff COPD (chronic obstructive pulmonary disease) benutzt, der chronisch obstruktive Bronchitis, Lungenemphysem und deren Kombinationen umfasst, das Asthma hingegen ausschließt. Die Schweregradeinteilung wurde entsprechend der aktuellen GOLD-Leitlinie [2,5] überarbeitet. In der vorliegenden Version werden die wesentlichen diagnostischen Verfahren dargestellt. Neben neuen Erkenntnissen zur medikamentösen Behandlung werden weitere Therapieansätze (nichtinvasive Beatmung, operative Behandlungsverfahren des Lungenemphysems und Lungentransplantation) berücksichtigt. Weiter werden präventive Maßnahmen (Raucherentwöhnung, Schutzimpfung, Patientenschulung), Physiotherapie und die pneumologische Rehabilitation entsprechend ihrer Bedeutung erläutert.

\section{Ziele und Anwendungsbereich der Leitlinie}

Die vorliegende Leitlinie soll dazu beitragen, dass COPD-Patienten wissenschaftlich begründete, angemessene, wirtschaftliche und qualitätsgesicherte Verfahren der Diagnostik, Prävention und Behandlung unter Einschluss der Rehabilitation zuteil werden. Sie soll allen Ärzten (Allgemeinärzten, Internisten und Pneumologen in Praxis und Klinik) eine Hilfe für Diagnostik, Verlaufskontrolle und adäquate Therapie ihrer Patienten sein. Die Leitlinie ersetzt das Urteil des behandelnden Arztes nicht; sie soll vielmehr als Entscheidungshilfe für ein optimiertes Management der Patienten mit COPD genutzt werden.

Eine ausführliche Version enthält wesentliche Literaturangaben und Hinweise auf die Evidenz der dargestellten Aussagen. Eine Kurzversion mit praktikablen Schemata wird dem Arzt, der sich rasch orientieren will, bei Therapieentscheidungen zur Verfü-

\begin{tabular}{|l|l|}
\hline Inhalt & Seite \\
\hline Antibiotika & e27 \\
\hline $\begin{array}{l}\text { Glukokortikoide } \\
\text { Zusätzliche Maßnahmen bei der Behandlung akuter Exa- } \\
\text { zerbationen im Krankenhaus }\end{array}$ & e28 \\
\hline Beatmung & e29 \\
\hline$\quad$ Nichtinvasive Beatmung mit positivem Druck (NIPPV) & e29 \\
\hline $\begin{array}{l}\text { Invasive Beatmung } \\
\text { Management nach der akuten Exazerbation im Krankenhaus }\end{array}$ & e30 \\
\hline Exazerbationsprophylaxe & e30 \\
\hline Appendix A: & e31 \\
\hline Berufsbedingte Bronchitis & e31 \\
\hline Bronchitis im Unfall- und Berufskrankheitenrecht & e31 \\
\hline $\begin{array}{l}\text { Bronchitis im sozialen Entschädigungsrecht und im Schwer- } \\
\text { behindertenrecht }\end{array}$ & e31 \\
\hline $\begin{array}{l}\text { Prävention am Arbeitsplatz } \\
\text { Appendix B: }\end{array}$ & e31 \\
\hline Teilnehmerliste der Konsensus-Konferenz & e32 \\
\hline Literaturverzeichnis & e32 \\
\hline
\end{tabular}

gung stehen. Eine Fassung in patientengerechter Sprache soll dem betroffenen Patienten eine aktive Mitarbeit bei der Bewältigung seiner chronischen Krankheit ermöglichen.

\section{Entwicklungsprozess der Leitlinie}

Die Leitlinie zur Behandlung von Patienten mit chronisch obstruktiver Bronchitis und Lungenemphysem (COPD) ist entsprechend den methodischen Vorgaben zur Entwicklung von Leitlinien für Diagnostik und Therapie der Arbeitsgemeinschaft der wissenschaftlichen medizinischen Fachgesellschaften (AWMF) $[11,12]$ erstellt worden und entspricht nach dem 3-Stufen-Konzept der AWMF einer $\mathrm{S}_{2}$-Leitlinie, ergänzt durch klinische Algorithmen zur Diagnostik und Therapie der COPD. Die 3 Stufen der AWMF-Leitlinien $\left(S_{1}, S_{2}, S_{3}\right)$ lassen sich folgendermaßen charakterisieren:

$\mathrm{S}_{1}$ (Expertengruppe): Eine repräsentativ zusammengesetzte Expertengruppe der Wissenschaftlichen Medizinischen Fachgesellschaft erarbeitet im informellen Konsens eine Leitlinie, die vom Vorstand der Fachgesellschaft verabschiedet wird.

$\mathrm{S}_{2}$ (formale Konsensusfindung): Vorhandene Leitlinien der Stufe 1 werden in bewährten formalen Konsensusverfahren beraten und modifiziert und als Leitlinien der Stufe 2 verabschiedet.

$\mathrm{S}_{3}$ (Leitlinie mit allen Elementen systematischer Erstellung): Der formale Konsensusprozess wird durch weitere systematische Elemente ergänzt: logische Analyse (klinischer Algorithmus), formale Konsensusfindung, Evidenzbasierung, Entscheidungsanalyse und Outcome-Analyse.

Der Konsens ist notwendig, um bei geringer vorhandener Evidenz Akzeptanz für eine Leitlinie zu erzeugen und die Verbreitung und Implementierung zu unterstützen. Evidenzbasierung bedeutet die Einbeziehung von Metaanalysen, klinischen Studien und epidemiologischen Untersuchungen, um sie für Entscheidungen beim individuellen Patienten nutzbar zu machen.

Die Entscheidungs- und Outcomeanalyse berücksichtigen Modelle mit probabilistischen Entscheidungsbäumen, erwartetem Nutzen und ökonomischen Aspekten sowie die Bestimmung des Gesundheitsstatus (ermittelt durch den Arzt) und der Lebensqualität (Selbstbeurteilung von Patienten in einem validierten Fragebogen). Die resultierende Leitlinie soll einfach und klar, aber auch umfassend sein. 


\section{Klinische Algorithmen}

Ein klinischer Algorithmus ist ein in unendlich vielen Schritten formuliertes Verfahren zur Lösung eines klinischen Problems unter Benutzung von bedingten logischen Anweisungen (WennDann-Logik).

Die Darstellung erfolgt üblicherweise in graphischem Format mit einer von der Society for Medical Decision Making [13] empfohlenen Standardnomenklatur ( Abb. 1). Dabei unterscheidet man Zustands-, Aktions- und Entscheidungsknoten. Zustandsund Aktionsknoten haben je einen Ausgang, Entscheidungsknoten haben genau zwei Ausgänge (ja und nein).

Grundlage der Leitlinie ist eine Bestandsaufnahme der vorhandenen internationalen und nationalen Empfehlungen sowie der verfügbaren Literatur zum Krankheitsbild mit Angaben zur Validität der gemachten Aussagen entsprechend den Vorgaben der Evidence Based Medicine ( Tab. 1). Im Text werden die Evidenzgrade entsprechend der Definition der GOLD-Leitlinie [2,5] angegeben.

Am 30.10.2005 fand in Kassel eine Konsensuskonferenz statt. Zu dieser Konferenz wurden alle an der Versorgung der COPD beteiligten Gruppierungen eingeladen. Die Teilnehmer sind in Appendix B genannt.

Während der Konferenz wurden für wesentliche Entscheidungsprozesse in Diagnostik und Therapie relevante Algorithmen entwickelt. Diese wurden in Expertenkreisen diskutiert und in einem weiteren Delphi-Prozess bis zur endgültigen Konsensusfindung bearbeitet.

Eine Rohfassung der Leitlinie wurde durch einige Mitglieder des Expertengremiums (C. Vogelmeier, H. Worth, P. Kardos, T. Welte, H. Magnussen) erstellt und von den weiteren Mitgliedern des Expertenkomitees kritisch kommentiert.

Eine verbesserte Fassung wurde analog einem Delphi-Verfahren allen Autoren sowie dem Vorstand der Deutschen Atemwegsliga mit der Bitte um eine Stellungnahme zugeleitet. Diesem Personenkreis gehören Pneumologen in Praxis und Klinik, aber auch Hausärzte an. Die Zusatzinformationen wurden durch den federführenden Autor zusammengefasst.

\section{Sponsoring}

Die Deutsche Atemwegsliga wurde von Ärzten mit dem Ziel gegründet, die Versorgung von Patienten mit Lungenkrankheiten in Deutschland zu verbessern. Ihre Arbeit erfolgt in Abstimmung mit der Deutschen Gesellschaft für Pneumologie und Beat-

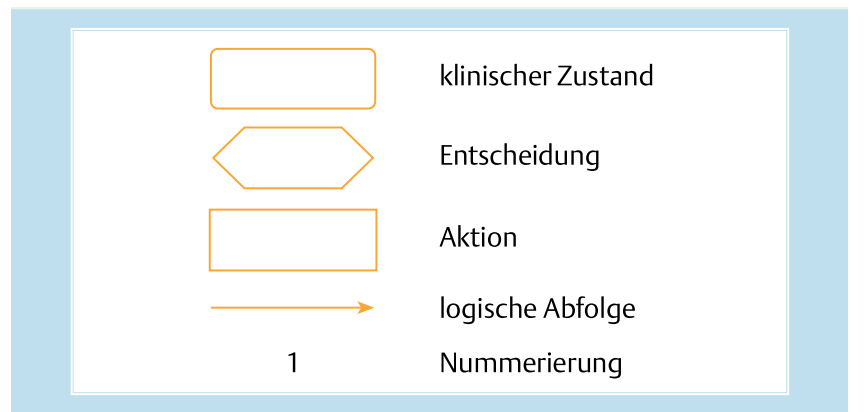

Abb. 1 Standardisierte Terminologie für klinische Algorithmen.

mungsmedizin. Die Mitglieder der Deutschen Atemwegsliga sind überwiegend Internisten und Ärzte für Allgemeinmedizin, zu einem geringeren Anteil auch Patienten und/oder deren Angehörige. Der Vorstand besteht aus in Praxis und Klinik tätigen Pneumologen sowie pneumologisch tätigen Kinderärzten. Die Geschäftsstelle der Deutschen Atemwegsliga wird aus Mitgliedsbeiträgen sowie aus Beiträgen eines Fördervereins finanziert. Dem Förderverein gehören 19 führende Pharma-Unternehmen sowie ein Gerätehersteller an. Kommentare von Repräsentanten der Firmen wurden bei der Entwicklung der Leitlinie in dem vom Vorstand der Atemwegsliga benannten unabhängigen Expertengremium diskutiert und bewertet.

Für einzelne Teilnehmer der Konsensuskonferenz wurden seitens der Deutschen Atemwegsliga auf Antrag Reisekosten erstattet.

Die Geschäftsstelle der Deutschen Atemwegsliga sorgte für den Versand der erstellten Fassungen der Leitlinien und organisierte die Konsensuskonferenz.

\section{Strategien zur Verbreitung und Implementierung der Leitlinie}

Die vorliegende Leitlinie zur Behandlung von Patienten mit COPD soll in den Fachzeitschriften für Allgemeinärzte, Internisten und Pneumologen publiziert werden. Außerdem kann die Leitlinie über die Geschäftsstelle der Deutschen Atemwegsliga bezogen werden. Hierdurch wird eine weite Verbreitung der Leitlinie unter den behandelnden Ärzten ermöglicht.

Begünstigt werden Verbreitung und Implementierung der Leitlinie durch das Angebot von Kurzversionen für Ärzte und für Pa-

Tab. 1 Beschreibung von Evidenz- und Empfehlungsgraden

\begin{tabular}{|c|c|c|c|c|}
\hline $\begin{array}{l}\text { Evidenzgrad } \\
\text { GOLD-Initiative }\end{array}$ & $\begin{array}{l}\text { Evidenzgrad } \\
\text { SIGN }\end{array}$ & $\begin{array}{l}\text { Empfehlungsgrad } \\
\text { SIGN }\end{array}$ & Quellen & Definition \\
\hline A & I & A & $\begin{array}{l}\text { randomisierte kontrollierte Studien } \\
\text { (RKS) mit großer Patientenzahl }\end{array}$ & $\begin{array}{l}\text { konsistente Daten von RKS mit großer } \\
\text { Patientenzahl }\end{array}$ \\
\hline B & II & A & RKS mit begrenzter Patientenzahl & $\begin{array}{l}\text { RKS mit geringerer Patientenzahl und } \\
\text { gewissen Inkonsistenzen } \\
\text { Meta-Analysen von RKS }\end{array}$ \\
\hline C & II oder III & B oder C & $\begin{array}{l}\text { nicht-randomisierte Studien, Beob- } \\
\text { achtungen }\end{array}$ & $\begin{array}{l}\text { Ergebnisse aus unkontrollierten oder } \\
\text { nicht randomisierten Studien oder aus } \\
\text { Beobachtungen }\end{array}$ \\
\hline D & IV & D & Expertenmeinung & $\begin{array}{l}\text { Stellungnahme anhand der Erfahrung } \\
\text { der Experten ohne eindeutige Studien- } \\
\text { ergebnisse wie bei A-C }\end{array}$ \\
\hline
\end{tabular}

SIGN: Scottish Intercollegiate Guideline Network [11] 
tienten, die über die Geschäftsstelle der Deutschen Atemwegsliga beziehbar sind.

Alle Leitlinien können über das Internet eingesehen werden. Eine weitere Implementierung soll dadurch erfolgen, dass die Leitlinie Grundlage der strukturierten Schulungsprogramme für Patienten mit COPD wird.

Die Trainerseminare für die Schulung von COPD-Patienten erfolgen mit strukturierten Schulungsprogrammen auf der Basis der nationalen COPD-Leitlinie. Sie werden ebenfalls von der Deutschen Atemwegsliga angeboten. In überregionalen und regionalen Fortbildungen wird die COPD-Leitlinie diskutiert. Sie soll ferner in größere Netzwerke niedergelassener Ärzte implementiert und in Disease Management Programmen genutzt werden.

\section{Weiterentwicklung der COPD-Leitlinie}

Verantwortlich für die Aktualisierung der Leitlinie ist der Vorstand der Deutschen Atemwegsliga. Ein Update der COPD-Leitlinie ist immer dann geplant, wenn wesentliche neue wissenschaftliche Erkenntnisse zu berücksichtigen sind, spätestens jedoch nach 5 Jahren.

\section{Definitionen}

Die meisten Patienten mit chronischem Husten und Auswurf entwickeln keine obstruktive Lungenerkrankung, sondern leiden an einer nicht obstruktiven chronischen Bronchitis („simple chronic bronchitis“).

Nach der WHO-Definition [14] liegt eine chronische Bronchitis vor, wenn Husten und Auswurf über wenigstens 3 Monate in mindestens 2 aufeinander folgenden Jahren bestehen.

Das Lungenemphysem wird pathologisch-anatomisch definiert als irreversible Erweiterung und Destruktion der Lufträume distal der terminalen Bronchiolen [15].

Es geht in der Regel mit einer funktionell relevanten Atemwegsobstruktion einher. Zwischen dem Ausmaß der Destruktion im alveolären Bereich und der messbaren Atemwegsobstruktion besteht eine schlechte Korrelation. Beim Lungenemphysem sind die Gasaustauschfläche und die Elastizität vermindert.

Die COPD ist eine Erkrankung, die verhindert und behandelt werden kann. Sie ist charakterisiert durch eine Atemwegsobstruktion, die nicht vollständig reversibel ist. Die Atemwegsobstruktion ist typischerweise progredient. Sie ist assoziiert mit einer abnormen Entzündungsreaktion, die durch Partikel oder Gase ausgelöst und in erster Linie durch Zigarettenrauch verursacht wird. Die COPD betrifft nicht nur die Lunge, sondern hat auch signifikante extrapulmonale Effekte, die zum Schweregrad der Erkrankung beitragen können.

Abzugrenzen von der Diagnose COPD sind andere Ursachen einer chronischen Atemwegsobstruktion, wie Asthma, Mukoviszidose, Bronchiektasie, diffuse Lungenparenchymerkrankungen mit Obstruktion (Sarkoidose) und Bronchiolitis obliterans. Die Einteilung von COPD-Patienten in Patientengruppen mit chronisch obstruktiver Bronchitis mit oder ohne Lungenemphysem ist mit einfachen Kenngrößen der Lungenfunktion häufig schwierig, insbesondere in Frühstadien. In fortgeschrittenen Stadien der Erkrankung erscheint die Unterscheidung zwischen chronisch obstruktiver Bronchitis und Lungenemphysem mittels differenzierter Lungenfunktionsanalyse und bildgebender Diagnostik auch im Hinblick auf die optimale Nutzung vorhandener
Therapieoptionen sinnvoll. Klinisch bedeutsam ist die Differenzierung zwischen COPD und Asthma, da Ursachen, Behandlung und Prognose beider Krankheitsbilder unterschiedlich sind.

\section{Epidemiologie und sozioökonomische Bedeutung}

Valide Angaben zur Prävalenz der COPD in Deutschland liegen gegenwärtig noch nicht in publizierter Form vor.

Die Prävalenz der chronischen Bronchitis wird bei der erwachsenen Bevölkerung in Deutschland auf 10 - 15\% [16] geschätzt. Der Anteil der chronisch obstruktiven Bronchitis - Husten, Auswurf und Atemwegsobstruktion - an der Gesamtprävalenz ist nicht genau bekannt.

In den offiziellen deutschen Sterbestatistiken nimmt die chronische Bronchitis mit 10000 Männern und Frauen im Jahr [17] keinen vorderen Platz ein. Da sich diese Zahlen aber auf globale Angaben aus den Totenscheinen und den ICD 9-Ziffern 490 (Bronchitis, nicht als akut oder chronisch bezeichnet) und 491 (Chronische Bronchitis) beziehen, ist eine erhebliche Unterschätzung der Mortalität der Bronchitis zu unterstellen.

Weltweit ist die COPD gegenwärtig die vierthäufigste Todesursache. Für die nächsten Jahrzehnte ist ein weiterer Anstieg von Prävalenz, Morbidität und Mortalität zu erwarten [18,19], so dass die COPD im Jahre 2020 unter den häufigsten Todesursachen auf den 3. Platz und bezüglich der Krankheitsfolgen - gemessen an der Summe aus den Jahren, die durch vorzeitigen Tod verloren gegangen sind, und aus den Jahren, die mit einer schweregradgewichteten Behinderung (DALY: disability adjusted life years) gelebt wurden - von Rang 12 auf Rang 5 der 15 weltweit häufigsten Erkrankungen vorrücken wird.

Unbestritten ist die enorme sozioökonomische Bedeutung der COPD [20,21]. Krankenhausstatistiken weisen seit 1996 für alle obstruktiven Atemwegserkrankungen 2,7 Mio. Krankenhaustage in Deutschland auf, der weitaus größte Teil dürfte zu Lasten der chronischen Bronchitis und ihrer Folgen gehen. Hochgerechnet aus den Angaben der AOK, verursacht die chronische Bronchitis jährlich etwa 25 Mio. Arbeitsunfähigkeitstage [16]; die volkswirtschaftlichen Gesamtkosten betragen, zurückhaltenden Schätzungen zufolge, etwa 5,93 Mrd. € [16]. Eine andere Studie schätzt die jährlichen Gesamtkosten der COPD in Deutschland auf 5,47 Mrd. €[22].

Nach einer prospektiven Krankheitskostenstudie an 785 COPDPatienten fallen hochgerechnet direkte Kosten von 4,50 Mrd. $€$ und indirekte Kosten von 3,94 Mrd. $€$ durch die COPD in Deutschland an. Den größten Anteil der direkten Kosten nehmen hierbei mit 41,4\% die Arzneimittelkosten ein, gefolgt von den Kosten für Hospitalisierung mit 31,6\% und den Kosten für ärztliche Leistungen mit 20,6\% [23].

Bei den indirekten Kosten bildet die Arbeitsunfähigkeit mit einem Anteil von 45,8\% den größten Kostenblock, gefolgt von den Pflegekosten mit 21,7\%.

Die durchschnittlichen jährlichen Kosten in Deutschland pro COPD-Patient aus volkswirtschaftlicher Sicht wurden auf $3027 €$ geschätzt, wobei Krankenhausaufenthalte mit 26\%, Medikamente mit $23 \%$, die Frührente mit $17 \%$ und die Rehabilitation mit 1,5\% zu diesen Kosten beitragen. Für die gesetzlichen Krankenversicherungen lagen die Kosten pro Patient bei $1944 €$ [24]. 


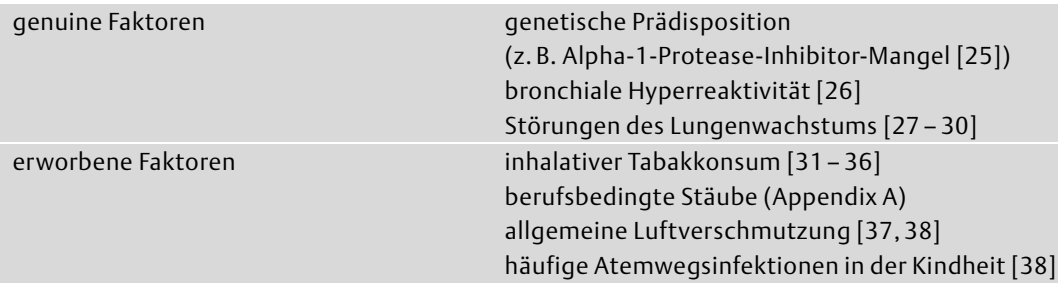

\section{Verlauf der Erkrankung}

Der Krankheitsverlauf der COPD ist durch eine progrediente Verschlechterung der Lungenfunktion und eine zunehmende Beeinträchtigung des Befindens, der Leistungsfähigkeit und der Lebensqualität gekennzeichnet, insbesondere hervorgerufen durch rezidivierende Exazerbationen und zunehmende Auswirkungen auf andere Organe.

\section{Management der COPD}

Wesentlich für das Management der COPD [2,5] sind

1. eine exakte Diagnose als Grundlage einer effektiven und differenzierten Therapie,

2. präventive Maßnahmen, insbesondere die Ausschaltung von Risikofaktoren,

3. die Langzeittherapie,

4. die Behandlung akuter Exazerbationen.

Ziele eines effektiven Managements sind

Verminderung der Progression der Erkrankung,

Symptomlinderung,

Steigerung der körperlichen Belastbarkeit,

Verbesserung des Gesundheitsstatus und der Lebensqualität, Vorbeugung und Behandlung von Exazerbationen,

Vorbeugung und Behandlung von Komplikationen,

Reduktion der Mortalität.

Die Frühdiagnostik der COPD wird dadurch erschwert, dass Patienten mit leicht- oder sogar mittelgradiger COPD die progrediente Belastungsdyspnoe infolge körperlicher Schonung häufig nicht als störend empfinden und sich daher einer für die Besserung der Prognose möglicherweise wichtigen Frühdiagnostik und frühzeitigen Therapie entziehen.

Der Nutzen von lungenfunktionsanalytischen Screening-Untersuchungen in der Allgemeinbevölkerung oder bei Rauchern ist allerdings nicht gesichert. Wesentlich ist bei der Angabe von chronischem (morgendlichem) Husten und/oder Auswurf und/ oder Belastungsdyspnoe sowie bei Vorliegen von Risikofaktoren ( Tab. 2) die Verdachtsdiagnose „COPD“ zu stellen und die entsprechende Diagnostik einzuleiten.

Bei jedem Schweregrad und in Abhängigkeit von der Komorbidität müssen die Therapieziele individuell und realistisch festgelegt werden. Bei Erstellung eines individuellen Therapieplanes sind Nutzen und Risiken der Behandlungsmaßnahmen für den Betroffenen sowie die direkten und die indirekten Kosten zu beachten.

Prinzipiell ist nach erreichter Besserung der Symptomatik für COPD-Patienten eine Reduktion der Therapie zu erwägen. Infolge der typischerweise progredienten Verschlechterung des Befindens und der Lungenfunktion ist allerdings häufig eine Intensivierung der medikamentösen und nichtmedikamentösen Therapiemaßnahmen notwendig.
Wesentlich sind auch die Vorbeugung und frühzeitig einsetzende, adäquate Behandlung akuter Exazerbationen, um das Auftreten von Komplikationen und kostenintensive Hospitalisationen zu minimieren.

\section{Diagnostik}

\section{Leitsätze}

1. Die Diagnose der COPD basiert auf der Angabe charakteristischer Symptome, dem Vorliegen von Risikofaktoren und dem lungenfunktionsanalytischen Nachweis einer nicht vollständig reversiblen Atemwegsobstruktion. Wichtigster Risikofaktor in Deutschland ist das Zigarettenrauchen.

2. Bei Patienten mit chronischem Husten und Auswurf sollte eine Lungenfunktionsprüfung durchgeführt werden, auch dann, wenn sie keine Atemnot verspüren.

3. Für die Diagnosestellung und für die Abschätzung des Schweregrades ist die Spirometrie die am besten validierte lungenfunktionsanalytische Methode.

4. Zur weiteren Differenzierung der Atemnot sowie zur Differenzierung von chronisch obstruktiver Bronchitis und Lungenemphysem haben sich die Ganzkörperplethysmographie sowie die Bestimmung der CO-Diffusionskapazität bewährt.

5. Bei allen Patienten mit einer ausgeprägten Belastungsdyspnoe, stark eingeschränkter $\mathrm{FEV}_{1}$ oder klinischen Zeichen einer Rechtsherzbelastung sollten eine Bestimmung der 6-Minuten-Gehstrecke sowie eine Blutgasanalyse in Ruhe und ggf. unter körperlicher Belastung durchgeführt werden. Die Diagnose COPD ist bei allen Patienten mit Husten, Auswurf, Atemnot und/oder Vorhandensein typischer Risikofaktoren ( Tab.2) in Erwägung zu ziehen. Die Diagnose wird durch den Nachweis einer nicht vollständig reversiblen Atemwegsobstruktion gesichert.

\section{Untersuchungsmethoden \\ Anamnese}

Chronischer Husten ist oft das Initialsymptom der COPD [39]. Er kann initial intermittierend, im Verlauf ständig morgens, später auch tagsüber vorhanden sein.

In vielen Fällen entwickelt sich eine Atemwegsobstruktion allerdings auch ohne Husten. Jede Form chronischen Auswurfs kann ein Indikator für eine COPD sein. Nicht jeder Patient mit Husten mit oder ohne Auswurf entwickelt jedoch eine COPD. Häufig suchen Patienten erst wegen Atemnot, die sich zunächst unter starker, bei Progression der Krankheit auch unter geringer Belastung bemerkbar macht, den Arzt auf. Thorakales Engegefühl und pfeifende Atemgeräusche können auch vorhanden sein.

Die Anamnese sollte bei Verdacht auf COPD folgende Angaben enthalten:

Exposition gegenüber Tabakrauch (aktiv: In Packungsjahren (pack-years) sowie passiv) und anderen Risikofaktoren

(- Tab. 2), 
Angaben über Asthma, Allergien, Sinusitiden, Nasenpolypen, Atemwegsinfekte und andere Atemwegserkrankungen, Lungenkrankheiten in der Familienanamnese,

Berufsanamnese,

Verlauf und Intensität der Symptomatik,

körperliche Belastbarkeit,

Häufigkeit und Schwere von Exazerbationen sowie frühere

Krankenhausaufenthalte,

Komorbidität (Herzerkrankungen u.a.),

gegenwärtige Medikation,

Beeinträchtigung im Alltag,

soziale Anamnese,

Möglichkeiten zur Reduktion/Beseitigung von Risikofaktoren, Störungen der Atmung im Schlaf.

\section{Körperliche Untersuchung}

Bei Patienten mit geringer Ausprägung der COPD kann der körperliche Untersuchungsbefund unauffällig sein.

Bei mittelschwerer Erkrankung können die Kennzeichen der Obstruktion mit verlängertem Exspirium, Giemen, Pfeifen und Brummen feststellbar sein wie auch eine Lungenüberblähung mit tief stehendem, wenig verschieblichem Zwerchfell und hypersonorem Klopfschall.

Die schwere COPD ist durch folgende anamnestische Angaben und körperliche Untersuchungsbefunde gekennzeichnet:

Zeichen der chronischen Lungenüberblähung mit abgeschwächtem Atemgeräusch, leisen Herztönen, Fassthorax und inspiratorischen Einziehungen im Bereich der Flanken, pfeifende Atemgeräusche, insbesondere bei forcierter Exspiration,

Zeichen der Sekretansammlung im Anhusteversuch,

zentrale Zyanose,

Konzentrationsschwäche und verminderte Vigilanz,

Gewichtsverlust,

periphere Ödeme,

indirekte Zeichen der pulmonalen Hypertonie mit präkordialen Pulsationen, betontem Pulmonalklappenschlusston, einer Tricuspidalklappeninsuffizienz mit einem Systolikum über dem 3. bzw. 4. ICR rechts parasternal.

Dabei ist anzumerken, dass nicht in jedem Fall alle genannten Symptome bzw. Befunde vorliegen.

\section{Lungenfunktionsdiagnostik}

In allen Verdachtsfällen und bei allen Schweregraden der COPD sowie zur Differenzialdiagnose der Dyspnoe sollte eine Lungenfunktionsanalyse durchgeführt werden. Die Diagnose COPD basiert auf der Feststellung einer Atemwegsobstruktion.

Der Nachweis kann mittels Spirometrie, Analyse von Fluss-Volumen-Diagrammen oder der Ganzkörperplethysmographie erfolgen.

Von den verfügbaren Kenngrößen der Obstruktion sind die Messung der 1-Sekunden-Kapazität (syn. Forciertes Einsekundenvolumen, $\mathrm{FEV}_{1}$ ), der inspiratorischen Vitalkapazität (VC) und die Bestimmung des Verhältnisses $\mathrm{FEV}_{1} / \mathrm{VC}$ die mit der höchsten Evidenz gesicherten Kenngrößen zur Charakterisierung der COPD und zur Beurteilung des natürlichen Verlaufs der Erkrankung (Evidenzgrad A).

Diese Leitlinie verwendet die VC für die Definition der Obstruktion, jetzt auch in Übereinstimmung mit der neuen Spirometrieempfehlung der Deutschen Atemwegsliga [40] und der ATS/ERSKonsensus Empfehlung zur Lungenfunktionsdiagnostik [41].
Es ist darauf hinzuweisen, dass GOLD die Obstruktion durch das Verhältnis von $\mathrm{FEV}_{1}$ zur forcierten Vitalkapazität (FVC) definiert $[2,5]$. In Grenzfällen können sich hierdurch Abweichungen ergeben.

Das Vorliegen einer Obstruktion wird vereinfachend, aber in Anlehnung an die meisten nationalen und internationalen Empfehlungen festgestellt, wenn der Quotient aus $\mathrm{FEV}_{1}$ und VC $\left(\mathrm{FEV}_{1} / \mathrm{VC}\right)<70 \%$ ist (Empfehlungsgrad A, Evidenzgrad D), obwohl der Wert eigentlich alters- und geschlechtsabhängig ist.

Normale Werte der $\mathrm{FEV}_{1} / \mathrm{VC}$ schließen die Diagnose COPD in der Regel aus, nicht jedoch die chronische, nicht obstruktive Bronchitis. Auch bei einzelnen Patienten mit einem Lungenemphysem, das an erhöhten Werten der funktionellen Residualkapazität (FRC) bzw. des intrathorakalen Gasvolumens (ITGV), einer Erniedrigung der CO-Diffusionskapazität (DLCO), häufig ausgeprägt verminderten Werten der maximalen Atemstromstärken nach Ausatmung von $50 \%$ und $75 \%$ der Vitalkapazität $\left(\mathrm{FEF}_{50}\right.$, $\mathrm{FEF}_{75}$ ) erkennbar ist, liegt keine Einschränkung der $\mathrm{FEV}_{1} / \mathrm{VC}$ vor. Neuere Untersuchungen belegen, dass durch Messungen der inspiratorischen Einsekundenkapazität $\left(\right.$ FIV $\left._{1}\right)$ [42] und der inspiratorischen Kapazität (IC) [43-45] wertvolle zusätzliche Informationen über die Überblähung der Lunge und die hierdurch bedingte funktionelle Beeinträchtigung des Patienten mit COPD gewonnen werden können, die mit dem Ausmaß der Dyspnoe besser korrelieren als die $\mathrm{FEV}_{1}$.

Die nach Inhalation von Bronchodilatatoren bestimmte $\mathrm{FEV}_{1}$ ist ein Prädiktor der Langzeitprognose $[46,47]$.

Die Messung der Peak-Flow-Werte ist für das Monitoring der COPD weniger geeignet als für das Asthma. Peak-Flow-Werte von mehr als $80 \%$ des Sollwertes schließen eine leichtgradige COPD nicht aus. Im Allgemeinen resultiert aus der Peak-FlowMessung eine Unterschätzung des Schweregrades der COPD. Bei Exazerbationen der COPD geht die Zunahme der Beschwerden dem Abfall der Peak-Flow-Werte voran [48].

\section{Reversibilitätstests mit Bronchodilatatoren}

Die Messung der Reaktion der Atemwegsobstruktion auf Bronchodilatatoren (raschwirksame $\beta_{2}$-Sympathomimetika und/ oder Anticholinergika) ist vor allem für die Differenzialdiagnose zwischen Asthma und COPD hilfreich.

Bei COPD-Patienten ist die akute Reaktion auf Bronchodilatatoren häufig bei Wiederholung des Tests nicht reproduzierbar [49] (Evidenzgrad B). Konsequenzen für die weitere Therapie bei Nichtansprechen ergeben sich nicht, da der Haupteffekt der Bronchodilatatoren bei der COPD in der Verminderung der Überblähung in Ruhe und der dynamischen Überblähung unter Belastung [43-45,50] (Evidenzgrad B) sowie in der Reduktion der Frequenz der Exazerbationen gesehen wird [51 -54] (Evidenzgrad B).

Die Untersuchungen sollten nach Möglichkeit in klinisch stabilem und infektfreiem Zustand des Patienten durchgeführt werden. Kurzwirksame $\beta_{2}$-Sympathomimetika sollten mindestens 6 Stunden, Anticholinergika 6-12 Stunden, langwirksame $\beta_{2}$-Sympathomimetika 12 Stunden, langwirksame Anticholinergika und retardierte Theophyllinpräparate 24 Stunden vor der Untersuchung abgesetzt werden.

Die Messungen der $\mathrm{FEV}_{1}$ erfolgen vor und 30 Minuten nach Inhalation eines kurzwirksamen $\beta_{2}$-Sympathomimetikums (z.B. bis zu $400 \mu \mathrm{g}$ Salbutamol oder bis zu $200 \mu \mathrm{g}$ Fenoterol oder $500 \mu \mathrm{g}$ Terbutalin) und/oder bis zu $160 \mu \mathrm{g}$ Ipratropiumbromid. Eine Kombination der Bronchodilatatoren wird empfohlen, um eine maximale Antwort zu erreichen (Evidenzgrad D). Ein Anstieg 
der $\mathrm{FEV}_{1}$ um mehr als $200 \mathrm{ml}$ und um mindestens $15 \%$ gegenüber dem Ausgangswert gilt als Kriterium einer reversiblen Atemwegsobstruktion und spricht eher für Asthma als für COPD. Die Grenzen der Reversibilität (200 ml und 15\%) wurden willkürlich festgelegt (Empfehlungsgrad A, Evidenzgrad D). Eine sichere Unterscheidung zwischen Asthma und COPD ist auch mit diesem Instrument nicht immer möglich, da bei einem lang bestehenden Asthma die volle Reversibilität verloren gehen kann und viele COPD-Patienten eine gewisse - allerdings nie vollständige - Reversibilität aufweisen.

Reversibilitätstests mit Glukokortikoiden zur differenzialdiagnostischen Abgrenzung zwischen Asthma und COPD Glukokortikoide können zeitlich limitiert für die Abgrenzung zwischen Asthma und COPD eingesetzt werden.

Die Reaktion der postbronchodilatatorischen FEV $_{1}$ auf Glukokortikoide in einer stabilen Phase der Erkrankung kann durch eine zweiwöchige orale Applikation von täglich 20-40 mg Prednisolon oder durch eine nebenwirkungsärmere $2 x$ tägliche Inhalation einer mindestens mittleren Dosis eines inhalativen Glukokortikoids über 4-6 Wochen ermittelt werden. Die Kriterien für eine positive Reaktion der $\mathrm{FEV}_{1}$ entsprechen denjenigen bei der Applikation von Bronchodilatatoren.

Asthmatiker reagieren auf diese Kurzzeittherapie mit Glukokortikoiden regelhaft deutlich, COPD-Patienten nur in 10-20\% [55]. In solchen Fällen kann das Vorliegen von COPD und Asthma an-

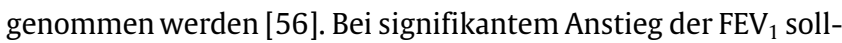
te der Patient mit inhalativen Glukokortikoiden behandelt werden.

\section{Weitere Lungenfunktionstests}

Bei Patienten mit geringer Obstruktion, geringem Dyspnoeempfinden und guter Leistungsfähigkeit ist die zusätzliche Bestimmung weiterer Kenngrößen der Lungenfunktion nicht indiziert. Bei Patienten mit Diskrepanz zwischen Dyspnoe und Einschränkung der $\mathrm{FEV}_{1}$, bei Patienten der Schweregrade III und IV (s. Schweregradeinteilung) oder bei Patienten, die nicht in der Lage sind, auswertbare forcierte Atemmanöver durchzuführen, sind neben der Spirometrie zusätzliche Messverfahren sinnvoll: Diese umfassen die Bestimmung der von der Mitarbeit des Patienten weniger abhängigen ganzkörperplethysmographischen Messgrößen (Raw: Atemwegswiderstand; ITGV: intrathorakales Gasvolumen; spezifische Resistance oder Conductance) zum Nachweis einer Obstruktion (Raw) und einer Überblähung (ITGV), die Bestimmung der arteriellen Blutgase in Ruhe und unter Belastung zum Nachweis einer latenten Störung des Gasaustausches und zur Beurteilung der Indikation einer Langzeitsauerstofftherapie sowie die Messung der CO-Diffusionskapazität zur Abschätzung der funktionellen Auswirkungen eines Lungenemphysems. Im Zusammenhang mit der Erfassung der Überblähung ist in letzter Zeit auch die Messung der inspiratorischen Kapazität (IC) und deren Verhältnis zur Totalkapazität (IC/TLC) in den Blickpunkt gerückt, nachdem gezeigt werden konnte, dass dieser Parameter mit der Mortalität [57] korreliert (Evidenzgrad B).

Wenn die Belastungsdyspnoe des Patienten nicht sicher ursächlich einzuordnen ist und/oder in ihrem Ausmaß nicht mit den Messwerten in Einklang steht, kann eine spiroergometrische Untersuchung weiteren Aufschluss bieten.

\section{Blutgasanalyse}

Eine arterielle Hypoxämie und eine Hyperkapnie werden bei Patienten mit schwerer COPD häufig angetroffen. Eine respiratorische Insuffizienz liegt bei $\mathrm{PaO}_{2}$-Werten $<60 \mathrm{~mm} \mathrm{Hg}$ mit oder ohne Hyperkapnie ( $\mathrm{PaCO}_{2} \geq 45 \mathrm{~mm} \mathrm{Hg}$ ) bei Atmung von Raumluft vor. In einem Präschock- oder Schockzustand muss die Blutgasanalyse über eine arterielle Punktion erfolgen, sonst wird hyperämisiertes Kapillarblut aus dem Ohrläppchen gewonnen.

Die Pulsoxymetrie ersetzt die direkte Analyse der arteriellen Blutgase nicht, insbesondere nicht bei klinischer Verschlechterung des Patienten oder beim Auftreten von Komplikationen, da sie keine Auskunft über den $\mathrm{CO}_{2}$-Partialdruck gibt. Die Pulsoxymetrie ist jedoch als Verlaufsparameter zur Kontrolle der Oxygenierung geeignet, da bei Werten über $90 \%$ eine Gefährdung durch eine kritische Hypoxämie auszuschließen ist [58]. Bei Werten zwischen $92 \%$ und 96\% ist infolge der Streuung der Methode eine zuverlässige Beurteilung einer Gasaustauschstörung erschwert.

\section{CO-Diffusionskapazität}

Die Bestimmung der CO-Diffusionskapazität wird üblicherweise nach der single-breath-Methode durchgeführt. Sie ist eine zur Analyse der Funktionseinschränkung beim Lungenemphysem wichtige Kenngröße [59], deren Messwerte mit dem pathologisch-anatomischen Schweregrad eines Lungenemphysems gut übereinstimmen [60-62].

\section{Belastungstests}

Kontrollierte Belastungstests können bei COPD-Patienten zur Differenzierung verschiedener Ursachen der Belastungsdyspnoe, zur Quantifizierung der eingeschränkten Belastbarkeit, zur Auswahl eines individuell abgestuften Trainingsprogramms und zur Beurteilung der Therapieeffekte von Medikamenten [44], wie auch von körperlichen Trainingsprogrammen in der pneumologischen Rehabilitation eingesetzt werden.

Für die Routinediagnostik bei Patienten im Stadium I und II sind sie entbehrlich. Die Auswahl der Belastungstests, der Belastungsprotokolle sowie der Kenngrößen zur Beurteilung der Belastbarkeit sind den Empfehlungen der Deutschen Gesellschaft für Pneumologie (DGP) zur Durchführung und Beurteilung von Belastungstests in der Pneumologie zu entnehmen [63].

\section{Röntgenaufnahmen der Thoraxorgane}

Eine Röntgenuntersuchung der Thoraxorgane ist bei der Erstdiagnostik sinnvoll und sollte in 2 Ebenen durchgeführt werden, damit Emphysemblasen identifiziert und bedeutsame weitere Erkrankungen, wie z.B. das Bronchialkarzinom oder eine Lungenstauung, erkannt werden können. Sie trägt zur Diagnose der COPD durch Ausschluss anderer Erkrankungen mit ähnlicher Symptomatik bei, ist aber nicht in der Lage, ein leichtgradiges Lungenemphysem zu erfassen [64].

\section{Computertomographie des Thorax}

Das hoch auflösende Computertomogramm des Thorax (HR-CT) kann zur Charakterisierung (zentrilobulär oder panlobulär), zur Quantifizierung und zur Beurteilung der Verteilung eines Lungenemphysems genutzt werden. Das HR-CT ist zur Charakterisierung ausgeprägter Lungenemphyseme insbesondere vor operativen Eingriffen, etwa der Lungenvolumenreduktion oder der Resektion von Bullae [65], notwendig. Bei Patienten mit COPD ohne Emphysemhinweis ist diese Untersuchung entbehrlich. 
Bei häufigen Exazerbationen ist das HR-CT hilfreich zum Nachweis von Bronchiektasen und beeinflusst damit die Entscheidung über den Einsatz von Antibiotika.

\section{Elektrokardiogramm}

Das EKG liefert Informationen über das Vorliegen einer koronaren Herzkrankheit und von Herzrhythmusstörungen, ist jedoch eine insensitive Methode zur Abschätzung einer Hypertrophie des rechten Ventrikels [66]. Gleichwohl können bei chronischem Cor pulmonale typische Zeichen der Rechtsherzbelastung gefunden werden. Ihr Fehlen schließt aber ein Cor pulmonale nicht aus.

\section{Echokardiographie}

Bei Verdacht auf ein Cor pulmonale erlaubt die Echokardiographie mit der Doppler- und Farbdopplertechnik häufig eine valide Abschätzung des systolischen Drucks im kleinen Kreislauf und der Dimensionen des rechten Ventrikels. Darüber hinaus kann die Echokardiographie wertvolle Dienste im Rahmen der Differenzialdiagnostik leisten, in dem sie u.a. die Bestimmung der systolischen linksventrikulären Pumpfunktion ermöglicht. Die Indikationen, die optimale Durchführung der Untersuchung und die geeigneten Kenngrößen zur Beurteilung eines Cor pulmonale und der pulmonalen Hypertonie sind den Empfehlungen der DGP zur Echokardiographie [67] zu entnehmen.

\section{Laboruntersuchungen}

Prinzipiell ist bei allen COPD-Patienten einmalig eine Untersuchung bezüglich eines Alpha-1-Protease-Inhibitor-Mangels empfehlenswert. Dies gilt in jedem Fall bei jüngeren ( $<50$ Jahre) sowie nichtrauchenden COPD-Patienten. Bei Exazerbationen ist die Bestimmung der BSG, des Blutbildes und des CRP im Serum sinnvoll. Bei Polyglobulie ist eine Blutgasanalyse indiziert.

\section{Sputumdiagnostik}

Die Indikationen für eine mikrobiologische Sputumdiagnostik sind im Kapitel „Exazerbationen“ dargestellt.

\section{Diagnostisches Vorgehen bei Verdacht auf COPD}

In $\bigcirc$ Abb. 2 ist ein Algorithmus zum Einsatz der genannten Verfahren in der Diagnostik und Differenzialdiagnostik der COPD sowie der Differenzierung zwischen chronisch obstruktiver Bronchitis und Lungenemphysem (Evidenzgrad C) dargestellt. Zur initialen Diagnostik, die vom niedergelassenen Allgemeinarzt oder Internisten durchgeführt werden kann, gehören die Anamnese, die körperliche Untersuchung, eine Thoraxaufnahme in 2 Ebenen und die Spirometrie. Reversibilitätstests mit Bronchodilatatoren bzw. Glukokortikoiden erlauben häufig die Differenzierung zwischen Asthma und COPD.

Wenn Atemnot angegeben wird, obwohl die Messwerte für absolute und relative Sekundenkapazität im Normbereich liegen, können die Ganzkörperplethysmographie (GKP) durch die Bestimmung des Atemwegswiderstandes (Raw) und des intrathorakalen Gasvolumens (ITGV) wertvolle Informationen liefern (Empfehlungsgrad A). So finden sich gelegentlich auch ohne $\mathrm{FEV}_{1}$-Erniedrigung erhöhte Werte des ITGV als Hinweis auf ein Lungenemphysem.

Auch die Reaktion auf Bronchodilatatoren kann insbesondere bei Patienten mit COPD und Lungenemphysem anhand der Änderung des ITGV besser beurteilt werden als mit der $\mathrm{FEV}_{1}[68]$.
Außerdem ist die ganzkörperplethysmographisch bestimmte

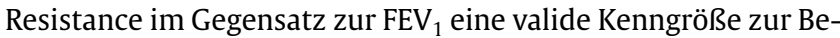
urteilung der Dauerbelastung der Atempumpe bei Patienten mit COPD [69]. Daher sollte der Patient mit einer chronischen Bronchitis und Atemnot bei normalen Werten der Spirometrie initial mindestens einmal ganzkörperplethysmographisch untersucht werden (Empfehlungsgrad A).

Die Analyse der arteriellen Blutgase wird zur Charakterisierung unklarer Atemnot unter Belastung eingesetzt, für ein adäquates Management der respiratorischen Insuffizienz bei COPD ist sie unentbehrlich (Empfehlungsgrad A).

Die Bestimmung der CO-Diffusionskapazität (DLCO) und des CO-Transfer-Koeffizienten (KCO) können zur Differenzierung zwischen chronisch obstruktiver Bronchitis und Lungenemphysem [70] beitragen (Evidenzgrad C, Empfehlungsgrad A).

Patienten mit - nach den genannten Untersuchungen - unklar gebliebener Dyspnoe müssen weiter diagnostiziert werden (Empfehlungsgrad A): Mit der Bronchoskopie lassen sich zentrale Atemwegsstenosen ausschließen. Systolische und diastolische Linksherzinsuffizienz, Anämie, pulmonale Hypertonie und Schlafapnoe können mit Echokardiogramm, Herzkatheter, Labor, CT, Angiographie, Ultraschall Dopplersonographie der peripheren Venen, Ventilations-Perfusions-Szintigraphie, Polysomnographie etc. abgeklärt werden.

\section{Schweregradeinteilung der stabilen COPD}

Eine einfache Schweregradeinteilung anhand der postbronchodilatatorisch gemessenen $\mathrm{FEV}_{1}$ soll als Orientierung für ein adäquates Management genutzt werden ( Tab.3). Die postbronchodilatatorische Messung dürfte geringeren Schwankungen als die präbronchodilatatorische unterliegen und eignet sich daher besser sowohl für die Schweregradeinteilung, als auch für die Langzeitbeobachtung des $\mathrm{FEV}_{1}$-Abfalls.

Anmerkung:

Für die Schweregradeinteilung gelten die Messwerte der $\mathrm{FEV}_{1}$ nach Bronchodilatation.

\section{Leichtgradige COPD (Schweregrad I)}

Der Schweregrad I ist durch eine Atemwegsobstruktion ohne signifikante $\mathrm{FEV}_{1}$-Verminderung charakterisiert $\left(\mathrm{FEV}_{1} / \mathrm{VC}<70 \%\right.$, aber $\mathrm{FEV}_{1} \geq 80 \%$ Soll). In der Regel bestehen chronischer Husten und Auswurf. Atemnot wird häufig noch nicht bemerkt. Bei diesem Schweregrad ist dem Patienten die Einschränkung der Lungenfunktion häufig nicht bewusst.

\section{Mittelgradige COPD (Schweregrad II)}

Der Schweregrad II ist durch eine Atemwegsobstruktion bei gleichzeitiger moderater $\mathrm{FEV}_{1}$-Verminderung ( $\mathrm{FEV}_{1}$-Werte zwischen $50 \%$ und weniger als $80 \%$ des Sollwertes) charakterisiert. Normalerweise ist dieses Stadium mit einer Zunahme der Symptome, insbesondere einer bei Belastung auftretenden Dyspnoe, verbunden.

\section{Schwere COPD (Schwergrad III)}

Patienten diesen Schweregrades sind durch eine höhergradige $\mathrm{FEV}_{1}$-Verminderung ( $\mathrm{FEV}_{1}$-Werte zwischen $30 \%$ und $50 \%$ Soll) gekennzeichnet. Das Ausmaß der Dyspnoe korreliert allerdings nicht oder nur schwach mit dem Schweregrad der Lungenfunktionseinschränkung [71], insbesondere im höheren Lebensalter [72]. 


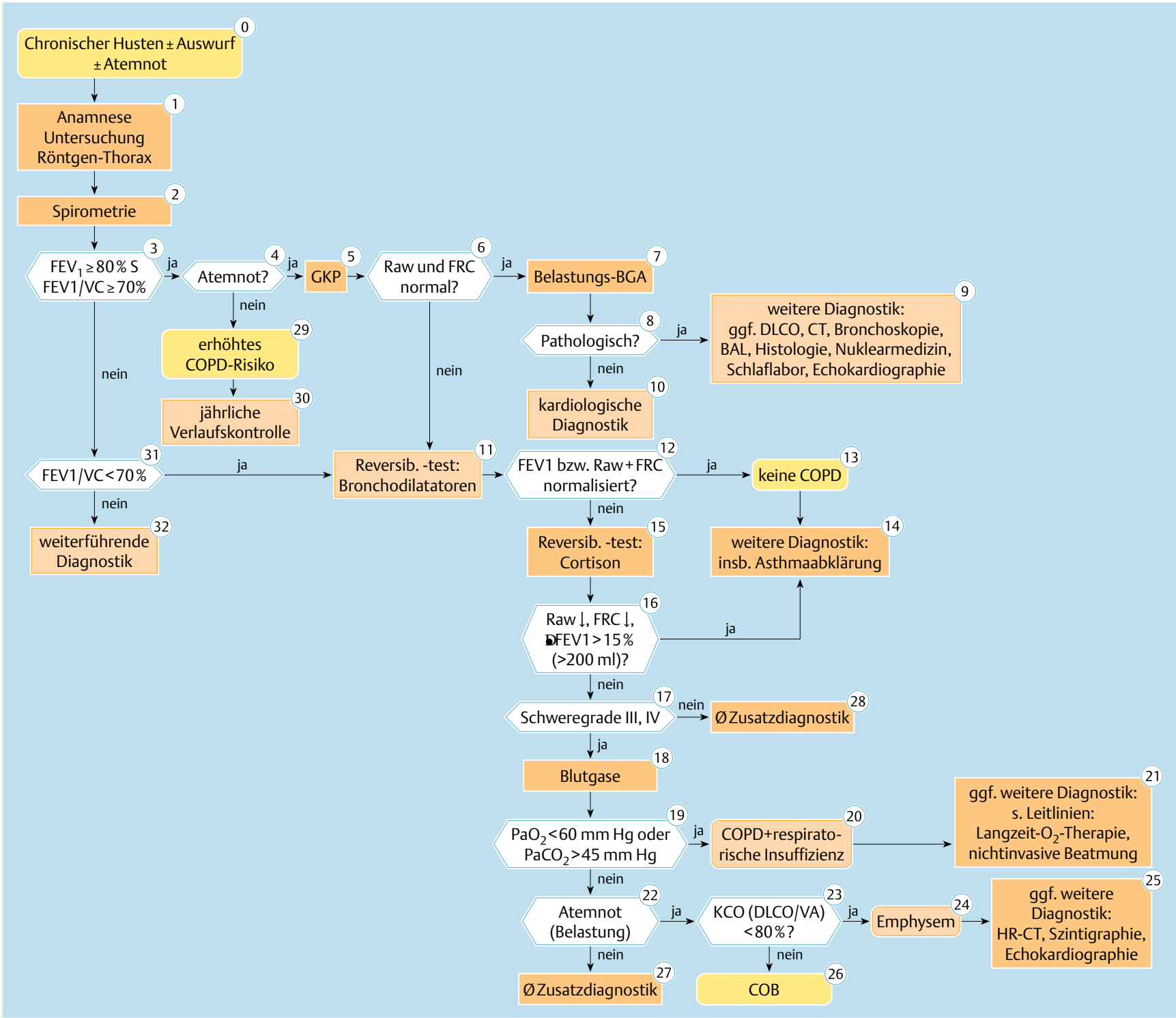

Abb. 2 Diagnostik der COPD

Der Algorithmus beschreibt die differenzierte Abklärung der COPD.

GKP: Ganzkörperplethysmographie, Raw: Gesamtatemwegswiderstand, FRC: funktionelle Residualkapazität, BGA: Blutgasanalyse, DLCO: CO-Diffusionskapazität, KCO: CO-Transferkoeffizient (DLCO/VA), VA: alveoläres Volumen, COB: chronisch obstruktive Bronchitis.

\begin{tabular}{|c|c|}
\hline Schweregrad & Kriterien \\
\hline I (leicht) & $\begin{array}{l}\mathrm{FEV}_{1} \geq 80 \% \text { Soll, }_{\mathrm{FEV}} / \mathrm{VC}<70 \% \\
\text { mit/ohne Symptomatik } \\
\text { (Husten, Auswurf) }\end{array}$ \\
\hline II (mittel) & $\begin{array}{l}50 \% \text { Soll } \leq \mathrm{FEV}_{1}<80 \% \text { Soll, }_{\text {FEV }} / \mathrm{VC}<70 \% \\
\text { mit chronischen Symptomen/ohne chronische Symptome } \\
\text { (Husten, Auswurf, Dyspnoe) }\end{array}$ \\
\hline III (schwer) & $\begin{array}{l}30 \% \text { Soll }^{2} \mathrm{FEV}_{1}<50 \% \text { Soll, } \mathrm{FEV}_{1} / \mathrm{VC}<70 \% \\
\text { mit chronischen Symptomen/ohne chronische Symptome } \\
\text { (Husten, Auswurf, Dyspnoe) }\end{array}$ \\
\hline IV (sehr schwer) & $\begin{array}{l}\mathrm{FEV}_{1} \leq 30 \% \text { Soll, } \mathrm{FEV}_{1} / \mathrm{VC}<70 \% \text { oder } \\
\mathrm{FEV}_{1}<50 \% \text { Soll plus chronische respiratorische Insuffizienz }\end{array}$ \\
\hline
\end{tabular}

Tab. 3 Schweregradeinteilung der COPD [5]

\section{Sehr schwere COPD (Schweregrad IV)}

Wenn die $\mathrm{FEV}_{1} \leq 30 \%$ Soll beträgt, liegt ein Schweregrad IV vor. Besteht eine chronische respiratorische Insuffizienz, darf die $\mathrm{FEV}_{1}<50 \%$ Soll betragen, um einen Schweregrad IV zu definieren.
Häufig suchen die Patienten den Arzt anlässlich einer akuten respiratorischen Insuffizienz im Rahmen einer Exazerbation auf. Bei zahlreichen Patienten im Stadium IV treten gehäuft Exazerbationen mit einem potenziell lebensbedrohlichen Verlauf auf. Arterielle Hypoxämie, häufig verbunden mit einer Hyperkapnie, 


\begin{tabular}{|lllll|}
\hline Parameter & \multicolumn{4}{l}{ Punkte auf der BODE Skala } \\
& 0 & 1 & 2 & 3 \\
\hline FEV $_{1}(\%$ Soll $)$ & $\geq 65$ & $50-64$ & $36-49$ & $\geq 35$ \\
\hline 6 Min Gehtest $(\mathrm{m})$ & $>350$ & $250-349$ & $150-249$ & $\leq 149$ \\
\hline MRC Dyspnoe $($ Stufe $)$ & $0-1$ & 2 & 3 & 4 \\
\hline body-mass-index $\left(\mathrm{kg} / \mathrm{m}^{2}\right)$ & $>21$ & $\leq 21$ & & \\
\hline
\end{tabular}

Tab. 4 BODE-Index

Modifizierter MRC Score:

0: keine Atemnot, 1: Atemnot bei schwerer, 2: Atemnot bei leichter Belastung, 3: zu kurzatmig, das Haus zu verlassen, und

4: kurzatmig beim An- und Ausziehen.

\begin{tabular}{|c|c|c|}
\hline Merkmal & Asthma & COPD \\
\hline Alter bei Erstdiagnose & variabel, häufig: Kindheit, Jugend & meist 6. Lebensdekade \\
\hline Tabakrauchen & $\begin{array}{l}\text { kein direkter Kausalzusammen- } \\
\text { hang; Verschlechterung durch } \\
\text { Tabakrauchen möglich }\end{array}$ & direkter Kausalzusammenhang \\
\hline Hauptbeschwerden & anfallsartig auftretende Atemnot & Atemnot bei Belastung \\
\hline Verlauf & variabel, episodisch & progredient \\
\hline Allergie & häufig & selten \\
\hline Obstruktion & variabel & persistierend \\
\hline Reversibilität der Obstruktion & $>20 \% \mathrm{FEV}_{1}$ & $<15 \% \mathrm{FEV}_{1}$ \\
\hline bronchiale Hyperreaktivität & regelhaft vorhanden & möglich \\
\hline Ansprechen auf Glukokortikoide & regelhaft vorhanden & gelegentlich* \\
\hline
\end{tabular}

Tab. 5 Differenzialdiagnose:

Asthma - COPD oder ein Cor pulmonale mit peripheren Ödemen als Zeichen der Rechtsherzinsuffizienz kennzeichnen das Spätstadium der Erkrankung.

Eine neue multidimensionale Schweregradeinteilung, der BODE-Index (B: Body-mass-index, O: obstruction, D: dyspnoea, E: exercise capacity), berücksichtigt Ausmaß der Obstruktion, Atemnot, körperliche Belastbarkeit und body-mass-index [73]. Dieser Index charakterisiert die Beeinträchtigung des Patienten deutlich besser als die $\mathrm{FEV}_{1}$ allein. Die Obstruktion wird anhand der $\mathrm{FEV}_{1}$, die Dyspnoe mittels des (modifizierten) MRC (Medical Research Council) Score, die Belastbarkeit über die 6-MinutenGehstrecke gemessen. Tab. 4 zeigt, wie die Einzelwerte bewertet werden. Durch Addition der Punkte für jeden Parameter ergibt sich der BODE-Score.

Die BODE-Scorebereiche (Quartilen) 0-2, 3-4, 5-6 und 7-10 Punkte korrelierten mit der Gesamtmortalität und mit der COPD bedingten Mortalität in der untersuchten internationalen COPD Patientenpopulation besser als die $\mathrm{FEV}_{1}$ [73].

Anmerkung: In der letzten Fassung der Leitlinie war auch ein Stadium 0 aufgeführt. Das Stadium 0 wurde bei Angabe von chronischem Husten und/oder Auswurf und bei Vorliegen von Risikofaktoren bei gleichzeitig unauffälliger Spirometrie festgestellt. Es wurde angenommen, dass Patienten in diesem Stadium ein gesteigertes Risiko aufweisen, eine manifeste COPD zu entwickeln.

Es gibt zwar Hinweise darauf, dass Männer mit Atembeschwerden, d.h. Dyspnoe, persistierendem Husten und/oder Auswurf ein signifikant gesteigertes Mortalitätsrisiko aufweisen [74]. Andererseits fanden sich in neueren Studien keine Anhaltspunkte dafür, dass Husten und Auswurf (nach Definition der WHO für chronische Bronchitis) allein relevante Risikofaktoren für die Entstehung einer Bronchialobstruktion $[75,76]$ sind. Daher wurde das Stadium 0 in der neuen Fassung der Leitlinie in Übereinstimmung mit dem neuen GOLD-Update gestrichen.

\section{Differenzialdiagnosen}

Die für das Management der COPD wichtigste Differenzialdiagnose ist das Asthma. Charakteristische Merkmale beider Erkrankungen sind in $\bigcirc$ Tab. 5 gegenübergestellt.

Neben Anamnese (Rauchen, atopische Begleiterkrankungen) und Klinik ist das Ausmaß der Reversibilität und der bronchialen Hyperreaktivität ( Tab.5) für die Diagnose entscheidend. Bei Patienten mit widersprüchlichen Untersuchungsergebnissen sollten sowohl Asthma als auch COPD diagnostiziert werden. Die Behandlung sollte dem Schweregrad beider Erkrankungen gerecht werden: Bei COPD sollten zum Beispiel auch bereits bei einer $\mathrm{FEV}_{1}<70 \%$ Soll inhalative Glukokortikoide gegeben werden, wenn gleichzeitig ein Asthma vermutet wird. Andererseits ist bei Asthma mit zusätzlichem COPD-Phänotyp ggf. auch ein langwirksames Anticholinergikum zu verordnen. Maßnahmen der Allergenkarenz und ggf. der antiallergischen Therapie können ebenfalls indiziert sein [78] (Evidenzgrad D, Empfehlungs$\operatorname{grad} \mathrm{A})$.

\section{Weitere differenzialdiagnostische Aspekte}

Alle Erkrankungen mit Bronchialobstruktion (u.a. Bronchiektasie, konstriktive Bronchiolitis, diffuse Lungenparenchymerkrankungen mit Obstruktion wie z.B. Sarkoidose), mit chronischem Husten (u.a. Bronchialkarzinom, Tuberkulose, chronisch persistierender Husten infolge eines gastroösophagealen Refluxes oder einer chronischen Rhinosinusitis) oder Atemnot (u.a. infolge Anämie, Linksherzinsuffizienz, pulmonaler Hypertonie, Übergewicht, Trainingsmangel, Hyperthyreose, metabolischer Azidose) müssen differenzialdiagnostisch oder als Komorbidität abgegrenzt werden. 


$\begin{array}{ll}\text { Prävention } & \begin{array}{l}\text { Medikamentöse Be- } \\ \text { handlung }\end{array} \\ \text { Raucherentwöhnung } & \begin{array}{l}\text { Anticholinergika } \\ \text { Schutzimpfungen }\end{array} \\ \beta_{2} \text {-Sympathomimetika } \\ \text { Arbeitsplatzhygiene } & \text { Theophyllin } \\ & \text { Glukokortikoide } \\ & \text { Mukopharmaka } \\ & \text { Antibiotika }\end{array}$

\section{Nicht medikamentöse}

Behandlung

körperliches Training

Patientenschulung

Physiotherapie

Ernährungsberatung
Apparative/operative

Behandlung

Langzeitsauerstoffthe-

rapie

nichtinvasive Beat-

mung

Emphysemchirurgie

Lungentransplantation
Tab. 6 Therapieoptionen bei COPD

\section{Verlaufsuntersuchungen}

Die COPD ist eine progrediente Erkrankung. Symptome und Kenngrößen der Atemwegsobstruktion sollten bei komplikationslosem Verlauf mindestens $1 \times$ pro Jahr überwacht werden, damit Verschlechterungen bzw. Komplikationen erkannt und behandelt werden können.

Nikotin- und Schadstoffkarenz sind zu prüfen und zu dokumentieren, der weiterhin exponierte Patient ist entsprechend zu beraten. Bei der Kontrolluntersuchung ist die Lungenfunktion zu analysieren und zu dokumentieren (mindestens einmal jährlich). Bei beschleunigtem $\mathrm{FEV}_{1}$-Verlust ( $>30 \mathrm{ml} / \mathrm{Jahr}$ ), der häufig erst auf der Basis von longitudinalen Untersuchungen nach 2-3 Jahren feststellbar ist, sollten die Schadstoffexpositionskarenz und die Exazerbationshäufigkeit gezielt überprüft werden. Auch die spirometrischen (IC) bzw. bodyplethysmographischen Messparameter (RV, etc.), die Überblähung reflektieren, können von Bedeutung für die Verlaufsbeurteilung sein (s.o.).

Die Messung der arteriellen Blutgase sollte bei progredienter Dyspnoe bzw. einer Einschränkung der $\mathrm{FEV}_{1}$ auf weniger als $40 \%$ des Sollwertes oder bei klinischen Zeichen einer respiratorischen Insuffizienz oder einer Rechtsherzinsuffizienz durchgeführt werden. In der Routinediagnostik ist die Messung der Druckverhältnisse im kleinen Kreislauf entbehrlich.

Bei jeder Kontrolluntersuchung sollten die Dosierung und die unerwünschten Wirkungen der Medikamente besprochen, die Inhalationstechnik und der Einsatz nicht medikamentöser Therapiemaßnahmen überprüft und dokumentiert werden.

Die Verlaufskontrolle sollte auch unter dem Aspekt der Kostendämpfung in der Langzeittherapie die Überprüfung der Indikation und der Wirksamkeit eines jeden verordneten Medikamentes unter Einschluss der Verordnung von Sauerstoff beinhalten.

Insbesondere ist die Notwendigkeit einer Intensivierung der Therapie (zum Beispiel Verordnung eines weiteren langwirksamen Bronchodilatators oder von inhalierbaren Glukokortikoiden bei häufigen Exazerbationen) zu evaluieren.

Die Anzahl der zwischen den Verlaufsuntersuchungen aufgetretenen Exazerbationen ist zu notieren. Bei häufigen (>1/Jahr) Exazerbationen sind diagnostische und therapeutische Maßnahmen zur Exazerbationsprophylaxe (Patientenschulung, Impfungen, langwirksame Bronchodilatatoren, inhalative Glukokortikoide bei $\mathrm{FEV}_{1}<50 \%$ Soll, Ausschluss von Bronchiektasen, Suche nach humoraler oder zellulärer Immunabwehrschwäche) zu erwägen. Das Rauchverhalten sollte man analysieren und ggf. erneut intervenieren (Evidenzgrad A ).

Wesentlich ist auch die Dokumentation des Schweregrades der Exazerbation. Zunahme der Sputummenge, Auftreten eines purulenten Sputums und akute Atemnot sollten ebenso registriert werden wie die Notwendigkeit einer Steigerung der Medikation mit Bronchodilatatoren oder Glukokortikoiden sowie der Einsatz von Antibiotika. Schließlich sollten die Hospitalisationen bezüglich Frequenz und Dauer unter Einschluss der Notfallbe- handlungen erfragt werden. Bis zur Hälfte der COPD-Patienten berichten nicht spontan über Exazerbationen [79].

Erforderlich ist die Dokumentation des objektiv festgestellten Körpergewichts und des daraus berechneten body-mass-index (BMI). Bei untergewichtigen COPD Patienten (BMI $<25 \mathrm{~kg} / \mathrm{m}^{2}$ ) sollte eine Ernährungsberatung erfolgen und ggf. Zusatznahrung verordnet werden, da eine Gewichtsnormalisierung die Prognose bessert [80].

Auch die Notwendigkeit einer Rehabilitation ist jährlich zu prüfen.

Darüber hinaus ist die in weniger typischen Fällen einmal gestellte Diagnose COPD - insbesondere in Abgrenzung zum Asthma - bei jeder Konsultation kritisch zu überprüfen. Eine neu aufgetretene oder sich verschlechternde Komorbidität (insbesondere koronare Herzkrankheit, Herzinsuffizienz, Bronchialkarzinom) muss diagnostiziert und ggf. behandelt werden.

Bei Diskrepanz zwischen Atemnot und Lungenfunktion $\left(\mathrm{FEV}_{1}\right.$ $>50 \%$ Soll, aber ausgeprägte Belastungsdyspnoe) ist eine Reevaluation indiziert (Emphysemdiagnostik mit Bodyplethysmographie, Messung der CO Diffusionskapazität, HR-CT etc.).

\section{Therapie}

Der Behandlungsplan kann präventive Maßnahmen, medikamentöse Therapie, Schulung, Physiotherapie, körperliches Training, Ernährungsberatung, apparative Therapieoptionen sowie bei ausgeprägtem Lungenemphysem operative Behandlungsmaßnahmen ( Tab. 6) umfassen. Auch das Management akuter Exazerbationen ist von großer Bedeutung. Zudem stellt die pneumologische Rehabilitation als zeitlich umschriebene komplexe Maßnahme eine wichtige Komponente des Langzeitmanagements der COPD dar.

Einsatz und Auswahl der Therapieoptionen haben die Kooperationsbereitschaft des Patienten zu berücksichtigen.

\section{Prävention}

\section{Leitsätze zur Prävention}

Die Reduktion inhalativer Noxen ist vorrangiges Ziel, um die Entwicklung und die Progression der COPD zu verhindern. Wichtigste Maßnahme ist der Verzicht auf Tabakrauchen (Evidenzgrad A).

Nikotinkarenz vermindert die Exazerbationsfrequenz bei fortgeschrittener COPD $[81,82]$ und die Mortalität [83]. Multimodale Raucherentwöhnungsprogramme mit Verhaltenstherapie, sozialer Unterstützung und einer Pharmakotherapie zur Behandlung der Nikotinabhängigkeit (Bupropion, Nikotinkaugummi, Nikotinpflaster) können die Erfolgsquoten von Entwöhnungsprogrammen steigern (Evidenzgrad A). 
Der Schweregrad einer berufsbedingten COPD kann durch eine Reduktion der inhalativen Noxen vermindert werden

(Evidenzgrad C).

Ziel präventiver Maßnahmen ist die Verhinderung einer weiteren Schädigung der Lunge durch die Beseitigung von Risikofaktoren. Zigarettenrauchen ist in Deutschland der wichtigste Risikofaktor für die COPD (Evidenzgrad A). Die individuelle Empfindlichkeit und damit die pathophysiologischen Konsequenzen sind jedoch unterschiedlich.

Das Fortschreiten der COPD korreliert mit der täglich konsumierten Zigarettenanzahl. Durchschnittlich kommt es bei regelmäßigem Rauchen zu einer gegenüber dem normalen Altersgang beschleunigten $\mathrm{FEV}_{1}$-Abnahme $\mathrm{um} \geq 50 \mathrm{ml} / \mathrm{Jahr}$. Nach Aufgabe des Rauchens kann die weitere jährliche $\mathrm{FEV}_{1}$-Abnahme verlangsamt werden.

\section{Prävention des Tabakrauchens}

Verhaltenspräventive Maßnahmen wie Gesundheitserziehung in der Schule [84] und Aufklärung über die Medien, aber auch gesetzliche Regelungen (z.B. rauchfreier Arbeitsplatz [85], Verbot der Tabakwerbung [86]) sind wesentliche Komponenten einer erfolgreichen Strategie zur Verhinderung des Tabakrauchens (Evidenzgrad A).

\section{Verzicht auf Tabakrauchen}

Der Verzicht auf Tabakrauchen ist die effektivste Einzelmaßnahme zur Reduktion des COPD-Risikos und der Progression der bereits eingetretenen Erkrankung. Selbst eine kurze Beratung (etwa 3 Minuten) führt in 5-10\% der Fälle [87] zur Aufgabe des Nikotinkonsums und sollte als Mindestmaßnahme bei jedem Kontakt mit einem Raucher durchgeführt werden $[87,88]$. Es besteht eine enge Beziehung zwischen der Intensität der Beratung zur Aufgabe des Nikotinkonsums und dem Erfolg [89,90].

\section{Raucherentwöhnung}

Raucherentwöhnungsprogramme zeigen eine Rückfallhäufigkeit von mehr als 80\% nach einem Jahr [91 - 94]. Mithilfe verhaltenstherapeutischer Programme, nikotinhaltiger Pflaster oder Kaugummis lässt sich die Erfolgsquote für motivierte Patienten nach einem Jahr deutlich steigern.

In einer kontrollierten Multicenterstudie ergab die Kombination aus ärztlicher Beratung, Raucherentwöhnung in einer Gruppe unter Einschluss von Entwöhnungstechniken und Nikotinersatztherapie eine Aufgabe des Nikotinkonsums von 35\% der Teilnehmer nach einem Jahr und von $22 \%$ nach 5 Jahren [95]. Wesentliche Punkte [96] für eine erfolgreiche Raucherentwöhnung sind:

Systematische Analyse der Rauchgewohnheiten bei jedem Patienten mit Verdacht auf COPD.

Empfehlung zum Rauchverzicht und dessen Dokumentation bei jedem Arztbesuch.

Kurze Ratschläge bezüglich der Notwendigkeit des Nikotinverzichtes und der Bewältigung von Entwöhnungsproblemen. Hierzu gehört das Erkennen von potenziellen Rückfallsituationen, etwa durch Zeitdruck, schlechte Stimmung oder das Zusammensein mit Rauchern.

Das Führen von Tagebüchern über die Situationen, bei denen Patienten ihre Zigarette anzünden, mit dem Ziel, verhaltenstherapeutische Maßnahmen zum Verzicht auf Nikotin in diesen Situationen einzuleiten.

Weiterleitung von Informationen zur Raucherentwöhnung an andere Betreuer.
Vereinbarung eines Entwöhnungsdatums mit Kontrolluntersuchungen im Verlauf.

Information des Patienten darüber, dass eine langsame Reduktion der Anzahl der Zigaretten in der Regel erfolglos ist, da dieses häufig durch die tiefere Inhalation mit längeren Atemanhaltephasen kompensiert wird.

Berücksichtigung von Nikotinkaugummis und Hautpflastern sowie Bupropion $[97,98]$ zur Behandlung von Entzugssymptomen.

Ermutigung des Patienten zu erneuten Entwöhnungsversuchen auch bei Fehlschlägen, da ein langzeitiger Verzicht von Nikotin häufig erst nach 3-4 Entwöhnungsversuchen erreicht wird.

Diese Maßnahmen sind dem alleinigen ärztlichen Rat bezüglich der Erfolgsrate der Entwöhnung überlegen [95,96,98-100]. Die Pharmakotherapie sollte in der Raucherentwöhnung eingesetzt werden, wenn die nichtmedikamentösen Maßnahmen erfolglos sind. Alle Formen der Nikotinersatztherapie (Nikotinkaugummi, Nikotinpflaster, sublinguale Tablette) führen zu einem höheren Langzeiterfolg [92]. Die Nikotinersatztherapie ist effektiver, wenn sie mit Beratung und verhaltenstherapeutischen Programmen kombiniert wird [101].

Kontraindikationen bezüglich des Einsatzes von Nikotinersatztherapien umfassen die instabile koronare Herzkrankheit, unbehandelte Magengeschwüre, innerhalb der letzten 4 Wochen abgelaufene Herzinfarkte bzw. Schlaganfälle [91]. Die Behandlung mit Nikotinersatzstoffen sollte in der Regel 8 Wochen nicht überschreiten [102]. In Einzelfällen kann ein über 8 Wochen hinausgehender Einsatz der Nikotinersatztherapie zur Vorbeugung von Rückfällen sinnvoll sein. Bei der Auswahl der Applikationsform ist das Nikotinpflaster dem Kaugummi vorzuziehen, da es weniger Training für einen effektiven Einsatz benötigt und mit weniger Complianceproblemen verbunden ist.

Bei Einsatz des Nikotinkaugummis sollte der Patient nach dem Kauen das Gummi gegen die Innenseite der Wange pressen, um eine optimale bukkale Absorption mit verlängerter Freisetzung des Nikotins zu ermöglichen. 15 Minuten vor und nach Einsatz des Kaugummis sollte er weder essen noch Kaffee oder Säfte trinken, um die Absorption des Nikotins nicht zu reduzieren.

Bei starker Abhängigkeit des Rauchers ist zumindest initial das Kaugummi mit einer Dosis von $4 \mathrm{mg}$ Nikotin demjenigen mit einer Dosis von $2 \mathrm{mg}$ vorzuziehen [103].

Nikotinersatztherapie führt in Kombination mit psychosozialer Unterstützung einschließlich adäquater Nachsorge und Rückfallintervention zu einer signifikant höheren Abstinenzrate, die auch nach 5 [95] und 11 [104] Jahren nachweisbar war. Nach dem derzeitigen Kenntnisstand stellt eine individuelle Beratung mit strukturierter Nachsorge und Rückfallmanagement, kombiniert mit der Nikotinersatztherapie, auf Dauer gesehen, die wirksamste Form der Tabakentwöhnung dar [105].

Das Antidepressivum Bupropion steigert über den Effekt von Beratung und den Einsatz einer Nikotinersatztherapie hinaus die Entwöhnungsrate [97,98]. Der Einsatz von Bupropion wird allerdings durch gastrointestinale Nebenwirkungen, gelegentlich aber auch durch Krampfanfälle limitiert. Bei schwerer Leberzirrhose bzw. Neigung zu Krampfanfällen und schwerer instabiler koronarer Herzkrankheit sollte die Substanz nicht eingesetzt werden. Es besteht aber kein Zweifel, dass das Schaden/Nutzenverhältnis bei Einhaltung der Kontraindikationen günstig ausfällt. 
Prophylaxe von arbeitsplatzbezogenen

Schadstoffexpositionen

Berufstätige COPD-Patienten sollten am Arbeitsplatz vor inhalativen Noxen - einschließlich Passivrauchen - geschützt werden $[106,107]$.

\section{Schutzimpfungen}

\section{Influenzaschutzimpfung}

Die Influenzaschutzimpfung sollte jährlich bei allen Patienten mit COPD im Herbst mit der jeweils aktuellen Vakzine durchgeführt werden (Empfehlungsgrad A). Die Influenzaschutzimpfung führt zu einer erheblichen Reduktion der Morbidität, ferner zu einer Abnahme von sekundär auftretenden Pneumonien [108, 109] (Evidenzgrad A).

\section{Influenzaprophylaxe}

COPD-Patienten der Schweregrade III und IV, die Kontakt zu einer an Influenza erkrankten Person - insbesondere während einer Influenzaepidemie - hatten, sollten sofort eine Infektprophylaxe mit Neuraminidasehemmern (Oseltamivir oder Zanamivir) beginnen. Hierzu liegen keine Daten für COPD-Patienten vor, aber die Transmission bei älteren multimorbiden Patienten wird durch diese Maßnahme um 92\% reduziert. Dies gilt auch für geimpfte Personen (91\% Reduktion) [110,111] (Empfehlungs$\operatorname{grad}$ C).

\section{Pneumokokkenschutzimpfung}

Bisher fehlt eine eindeutige Evidenz für den positiven Effekt der Pneumokokkenschutzimpfung bei COPD-Patienten im Hinblick auf die Reduktion von Exazerbationen [112,113]. Wirksam ist die Impfung gegen die bakteriämische Form der Pneumokokkenpneumonie mit hoher Mortalität [114]. Allerdings ist eine erhöhte Inzidenz von Pneumokokkenpneumonien bei Patienten mit COPD in dieser Population nicht gesichert [115].

Bei älteren, chronisch kranken Patienten mit und ohne COPD überwiegen jedoch die potenziellen Vorteile gegenüber den Risiken der Pneumokokkenschutzimpfung [116].

Deshalb kann die Pneumokokkenschutzimpfung für alle COPDPatienten empfohlen werden (Evidenzgrad D). Eine Wiederholungsimpfung sollte im Abstand von 5-6 Jahren nach der Erstimpfung durchgeführt werden [117].

\section{Langzeitbehandlung der stabilen COPD}

\section{Leitsätze}

Die Langzeittherapie der stabilen COPD ist durch eine schrittweise Steigerung der Therapiemaßnahmen in Abhängigkeit vom Schweregrad der Erkrankung gekennzeichnet. Sie umfasst regelhaft sowohl medikamentöse als auch nichtmedikamentöse Therapieverfahren.

Mit keinem der vorhandenen medikamentösen Therapieansätze lässt sich nach bisheriger Datenlage die Progression der Beeinträchtigung der Lungenfunktion beeinflussen (Evidenzgrad A). Die Pharmakotherapie ermöglicht eine Linderung der Beschwerden, eine Besserung von körperlicher Leistungsfähigkeit und Lebensqualität und/oder eine Verminderung der Exazerbationsfrequenz.

Bronchodilatatoren (Anticholinergika, $\beta_{2}$-Sympathomimetika und Theophyllin) sind die Basismedikamente zur Linde- rung der Beschwerden bei COPD (Evidenzgrad A). Die Wahl zwischen Anticholinergika und $\beta_{2}$-Sympathomimetika hängt vom individuellen Ansprechen des Patienten bezüglich der Effekte und der unerwünschten Wirkungen ab (Evidenzgrad A). Langwirksame Bronchodilatatoren ( $\beta_{2}$-Sympathomimetika und das Anticholinergikum Tiotropium) sind effektiver und einfacher anzuwenden als kurzwirksame, aber auch teurer (Evidenzgrad A). Theophyllin kann in der Langzeittherapie der COPD effektiv sein, sollte aber wegen zahlreicher Interaktionen und der relativ geringen therapeutischen Breite als Bronchodilatator der 3. Wahl eingesetzt werden. Eine Dauerbehandlung mit inhalativen Glukokortikoiden sollte bei COPD-Patienten mit einer postbronchodilatatorischen $\mathrm{FEV}_{1}<50 \%$ Soll und mindestens einer den Einsatz von systemischen Steroiden und/oder Antibiotika notwendig machenden COPD-Exazerbation im letzten Jahr zum Einsatz kommen (Evidenzgrad A, Empfehlungsgrad A). Eine Dauerbehandlung mit systemischen Glukokortikoiden sollte wegen der häufigen unerwünschten Effekte vermieden werden (Evidenzgrad A).

Patienten mit COPD profitieren von körperlichem Training bezüglich Belastbarkeit und Linderung von Dyspnoe und Ermüdbarkeit (Evidenzgrad A, Empfehlungsgrad A). Die Langzeitsauerstofftherapie über 16-24 Stunden am Tag bei Patienten mit chronischer respiratorischer Insuffizienz $\left(\mathrm{pO}_{2}<55 \mathrm{~mm} \mathrm{Hg}\right)$ verbessert die Prognose der Patienten (Evidenzgrad A).

Die Patientenschulung ist ein wichtiges Therapieelement für alle Schweregrade der Erkrankung, da sie zu einer Steigerung der Effizienz des Managements wesentlich beiträgt (Evidenzgrad B, Empfehlungsgrad A).

Die pneumologische Rehabilitation als zeitlich umschriebene komplexe Maßnahme (medikamentöse und nichtmedikamentöse Therapieverfahren) stellt eine wichtige und effektive Komponente des Langzeitmanagements der COPD dar (Evidenzgrad A, Empfehlungsgrad A).

Eine effiziente Langzeitbetreuung bedarf einer eng verzahnten Versorgung durch Hausarzt, Facharzt, Akutkrankenhaus/ Fachklinik sowie stationärer und ambulanter Rehabilitation (Evidenzgrad D).

Ziele der Pharmakotherapie sind die Besserung der Symptome, eine Zunahme der körperlichen Leistungsfähigkeit, eine Steigerung der Lebensqualität sowie die Prävention von Exazerbationen.

Eine Normalisierung der Lungenfunktion ist bei Patienten mit COPD nicht zu erwarten. Falls sich die Lungenfunktion unter einer Therapie normalisiert, muss die Diagnose COPD revidiert werden. Meist handelt es sich in solchen Fällen um ein Asthma. Weder für Bronchodilatatoren noch für inhalative Glukokortikoide ist bislang eine Besserung der Prognose nachgewiesen [95,118-122].

Husten und Auswurf bessern sich nach Ausschalten exogener Noxen, insbesondere nach Aufgabe des Nikotinkonsums. Die Dyspnoe von Patienten mit COPD wird oft durch $\beta_{2}$-Sympathomimetika, Anticholinergika und Theophyllin günstig beeinflusst. Wahl und Dosierung sollen individuell unter Berücksichtigung des Schweregrades der Erkrankung sowie von Wirksamkeit und Verträglichkeit erfolgen.

Bei ungenügendem Effekt kann eine Kombination von inhalierbaren langwirksamen Bronchodilatatoren (Tiotropium und ein langwirksames $\beta_{2}$-Sympathomimetikum) zum Einsatz kommen. Bei einer $\mathrm{FEV}_{1}<50 \%$ des Solls (postbronchodilatatorisch) sowie 


\begin{tabular}{|c|c|c|c|c|}
\hline Schweregrad & I: leicht & II: mittel & III: schwer & IV: sehr schwer \\
\hline \multirow[t]{5}{*}{ Charakteristika } & $\begin{array}{l}\text { - } \mathrm{FEV}_{1} / \mathrm{VC}<70 \% \\
\cdot \mathrm{FEV}_{1} \geq 80 \% \\
\cdot \text { mit/ohne } \\
\text { Symptomatik }\end{array}$ & \begin{tabular}{|l|}
$\cdot-\mathrm{FEV}_{1} / \mathrm{VC}<70 \%$ \\
$\cdot 50 \% \leq \mathrm{FEV}_{1}<80 \%$ \\
$\cdot$ mit/ohne \\
Symptomatik
\end{tabular} & $\begin{array}{l}\cdot \mathrm{FEV}_{1} / \mathrm{VC}<70 \% \\
\cdot 30 \%<\mathrm{FEV}_{1}<50 \% \\
\cdot \text { mit/ohne } \\
\text { Symptomatik }\end{array}$ & $\begin{array}{l}\text { - } \mathrm{FEV}_{1} / \mathrm{VC}<70 \% \\
\text { - } \mathrm{FEV}_{1 \leq 30 \% \text { oder }} \\
\text { - FEV1 } 1<50 \% \text { und chronische } \\
\text { respiratorische Insuffizienz, } \\
\text { Zeichen der Rechtsherz- } \\
\text { insuffizienz }\end{array}$ \\
\hline & \multicolumn{4}{|c|}{$\begin{array}{l}\text { Vermeidung von Risikofaktoren, Grippe- und Pneumokokken-Schutzimpfung. } \\
\text { Zusätzlich bei Bedarf kurzwirksamer Bronchodilatator. }\end{array}$} \\
\hline & & \multicolumn{3}{|c|}{$\begin{array}{l}\text { Zusätzlich Dauertherapie mit einem oder mehreren langwirksamen } \\
\text { Bronchodilatatoren, Rehabilitation. }\end{array}$} \\
\hline & & & \multicolumn{2}{|c|}{$\begin{array}{l}\text { Zusätzlich inhalative Glukokortikoide bei } \\
\text { wiederkehrenden Exazerbationen. }\end{array}$} \\
\hline & & & & $\begin{array}{l}\text { Zusätzlich Langzeitsauerstoff- } \\
\text { therapie bei respiratorischer } \\
\text { Insuffizienz. } \\
\text { Prüfen, ob chirurgische } \\
\text { Behandlung angezeigt ist. }\end{array}$ \\
\hline
\end{tabular}

Abb. 3 Stufenplan für die Prophylaxe und Langzeittherapie der COPD.

Tab. 7 Einzeldosis und Wirkdauer der wesentlichen Bronchodilatatoren in der Langzeittherapie der COPD

\begin{tabular}{|c|c|c|c|c|}
\hline Medikament & \multicolumn{4}{|c|}{ Pulverinhalator $(\mu g)$} \\
\hline \multicolumn{5}{|l|}{ Anticholinergika } \\
\hline Ipratropiumbromid & $20-40 / 200$ & $0,25-0,5$ & - & $6-8$ \\
\hline Tiotropiumbromid & 18 & - & - & 24 \\
\hline \multicolumn{5}{|l|}{ beta $_{2}$-Sympathomimetika } \\
\hline Fenoterol & $100-200$ & $0,2-0,4$ & - & $4-6$ \\
\hline Salbutamol & $100-200$ & $1,25-2,5$ & $4-8$ & $4-6 * * *$ \\
\hline Terbutalin & 500 & $2,5-10$ & $2,5-7,5$ & $4-6 * * *$ \\
\hline Bambuterol & - & - & 10 & 24 \\
\hline Formoterol & $6-24$ & & - & 12 \\
\hline Salmeterol & $50-100$ & & - & 12 \\
\hline \multicolumn{5}{|l|}{ Methylxanthine** } \\
\hline Theophyllin (retard) & - & - & $100-500$ & variabel $(\leq 24)$ \\
\hline \multicolumn{5}{|l|}{ Kombinationspräparate } \\
\hline Fenoterol + Ipratropiumbromid & $50 / 20-100 / 40$ & $0,05 / 0,025-0,1 / 0,05$ & & $6-8$ \\
\hline
\end{tabular}

mindestens einer den Einsatz von systemischen Steroiden und/ oder Antibiotika erforderlich machenden Exazerbation im letzten Jahr ist die Therapie mit inhalativen Glukokortikoiden indiziert. Der Stufenplan für die Langzeittherapie ist in $\mathbf{A b b . ~} \mathbf{3}$ dargestellt.

Wirksamkeitskriterien zur Beurteilung des Therapieerfolges sind die Beeinflussung des Befindens (Gesundheitsstatus, Lebensqualität), der Symptome (Atemnot in Ruhe und/oder bei Belastung, Husten, Auswurf), der körperlichen Belastbarkeit sowie von Kenngrößen der Lungenfunktion ( $\mathrm{FEV}_{1}$, Resistance, intrathorakales Gasvolumen, Residualvolumen, arterielle Blutgase).

\section{Bronchodilatatoren}

Bronchodilatatoren ( Tab. 7) stellen die Basistherapie des symptomatischen Patienten mit COPD dar. Durch Reduktion des Bronchialmuskeltonus und damit des Atemwegswiderstandes und durch Abnahme der Lungenüberblähung führen sie zu einer Symptomlinderung. Die inhalative Applikation ist der oralen Applikation vorzuziehen, da mit geringeren Dosen gleiche Effekte erreicht werden und damit weniger unerwünschte Wir- kungen in Kauf genommen werden müssen. Bei allen inhalativ verabreichten Bronchodilatatoren muss die Inhalationstechnik mit dem Patienten eingeübt und im Therapieverlauf überprüft werden.

Bei fehlendem Ansprechen auf Bronchodilatatoren sollte die $\mathrm{Zu}-$ verlässigkeit der Medikamenteneinnahme unter Einschluss der Inhalationstechnik geprüft werden. Bei unzureichender Inhalationstechnik auch nach einem entsprechenden Training ist die Auswahl eines anderen Applikationssystems, gegebenenfalls der Einsatz einer Inhalationshilfe sowie die Anwendung eines Verneblers zu prüfen [123]. Für Patienten, die trotz entsprechender Anweisung und Anwendung von Inhalationshilfen eine effektive Inhalationstechnik nicht erlernen können, stehen Vernebler zur Verfügung, über die $\beta_{2}$-Sympathomimetika und/oder Anticholinergika appliziert werden können. Gelingt eine effektive Inhalation auch mithilfe von Verneblern nicht, können orale $\beta_{2}$-Sympathomimetika in Retardform eingesetzt werden. Sollte die Medikation nicht zu einer Besserung der Symptomatik und/ oder der Lungenfunktion führen, ist die Umsetzung auf einen an- 
deren Bronchodilatator zu versuchen. Bei regelmäßiger Anwendung von Bronchodilatatoren sind kurzwirksame Substanzen billiger, aber wegen der erforderlichen 3-4-maligen Gabe pro Tag bezüglich der Therapietreue ungünstiger als langwirksame Substanzen mit 1-2-maliger täglicher Einnahme. Daneben ist die Therapie mit langwirksamen Bronchodilatatoren effektiver $[52,124,125]$.

\section{Anticholinergika}

Anticholinergika (Ipratropiumbromid, Tiotropiumbromid) erweitern die Bronchien, vermindern die Schleimsekretion, verringern das Dyspnoe-Empfinden, bessern die körperliche Leistungsfähigkeit [44] und reduzieren Exazerbationen [126,127] bei Patienten mit COPD. Die volle Wirkung des kurzwirksamen Anticholinergikums Ipratropiumbromid tritt erst 20-30 Minuten nach Inhalation ein, hält aber länger an als die der kurzwirksamen $\beta_{2}$-Sympathomimetika, weshalb bei regelmäßiger Medikation eine 3-4-malige Anwendung pro Tag empfohlen wird.

Die meisten klinischen Untersuchungen zeigen bei Vergleich der kurzwirksamen $\beta_{2}$-Sympathomimetika mit den kurzwirksamen Anticholinergika eine ähnlich gute Wirkung $[128,129]$, nach einigen Studien sogar einen besseren Langzeiteffekt der Anticholinergika $[130,131]$. Die einzige klinisch relevante unerwünschte Wirkung ist Mundtrockenheit. Selten können Harnverhalt, Verschlechterung des Augendruckes bei Engwinkelglaukom und Herzrhythmusstörungen auftreten.

Tiotropiumbromid, ein langwirksames Anticholinergikum, führt praktisch zu einer 24 Stunden anhaltenden Bronchodilatation [49,132 - 134] und wird deshalb einmal täglich appliziert. Langzeitstudien mit Tiotropiumbromid zeigen gegenüber Plazebo eine signifikant bessere Bronchodilatation, eine Reduktion von Überblähung [135], Atemnot, Exazerbationen [136] und Hospitalisationen. In Subgruppenanalysen der Studien von Niewoehner u. Mitarb. und Dusser u. Mitarb. zeigte sich, dass die Exazerbationsfrequenz durch Tiotropium sowohl bei den Patienten mit als auch bei denen ohne gleichzeitige Behandlung mit inhalierbaren Steroiden gesenkt wurde [136,137]. Weiter kann Tiotropium die Belastbarkeit steigern $[135,138]$ und eine Besserung der Lebensqualität [51] bewirken.

In 2 Vergleichsstudien zwischen Tiotropium und Salmeterol, die zusammen ausgewertet wurden, hatte Tiotropium ausgeprägtere Effekte als Salmeterol [139].

Im Vergleich zu Ipratropiumbromid $(4 \times 40 \mu \mathrm{g} /$ Tag $)$ erweist sich Tiotropiumbromid $(1 \times 18 \mu \mathrm{g} / \mathrm{Tag})$ bezüglich der Beeinflussung o.a. Wirksamkeitskriterien als überlegen [52] (Evidenzgrad B).

\section{$\beta_{2}$-Sympathomimetika}

Die Inhalation rasch wirksamer $\beta_{2}$-Sympathomimetika mittels Dosier-Aerosol oder Pulver ist die Basistherapie bei akuter Atemnot. Dazu gehören die kurzwirksamen $\beta_{2}$-Sympathomimetika mit den Substanzen Fenoterol, Salbutamol und Terbutalin. Daneben ist auch das rasch und lang wirksame $\beta_{2}$-Sympathomimetikum Formoterol als Bedarfsmedikament für die Behandlung der COPD zugelassen [140].

Trotz gewisser Bedenken bezüglich der Effekte einer Dauermedikation mit kurzwirksamen $\beta_{2}$-Sympathomimetika spricht die gegenwärtig vorliegende Studienlage nicht gegen einen derartigen Einsatz bei COPD [122,141], da bisher kaum Hinweise auf das Auftreten einer klinisch relevanten Tachyphylaxie dokumentiert sind.

Langwirksame $\beta_{2}$-Sympathomimetika (Salmeterol, Formoterol) mit einer Wirkdauer von etwa 12 Stunden führen zu einer Re- duktion der Atemnot am Tage und in der Nacht [142-144], zu einer Besserung der Lungenfunktion [145] inklusive einer Abnahme der Überblähung [146], einer Verbesserung der Lebensqualität [147] und einer Reduktion von Exazerbationen [148]. In einer Studie mit Salmeterol konnte eine Steigerung der Belastbarkeit [146] nachgewiesen werden.

An unerwünschten Wirkungen von $\beta_{2}$-Sympathomimetika sind vor allem Herzrhythmusstörungen (Vorhofflattern, ventrikuläre Extrasystolie, supraventrikuläre Tachykardien), in seltenen Fällen Angina pectoris sowie Palpitationen zu nennen, die insbesondere bei Vorliegen einer chronischen Hypoxämie [149] auftreten können. Eine meist leicht beherrschbare Hypokaliämie kann ebenfalls vorkommen.

Insbesondere bei Patienten mit Herzinsuffizienz und koronarer Herzkrankheit und Neigung zu Herzrhythmusstörungen sollte der Einsatz von $\beta_{2}$-Sympathomimetika unter sorgfältiger Kontrolle bzgl. Arrhythmien und koronaren Ischämiezeichen erfolgen.

Es ist möglich, dass zwischen den beiden am Markt verfügbaren langwirksamen $\beta_{2}$-Sympathomimetika bzw. zwischen diesen und Tiotropium Unterschiede bzgl. der Effekte auf Frequenz und Schweregrad von COPD-Exazerbationen bestehen. Aus Sicht der Autoren der Leitlinie erlaubt die gegenwärtig vorliegende Studienlage aber keine evidenzbasierte Aussage hierzu. Die Gründe hierfür sind:

Die in den einzelnen Studien angewandten Exazerbationsdefinitionen sind unterschiedlich. Eine Exazerbationsdefinition, die sich auf eingeleitete ärztliche Maßnahmen stützt, ist nicht vergleichbar mit einer, die auf Symptomen/Befunden aufbaut.

In manchen Studien wurden nur die Effekte der zu testenden Substanz geprüft, andere ließen zu, dass die zu prüfende Substanz zu einer bestehenden Medikation zusätzlich gegeben wurde.

Relevante Direktvergleiche von $\beta_{2}$-Sympathomimetika und Anticholinergika mit ausreichender Laufzeit (mind. 1 Jahr) wurden bisher nicht publiziert.

\section{Theophyllin}

Theophyllin ist ein schwächerer Bronchodilatator als Anticholinergika oder $\beta_{2}$-Sympathomimetika $[150,151]$. Bei Dosierungen im oberen therapeutischen Bereich (Serumspiegel: 10 - $15 \mathrm{mg} / \mathrm{l}$ ) werden auch positive Effekte auf die Symptomatik und die Belastbarkeit der COPD-Patienten berichtet [152-155]. Neben der Bronchodilatation werden bei Patienten mit COPD eine Steigerung der Atemmuskelkraft [155] sowie eine Zunahme der Ejektionsfraktion des rechten Ventrikels beobachtet, deren klinische Bedeutung für die Langzeitbehandlung allerdings kontrovers diskutiert wird. Hinsichtlich einer Senkung der Exazerbationsfrequenz liegen für Theophyllin keine relevanten Daten vor. Für die Langzeittherapie sind ausschließlich Theophyllinpräparate mit verzögerter Wirkstofffreisetzung (Retardpräparate) geeignet. Limitierend für die Anwendung sind die Häufigkeit unerwünschter Effekte [156], die geringe therapeutische Breite und die Abhängigkeit der Theophyllinclearance von zahlreichen Einflussgrößen. So steigern das Tabakrauchen, proteinreiche Kost und einige Medikamente (z. B. Rifampicin) die Theophyllinclearance, während andere, z.B. einige Antibiotika (Ciprofloxacin, Erythromycin, Clarithromycin, Roxithromycin), Cimetidin, Allopurinol, aber auch Leberkrankheiten, Herzinsuffizienz, Pneumonien und Virusinfekte zu einer Reduktion der Theophyllinclearance führen. Wiederholte Blutspiegelbestimmungen zur Über- 
prüfung der gewählten Dosierung und der Patientencompliance sind anzuraten. Die Blutentnahme sollte 12 Stunden nach der letzten Einnahme erfolgen. Ein Blutspiegelbereich zwischen 10 und $15 \mathrm{mg} / \mathrm{l}$ sollte angestrebt, ein Blutspiegel von $15 \mathrm{mg} / \mathrm{l}$ nicht überschritten werden. Wesentliche unerwünschte Effekte der Theophyllintherapie sind Übelkeit, Erbrechen, abdominelle Schmerzen, Schlafstörungen, Muskelkrämpfe, Hypokaliämie und tachykarde Herzrhythmusstörungen, die gelegentlich schon bei Serumkonzentrationen im therapeutischen Bereich auftreten können.

Etwa die Hälfte der Patienten mit COPD profitiert von einer Theophyllintherapie [157]. Die geeignete Methode, diese „Responder“ zu identifizieren, ist der Auslassversuch über 3 Tage nach vorheriger Theophyllinbehandlung. In einer stabilen Krankheitsphase wird Theophyllin abgesetzt. Nur dann, wenn im Verlauf der folgenden 1-3 Tage die Dyspnoe zunimmt bzw. sich die Funktionswerte verschlechtern, ist eine Langzeittherapie gerechtfertigt. Andernfalls kann auf die ständige Theophyllingabe verzichtet werden.

\section{Kombinationen von Bronchodilatatoren}

Die Kombination aus kurzwirksamen $\beta_{2}$-Sympathomimetikum und Anticholinergikum $[130,158]$ bzw. Theophyllin [159] hat gegenüber den Einzelsubstanzen einen additiven bronchodilatatorischen Effekt (Evidenzgrad $A$ ). Ein äquivalenter therapeutischer Effekt kann auch durch Dosissteigerung eines Bronchodilatators erreicht werden, wenn unerwünschte Wirkungen diese Dosiserhöhung nicht limitieren. Kontrollierte Studien zur Bewertung dieser beiden therapeutischen Alternativen liegen nicht vor.

Bei älteren Patienten mit unerwünschten Effekten nach Inhalation von $\beta_{2}$-Sympathomimetika (Tremor, Tachykardie) kann durch Reduktion des $\beta_{2}$-Sympathomimetikums bei zusätzlicher Gabe eines Anticholinergikums eine nebenwirkungsärmere Behandlung erreicht werden. Außerdem lässt sich durch Kombination eines Anticholinergikums mit einem kurzwirksamen $\beta_{2}$-Sympathomimetikum gegenüber dessen alleiniger Gabe die Exazerbationsrate senken [160]. Die Kombination aus $\beta_{2}$-Sympathomimetikum mit einem Anticholinergikum und/oder Theophyllin kann zusätzlich zu Besserungen der Lungenfunktion [130,158, 161] und des Gesundheitsstatus [127,162] beitragen. Gewöhnlich erhöht die Anzahl der Medikamente allerdings die Therapiekosten und verschlechtert die Therapieadhärenz, wenn es sich nicht um ein Kombinationspräparat handelt.

Die Kombination von Tiotropium und Formoterol erwies sich hinsichtlich der Verbesserung der Lungenfunktion (FEV,$F V C)$ als wirksamer als die Einzelsubstanzen [163] (Evidenzgrad B).

\section{Inhalation mit Verneblern}

Die subjektiv empfundene Zunahme der Wirksamkeit bei akuter Atemnot [164] von $\beta_{2}$-Sympathomimetika oder Anticholinergika beim Einsatz über Vernebler bzw. mittels intermittierender Überdruckinhalation (IPPB) liegt an einer höheren Deposition der Wirkstoffe in den unteren Atemwegen, vor allem aber daran, dass die Wirkstoffe in Inhalationslösungen meist höher dosiert sind als in Dosieraerosolen oder Pulverinhalatoren. Die Notwendigkeit der Koordination oder der Auslösung eines Pulverinhalators durch den stark dyspnoeischen Patienten entfällt. Vorteile einer dauerhaften Therapie mit Verneblern gegenüber DosierAerosolen oder Pulverinhalatoren sind nicht belegt.

Als Nachteile der Inhalationstherapie mit Verneblern sind die Notwendigkeit der Wartung und Desinfektion des Inhaliergerätes und die deutlich höheren Therapiekosten zu nennen. Im All- gemeinen benötigen Patienten in der stabilen Phase der Erkrankung keine Vernebler zur Dauermedikation, es sei denn, dass die Inhalationstechnik nicht adäquat erlernt werden kann.

\section{Glukokortikoide}

Glukokortikoide werden in der Behandlung der COPD in großem Umfang eingesetzt.

Es gibt Anhaltspunkte dafür, dass die Langzeitanwendung von inhalierbaren Steroiden zu einer Reduktion der Atemwegsentzündung führt [165]. Die Lungenfunktion wird im Gegensatz zum Asthma allenfalls geringfügig gebessert [166,167].

\section{Inhalative Glukokortikoide}

Inhalative Glukokortikoide werden bei Patienten mit obstruktiven Lungenkrankheiten häufig ohne eine klare Differenzierung zwischen Asthma und COPD eingesetzt. Findet sich bei Patienten mit COPD eine asthmatische Komponente, etwa mit Vorliegen einer bronchialen Hyperreaktivität und einem Anstieg der FEV um mehr als $15 \%$ bzw. $200 \mathrm{ml}$ gegenüber dem Ausgangswert nach mindestens 2-4-wöchiger Applikation von Glukokortikoiden (Reversibilitätstest, siehe Diagnostik), sollten diese Patienten wie Asthmatiker behandelt werden.

Bei Patienten mit COPD ohne positiven Reversibilitätstest nach Inhalation von Bronchodilatatoren sind nach Langzeituntersuchungen [118-121] keine erkennbaren positiven Effekte inhalativer Glukokortikoide auf die jährliche Abnahme des FEV $_{1}$ vorhanden (Evidenzgrad A).

Bei symptomatischen COPD-Patienten der Schweregrade III und IV ist eine Dauertherapie mit inhalativen Glukokortikoiden indiziert, wenn nicht nur ein $\mathrm{FEV}_{1}<50 \%$ Soll vorliegt, sondern zusätzlich mindestens eine den Einsatz von systemischen Steroiden und/oder Antibiotika notwendig machende Exazerbation im Vorjahr aufgetreten ist. Für COPD-Patienten mit dieser Konstellation konnte eine Reduktion der Exazerbationsfrequenz durch den Einsatz inhalativer Steroide gezeigt werden [54,120,168,169] (Evidenzgrad A).

In mehreren Studien haben sich Anhaltspunkte dafür ergeben, dass das Absetzen von inhalierbaren Glukokortikoiden negative Auswirkungen auf Lungenfunktion und Symptome haben kann [170]. Weiter ist eine Zunahme der Exazerbationshäufigkeit [171] möglich. Vor diesem Hintergrund müssen Patienten, bei denen inhalierbare Steroide abgesetzt werden, sorgfältig kontrolliert werden. Es ist festzuhalten, dass es für derartige StepDown-Maßnahmen in keiner der internationalen Leitlinien eine Empfehlung gibt.

Klare Dosis-Wirkungsbeziehungen sind für inhalative Glukokortikoide bei COPD nicht bekannt. Bei Langzeitanwendung höherer Dosierungen ist gehäuft mit Mundsoor zu rechnen und auch eine Abnahme der Knochendichte möglich [121]. Darüber hinaus besteht offensichtlich zumindest bei den für die Therapie der COPD in Deutschland zugelassenen hohen Dosen an inhalativen Glukokortikoiden ein gesteigertes Risiko für die Entwicklung einer Pneumonie [172] (Evidenzgrad A).

\section{Systemische Glukokortikoide}

Eine Langzeittherapie mit oralen Glukokortikoiden wird bei der COPD nicht empfohlen [167,173] (Evidenzgrad A).

Wegen der potenziell schwerwiegenden unerwünschten Wirkungen darf die Indikation nur dann gestellt werden, wenn positive Effekte eindeutig dokumentiert sind. Es handelt sich in diesen Fällen um Patienten mit einer Asthma-Komponente. Besonders zu beachten ist die Steroidmyopathie, die schon bei relativ 
Tab. 8 Effekte von häufig angewendeten Medikamenten auf wichtige klinische Parameter der COPD

\begin{tabular}{|c|c|c|c|c|c|c|c|}
\hline & $\mathrm{FEV}_{1}$ & $\begin{array}{l}\text { Lungen- } \\
\text { volumen }\end{array}$ & Dyspnoe & HRQol & $\begin{array}{l}\text { Exazerba- } \\
\text { tionen }\end{array}$ & $\begin{array}{l}\text { Belast- } \\
\text { barkeit }\end{array}$ & $\begin{array}{l}\text { Neben- } \\
\text { wirkungen }\end{array}$ \\
\hline $\begin{array}{l}\text { kurzwirksame } \\
\beta_{2} \text {-Sympathomimetika }\end{array}$ & Ja (A) & Ja (B) & $\mathrm{Ja}(\mathrm{A})$ & N.B. & N.B. & $\mathrm{Ja}(\mathrm{B})$ & einzelne \\
\hline $\begin{array}{l}\text { langwirksame } \\
\beta_{2} \text {-Sympathomimetika }\end{array}$ & $\mathrm{Ja}(\mathrm{A})$ & Ja (A) & $\mathrm{Ja}(\mathrm{A})$ & $\mathrm{Ja}(\mathrm{A})$ & $\mathrm{Ja}(\mathrm{A})$ & $\mathrm{Ja}(\mathrm{B})$ & minimal \\
\hline Ipratropium & $\mathrm{Ja}(\mathrm{A})$ & $\mathrm{Ja}(\mathrm{B})$ & $\mathrm{Ja}(\mathrm{A})$ & Nein (B) & $\mathrm{Ja}(\mathrm{B})$ & $\mathrm{Ja}(\mathrm{B})$ & einzelne \\
\hline Tiotropium & $\mathrm{Ja}(\mathrm{A})$ & $\mathrm{Ja}(\mathrm{A})$ & $\mathrm{Ja}(\mathrm{A})$ & $\mathrm{Ja}(\mathrm{A})$ & $\mathrm{Ja}(\mathrm{A})$ & $\mathrm{Ja}(\mathrm{A})$ & minimal \\
\hline inhalierbare Glukokortikoide* & $\mathrm{Ja}(\mathrm{A})^{* *}$ & N.B. & $\mathrm{Ja}(\mathrm{B})$ & $\mathrm{Ja}(\mathrm{A})$ & $\mathrm{Ja}(\mathrm{A})$ & N.B. & einzelne \\
\hline Kombinationen & $\mathrm{Ja}(\mathrm{A})$ & N.B. & $\mathrm{Ja}(\mathrm{A})$ & $\mathrm{Ja}(\mathrm{A})$ & $\mathrm{Ja}(\mathrm{A})$ & N.B. & einzelne \\
\hline Theophyllin & Ja (A) & $\mathrm{Ja}(\mathrm{B})$ & $\mathrm{Ja}(\mathrm{A})$ & $\mathrm{Ja}(\mathrm{B})$ & N.B. & $\mathrm{Ja}(\mathrm{B})$ & bedeutend \\
\hline
\end{tabular}

* als Zusatztherapie

** Die FEV $_{1}$ steigt zu Beginn der Therapie signifikant an. Der Verlauf der FEV 1 über die Zeit wird nicht beeinflusst.

In Klammern ist das Evidenzniveau für den jeweiligen Parameter angegeben.

N.B. $=$ bislang nicht bestimmt.

Mit „Kombinationen“ sind feste Kombinationen von inhalierbaren Steroiden und langwirkenden $\beta_{2}$-Sympathomimetika gemeint, modifiziert nach [3].

geringen Tagesdosen (<10 mg Prednisolonäquivalent) auftreten und die Entwicklung einer respiratorischen Insuffizienz begünstigen kann $[173,174]$. Dosisabhängig ist mit einer erhöhten Letalität zu rechnen [175].

\section{Kombinationen aus langwirksamen}

\section{$\beta_{2}$-Sympathomimetika und Glukokortikoiden}

Gegenwärtig liegen die Ergebnisse von 6 randomisierten, doppelblinden, plazebokontrollierten Studien als Vollpublikationen vor, die die Effekte der Kombinationspräparate aus Salmeterol/ Fluticason und Formoterol/Budesonid evaluiert haben [54,168, 169,176-178].

Die Kombinationstherapie ist gegenüber Plazebo hinsichtlich Exazerbationsraten, Lungenfunktion und Lebensqualität überlegen (Evidenzgrad A). In einer großen Studie [54] hat die Kombination Fluticason/Salmeterol die Exazerbationsrate im Vergleich zu den Einzelsubstanzen nicht signifikant gesenkt. In zwei Studien war die Kombination Budesonid/Formoterol bzgl. der Senkung der Exazerbationsrate signifikant besser als Formoterol $[168,169]$.

Hinsichtlich der Lebensqualität sind die Ergebnisse der Kombinationspräparate in den genannten Studien im Vergleich mit den Einzelsubstanzen uneinheitlich. Bezüglich der Symptome und der Häufigkeit der Anwendung von Bedarfsmedikation waren beide Kombinationen den Einzelsubstanzen überlegen $[54,168,169]$ (Evidenzgrad A). Die Behandlung mit beiden Kombinationen führt zu einer signifikanten Verbesserung der Lungenfunktion im Vergleich mit der alleinigen Gabe des inhalierbaren Glukokortikosteroids. Fluticason/Salmeterol war hinsichtlich der erzielten Lungenfunktionsveränderungen Salmeterol überlegen, während Budesonid/Formoterol keine signifikanten Unterschiede zur Behandlung mit Formoterol zeigte. Mögliche Auswirkungen der Kombinationspräparate auf die Therapieadhärenz und damit den Langzeiterfolg sind bislang nicht untersucht.

- Tab. 8 fasst die Effekte von häufig für die Behandlung der COPD angewendeten Medikamenten zusammen. Die angegebenen Evidenzniveaus beziehen sich auf den Vergleich zu Plazebo. Anmerkung: Abweichend von den ATS/ERS-Empfehlungen scheint unter Berücksichtigung von neuen Studien, die bei o.g. Klassifizierung noch nicht vorlagen, $[135,138,179]$ ein Evidenzgrad A für die Effekte von Tiotropium auf die Belastbarkeit gerechtfertigt. Im Gegensatz dazu ist der Evidenzgrad B für die Besserung der Belastbarkeit durch langwirksame $\beta_{2}$-Sympatho- mimetika weiterhin adäquat, da zwei Studien für Salmeterol $[135,180]$ und eine Studie für Formoterol [181] signifikante Effekte zeigten, eine Reihe von anderen Untersuchungen [182 -186] aber keine signifikante Besserung der Belastbarkeit ergaben.

\section{Mukopharmaka}

Die Indikation zum Einsatz von Mukopharmaka zur besseren Sekretelimination sollte kritisch gestellt werden und sich an dem subjektiven Therapieerfolg orientieren. N-Acetylcystein, Ambroxol, Myrthol und Cineol können bei einigen Patienten mit viskösem Sekret hilfreich sein (Evidenzgrad D).

Nach mehreren kontrollierten Studien [187-195] sowie MetaAnalysen [196 - 198] findet sich bei prophylaktischer Gabe in Tagesdosen von 400-1200 mg Acetylcystein eine Reduktion (20-25\%) akuter Exazerbationen während der Wintermonate. Eine Reduktion von Exazerbationen und Beschwerden wurde auch für die 6-monatige orale Gabe von $75 \mathrm{mg}$ Ambroxol bei Patienten mit COPD beschrieben [199]. Im Gegensatz hierzu hat eine aktuelle, groß angelegte, prospektive, doppelblinde, plazebokontrollierte Studie mit einer Tagesdosis von $600 \mathrm{mg}$ N-Acetylcystein [200] keinen wesentlichen Vorteil für die Verumgruppe erbracht: Der jährliche Verlust an $\mathrm{FEV}_{1}$ und die Exazerbationsrate waren in beiden Gruppen gleich. Eine Subgruppenanalyse zeigte einen Effekt auf die Exazerbationsrate bei den Patienten, die nicht mit inhalierbaren Steroiden behandelt wurden. In der Verumgruppe wurde eine Reduktion der Überblähung beobachtet.

Eine Steigerung der Expektoration lässt sich durch die Inhalation von $\beta_{2}$-Sympathomimetika (ggf. in Kombination mit physiologischer oder hypertoner Kochsalzlösung) und durch Theophyllin erzielen. Eine erhöhte Flüssigkeitszufuhr fördert die Expektoration nur bei dehydrierten Patienten. Die Empfehlung großer Trinkmengen ist nicht gerechtfertigt. Sie kann der Dekompensation eines chronischen Cor pulmonale Vorschub leisten (Evidenzgrad D).

\section{Immunmodulatoren}

In einer randomisierten, doppelblind angelegten und plazebokontrollierten Studie führte die Gabe eines Immunmodulators (lyophilisierter normierter Bakterienextrakt) bei COPD-Patienten nicht zu einer Abnahme der Exazerbationsfrequenz. Günstig beinflusst wurde aber der Exazerbationsschweregrad und damit die dadurch bedingten Krankenhausaufenthalte [201]. Eine wei- 
tere plazebokontrollierte Studie [202] zeigte eine signifikante Abnahme der Exazerbationsfrequenz in der Verumgruppe. Da mehr Studien mit ähnlich gutem Design, vergleichbar großen Patientenzahlen und der Dokumentation von Langzeitverläufen zum Einsatz von Modulatoren des Immunsystems bei COPD-Patienten fehlen, kann gegenwärtig die regelmäßige Anwendung von Immunmodulatoren nicht generell empfohlen werden [203].

\section{Antitussiva}

Husten kann Patienten mit COPD stark beeinträchtigen. Bei zunehmendem nicht produktiven Husten sind Antibiotika nicht indiziert. Patienten mit hustenbedingter Störung der Nachtruhe profitieren von der abendlichen Gabe ausreichend hoch dosierter Antitussiva (z.B. $60 \mathrm{mg}$ Codein, $20 \mathrm{mg}$ Dihydrocodein). Die potenziell atemdepressive Wirkung mancher Antitussiva ist bei Patienten mit respiratorischer Globalinsuffizienz $\mathrm{zu}$ beachten. Gegebenenfalls kann die Hustendämpfung mit codeinfreien Antitussiva (z.B. Clobutinol, Noscapin) erfolgen.

Wegen des protektiven Effektes eines erhaltenen Hustenreflexes [204] - besonders wichtig bei Hyperkapnie - kann die regelmäßige Einnahme von Antitussiva bei Patienten mit stabiler COPD nicht empfohlen werden. Ihr Einsatz sollte auf maximal 3 Wochen beschränkt bleiben (Evidenzgrad D). Insbesondere sollten bei Zunahme des Hustens im Rahmen von schweren Exazerbationen der COPD deren Ursachen behandelt und Codein bzw. Narkotika vermieden werden, um eine Atemdepression und die hiermit verbundene Verschlechterung der Hyperkapnie zu vermeiden.

\section{Atemstimulanzien}

Die in früheren Zeiten häufiger verordneten Atemstimulanzien Doxapram und Almitrin sollten angesichts nachgewiesener unerwünschter Effekte bei stabiler COPD nicht eingesetzt werden [205 - 207] (Evidenzgrad B).

\section{Analgetika (Morphin)}

Der Einsatz von Morphin kann bei schwerer Dyspnoe zur Linderung beitragen. Wegen bedeutsamer unerwünschter Effekte (u.a. Atemdepression) sollte der Einsatz auf wenige besonders beeinträchtigte Patienten mit schwerer Atemnot und Hyperventilation beschränkt und unter stationären Bedingungen eingeleitet werden [208-213] (Evidenzgrad C).

\section{Weitere medikamentöse Therapieoptionen}

In Anbetracht unzureichender Daten kann der Einsatz von Antileukotrienen, Nedocromil, DNCG sowie von homöopathischen Therapieverfahren nicht empfohlen werden.

\section{Substitutionstherapie bei}

\section{Alpha-1-Protease-Inhibitor-Mangel}

Patienten mit angeborenem Alpha-1-Protease-Inhibitor-Mangel entwickeln häufig, insbesondere bei chronischem Tabakkonsum, frühzeitig ein Lungenemphysem.

Haupteffekt der Substitutionsbehandlung mit aus menschlichem Plasma gewonnenem Alpha-1-Protease-Inhibitor (Prolastin $\mathrm{HS}^{\circledR}$ ) ist auf der Basis der Auswertungen eines amerikanischen und eines deutschen Fallregisters eine Verlangsamung der Emphysemprogredienz, erkennbar an einer geringeren jährlichen Abnahme der $\mathrm{FEV}_{1}[214-216]$.

Eine Substitutionsbehandlung, z.B. mit wöchentlicher i.v.-Applikation von Alpha-1-Protease-Inhibitor in einer Dosis von
$60 \mathrm{mg} / \mathrm{kg}$ Körpergewicht, kommt unter Berücksichtigung der begrenzten Wirkung und der hohen Therapiekosten nur bei Patienten mit homozygotem Alpha-1-Protease-Inhibitor-Mangel (<35\% des Normwertes), mittelgradiger Funktionseinschränkung ( $30 \%$ Soll $<\mathrm{FEV}_{1}<65 \%$ Soll) und/oder ausgeprägter jährlicher Reduktion der $\mathrm{FEV}_{1}$ (Verlust $\mathrm{FEV}_{1} / \mathrm{Jahr}>50 \mathrm{ml}$ ) in Betracht (Evidenzgrad B).

Bei Patienten mit schwerem Alpha-1-Protease-Inhibitor-Mangel und schwerer Funktionseinschränkung $\left(\mathrm{FEV}_{1} \leq 30 \%\right.$ des Sollwertes) kann hingegen die Substitutionstherapie nicht generell empfohlen werden. Ein dekompensiertes Cor pulmonale ist eine Kontraindikation für diese Substitutionstherapie. Bei Patienten mit schwerem Alpha-1-Protease-Inhibitor-Mangel und normaler Lungenfunktion sowie jährlichem Abfall der $\mathrm{FEV}_{1}$ von weniger als $50 \mathrm{ml}$ ist die Substitutionstherapie ebenfalls nicht erforderlich. Bei den Patienten unter Substitution sollten der Alpha-1-Protease-Inhibitor-Spiegel vor der nächsten Infusion über $35 \%$ des Normwertes liegen.

Unabhängig von einer Substitutionstherapie ist für alle Patienten mit Alpha-1-Protease-Inhibitor-Mangel eine strikte Nikotinkarenz zu fordern. Eine Substitutionstherapie bei Rauchern ist angesichts der Inaktivierung des Alpha-1-Protease-Inhibitors durch Zigarettenrauchen nicht zu rechtfertigen. Eiweißunverträglichkeiten sowie ein kompletter IgA-Mangel sind Kontraindikationen der Substitutionstherapie.

\section{Behandlung der Osteoporose}

Bei Patienten mit COPD kann sich im Krankheitsverlauf eine ausgeprägte Osteoporose entwickeln, die durch Immobilität, mangelhafte Ernährung und die langfristige Einnahme systemischer Glukokortikoide begünstigt wird [217].

Zur Prophylaxe dienen eine calciumreiche Ernährung bzw. die Gabe von 500 - 1000 mg Calcium und 500 - 1000 Einheiten Vitamin D3 täglich. Günstig ist regelmäßige körperliche Bewegung. Immobilen Patienten ist ein isometrisches Übungsprogramm (Osteoporose-Training) zu empfehlen (Evidenzgrad D).

Bei gefährdeten Personen kann eine Risikoabschätzung und Therapiekontrolle mittels Osteodensitometrie der Wirbelsäule erfolgen. In der Therapie der glukokortikoidinduzierten Osteoporose ist der Einsatz auch von Bisphosphonaten erwägenswert [218]. Kontrollierte und randomisierte Studien für den Einsatz von Calcium, Vitamin D, Oestrogenen und Bisphosphonaten für COPD-Patienten liegen nicht vor.

\section{Nicht medikamentöse Therapie}

Nichtmedikamentöse Therapiemaßnahmen haben bei der COPD einen hohen Stellenwert.

\section{Körperliches Training}

Mit zunehmendem Schweregrad der COPD resultiert aus der Belastungsdyspnoe mit weiter abnehmender körperlicher Belastbarkeit infolge körperlicher Schonung und Dekonditionierung von Herz, Kreislauf und Muskulatur eine Abnahme der Lebensqualität mit den Folgen einer zunehmenden sozialen Isolation und häufigem Auftreten einer Depression, die die Belastungsdyspnoe verstärkt ( Abb. 4).

Körperliches Training führt bei COPD-Patienten ab Schweregrad II zur Steigerung der Lebensqualität und Belastbarkeit und zur Verringerung der Exazerbationsrate. Körperliches Training sollte daher Teil der Langzeittherapie sein.

Patienten mit COPD profitieren von körperlichem Training mit einer Steigerung der Belastbarkeit und einer Linderung von Dys- 


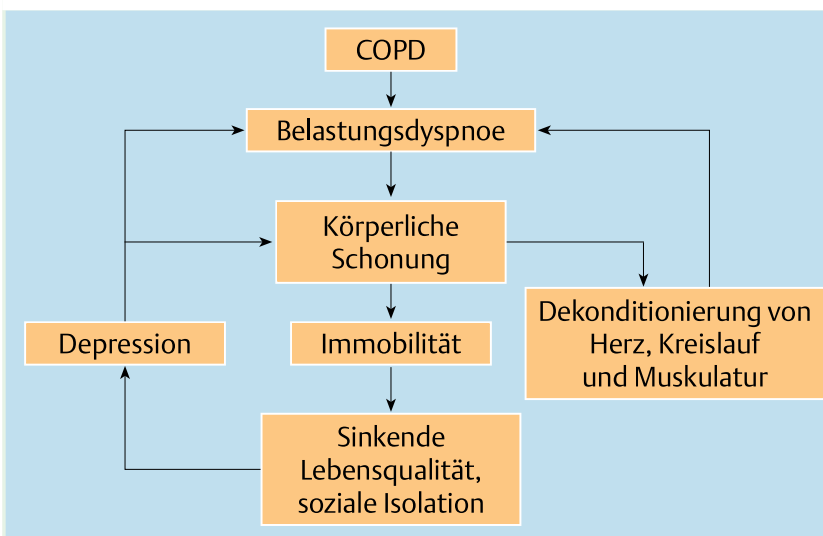

Abb. 4 Auswirkungen der COPD auf körperliche Belastbarkeit, Lebensqualität und Psyche.

pnoe und Ermüdbarkeit (Evidenzgrad A). Trainingseffekte sind für COPD-Patienten aller Schweregrade $[219,220]$ durch randomisierte und kontrollierte Studien belegt [221 - 224]. Positive Effekte werden insbesondere bei Trainingsprogrammen mit einer Dauer von 4-10 Wochen, 3-5 Übungseinheiten pro Woche [225] unter Supervision [226] und hoher Trainingsintensivität nahe der anaeroben Schwelle [224] erzielt. Auch bei fortgeschrittenen Krankheitsstadien werden eine Verringerung der COPD-Symptomatik, eine Steigerung der Belastbarkeit, eine Besserung der Lebensqualität, eine Abnahme der krankheitsbezogenen Depression sowie eine Verringerung der Morbidität und des akutmedizinischen Ressourcenverbrauches gesichert [227,228]. Während die generelle Wirksamkeit der Trainingstherapie der COPD als gesichert angesehen werden kann, besteht bezüglich der Trainingsmodalitäten (Rolle des Krafttrainings, kombinierte Trainingsformen, Steuerung der Trainingsintensität, Atemmuskeltraining u.a.) noch Forschungsbedarf $[229,230]$.

Wesentlich ist die Fortsetzung der Trainingstherapie nach Beendigung intensiver stationärer Rehabilitationsprogramme im ambulanten Bereich, etwa durch Heimtraining (Treppensteigen, Gehtraining) in Verbindung mit der Teilnahme an ambulanten Lungensportgruppen. Das Trainingsprogramm sowie die Überwachung des körperlichen Trainings bei COPD-Patienten sollten in Abhängigkeit vom Schweregrad der Erkrankung gestaltet werden [224].

Isolierte Programme zum Training der Arm- oder Beinmuskulatur können bei Patienten, die ein allgemeines körperliches Training wegen erheblicher Begleitkrankheiten nicht durchführen können, hilfreich sein [224,231]. Mit derartigen Trainingsprogrammen isolierter Muskelgruppen kann deren Kraft verbessert werden. Es liegen jedoch keine Belege dafür vor, dass ein Krafttraining der Arme zu einer verbesserten allgemeinen Belastbarkeit oder einer höheren Lebensqualität führt [231]. Ein isoliertes Training der Inspirationsmuskeln durch Atmung über Widerstände unter Kontrolle der Atemstromstärke kann insbesondere bei Patienten mit ventilatorischer Insuffizienz zu einer Steigerung von Atemmuskelkraft, Ausdauer und allgemeiner Leistungsfähigkeit führen [232] (Evidenzgrad B).

Neuere Untersuchungen zeigen, dass die Besserung der körperlichen Belastbarkeit und des Gesundheitszustandes nach einer einzigen Rehabilitationsmaßnahme über mehr als ein Jahr aufrecht erhalten werden kann [233-235].

Voraussetzung für Langzeiteffekte körperlichen Trainings ist die häusliche oder wohnortnahe Fortsetzung der Bewegungsthera- pie (Evidenzgrad B). Um diese zu gewährleisten, ist ein wohnortnahes ambulantes Rehabilitationsangebot, verbunden mit häuslichem Training, z.B. im Rahmen ambulanter Lungensportgruppen, zu fordern [224].

\section{Patientenschulung}

Die Patientenschulung ist ein wichtiges Therapieelement für alle Schweregrade der Erkrankung, da sie zu einer Steigerung der Effizienz des Managements wesentlich beiträgt (Evidenzgrad D). Jeder Patient mit COPD sollte Zugang zu einem strukturierten, evaluierten und zielgruppenspezifischen Schulungsprogramm erhalten und ärztlicherseits regelhaft zur Teilnahme an der Schulung motiviert werden. Nachschulungen nach spätestens 2 Jahren sind sinnvoll.

Der Stellenwert der alleinigen Patientenschulung im Management der COPD ist bisher nicht abschließend durch randomisierte, kontrollierte Studien belegt. In einer prospektiven, randomisierten, kontrollierten Studie mit einem ambulanten strukturierten Schulungsprogramm für Patienten mit chronisch obstruktiver Bronchitis konnte gezeigt werden, dass zumindest bei Patienten mit leicht- und mittelgradiger COPD im Vergleich zur Kontrollgruppe die Inhalationstechnik gebessert, die Selbstkontrolle der Erkrankung gesteigert, die Zahl akuter Exazerbationen reduziert und bei Steigerung der Lebensqualität die Kosten vermindert werden können [236,237] (Evidenzgrad B).

Patientenschulungen im Rahmen eines umfassenden Selbstmanagementtrainings mit individuellen Anweisungen für Exazerbationen und zum körperlichen Training unter Einsatz telefonischer Nachsorge führten in einer randomisierten, kontrollierten Multicenterstudie innerhalb eines Jahres zu einer signifikanten Reduktion von Krankenhausaufnahmen und Notfallbehandlungen [238]. Dieser Effekt war auch nach 2 Jahren noch nachweisbar [239].

$\mathrm{Zu}$ den wesentlichen Inhalten der Patientenschulung gehören Informationen über Risikofaktoren und deren Reduktion bzw. Elimination, insbesondere der Hinweis auf die Wichtigkeit der Raucherentwöhnung.

Für alle Schweregrade sind das Monitoring von Symptomen, die schweregradadaptierte Selbstmedikation, die Vorbeugung und Behandlung von Exazerbationen und Bronchialinfekten neben korrekter Inhalationstechnik und Wissensvermittlung über die COPD sowie atemerleichternde Stellungen wichtige Lehrinhalte. Für den Schweregrad IV kommen Informationen über Komplikationen, die apparative Therapie mittels Langzeitsauerstoffbehandlung bzw. intermittierende Selbstbeatmung als zusätzliche Lehrinhalte in Betracht.

Die Patientenschulung für COPD-Patienten sollte die individuellen Belange des Patienten und seine Umgebung berücksichtigen. Die Raucherentwöhnung kann während einer pneumologischen Rehabilitation in ein multimodales Schulungsprogramm integriert werden; in ambulanten Programmen sollte sie wegen ihres erheblichen Zeitaufwandes separat von anderen Schulungsprogrammelementen durchgeführt werden, um die positiven Effekte der Gruppeninteraktion nicht durch eine zu lang terminierte Schulung mit der Schwierigkeit des Einhaltens gemeinsamer Termine aller Schulungsteilnehmer zu gefährden.

\section{Physiotherapie}

Hauptziele der physiotherapeutischen Atemtherapie sind eine Erleichterung der erschwerten Atmung in Ruhe und unter Belastung sowie eine Verbesserung der Mukusclearance und des Hustens. 
Diese Atemtherapie wird bei COPD-Patienten zur Senkung der Atemarbeit, zum gezielten Einsatz der Atemmuskulatur, zur Verbesserung der Sekretelimination und der Thoraxbeweglichkeit und damit zur Verbesserung des Gasaustausches eingesetzt [240,241] (Evidenzgrad C). Randomisierte, kontrollierte Studien zum Stellenwert der Atemphysiotherapie in der Behandlung der COPD fehlen.

Mittels Relaxations- und Atemtechniken kann die Atemnot gelindert werden [242]. Atemerleichternde Körperstellungen, z.B. der Kutschersitz, reduzieren erhöhte Atemwegswiderstände durch das Anheben der Atemmittellage, unterstützen die Funktion der Atemhilfsmuskulatur, entlasten den Thorax vom Gewicht des Schultergürtels und vermindern durch die Kombination mit der Lippenbremse den Atemwegskollaps. Therapeutische Körperstellungen, z.B. Dehnlagen, Wärmeapplikation und manuelle Techniken, können zur Ökonomisierung der Atemarbeit beitragen.

Mittels exspiratorisch wirksamer Stenosen, z.B. der dosierten Lippenbremse oder eines Strohhalmstücks, kann der exspiratorische Kollaps bei tracheobronchialer Instabilität durch eine intrabronchiale Druckerhöhung vermindert oder vermieden werden, z.B. der hierdurch bedingte unproduktive Husten beim Treppensteigen.

Atemtechniken mit deutlichen atemsynchronen Bronchialkaliberschwankungen und variierenden exspiratorischen Flüssen mit und ohne exspiratorische Stenosen, z. B. die modifizierte autogene Drainage, stellen eine Möglichkeit der Sekretelimination dar. Auch bieten sich Lagerungen mit Drehungen des Thorax zur Mobilisierung von Sekret unter Nutzung der Schwerkraft und veränderter Perfusions- und Ventilationsverhältnisse an. Die Lagerungsdrainage kann in Kombination mit Atemtechniken sowie mit Vibrationen und Kompressionen des Thorax während der Exspiration zu einer besseren Sekretelimination führen. Die Lagerungsdrainage sollte bei Patienten mit Sekretretention und Sputummengen von mehr als $30 \mathrm{ml}$ pro Tag eingesetzt werden [242]. Hierbei ist zu berücksichtigen, dass die Messung der Sputumproduktion schwierig ist. Bei geringeren Sputummengen liegen keine Belege dafür vor, dass die Lagerungsdrainage bei akuten Exazerbationen [243] oder bei unkomplizierten Pneumonien [244] hilfreich ist.

Die Perkussion des Thorax wird wegen der hohen notwendigen Perkussionsfrequenz seltener als in früheren Jahren eingesetzt [245].

Das Huffing, eine Atemtechnik mit vertiefter Inspiration und anschließend forcierter Exspiration bei offener Glottis, ist in der Lage, Sekret aus den zentralen und kleineren Atemwegen zu entfernen. Ein Halt am Ende der Inspiration für etwa 2 - 3 Sekunden erhöht die kollaterale Ventilation [246]. Im Gegensatz zur Situation beim Husten erleiden COPD-Patienten beim Huffing keinen Tracheobronchialkollaps [247].

\section{Hilfsmittel zur Sekretelimination}

Randomisierte, kontrollierte Studien zum Stellenwert von Hilfsmitteln mit und ohne Oszillationen zur Sekretelimination liegen nicht vor. Handliche Geräte (Strohhalmstück, VRP1 ${ }^{\circledR}$-Flutter, RCCornet $^{\circledR}$, acapella choice ${ }^{\circledR}$, Pari-PEP-System ${ }^{\circledR}$, PEP-Maske $\left.{ }^{\circledR}\right)$, mit deren Hilfe ein positiver exspiratorischer Druck (PEP) aufgebaut wird, sind auf der einen Seite in der Lage, Bronchialverschlüsse durch Instabilität der Bronchialwand zu verhindern oder zumindest zu verringern, auf der anderen Seite durch den Überdruck und die nachfolgende Erweiterung der Bronchien Sekret von den Bronchialwänden zu lösen, das mittels Huffing aus dem
Bronchialbaum entfernt werden kann. Auf den produktiv-ineffektiven Husten infolge der tracheobronchialen Instabilität kann somit erheblich Einfluss genommen werden. Die Effektivität der VRP1 ${ }^{\circledR}$-Flutter ist nicht nur bei Bronchiektasie und Mukoviszidose, sondern auch bei der COPD in Studien mit geringer Teilnehmerzahl belegt [248,249] (Evidenzgrad C).

Das RC-Cornet ${ }^{\circledR}$ hat gegenüber dem VRP1 ${ }^{\circledR}$-Flutter den Vorteil, dass es von der Schwerkraft unabhängig ist und somit in jeder Körperlage benutzt werden kann. Der Patient kann die für ihn günstigste Position und Einstellung des Widerstandes selbst ermitteln [250]. Zeitsparend lässt sich die Anwendung dieser Hilfsmittel mit Inhalationen, Drainagelagerungen und weiteren physiotherapeutischen Techniken kombinieren [251].

\section{Ernährung}

Übergewicht und Untergewicht beeinflussen Symptomatik und Prognose von Patienten mit COPD. Als Gewichtsverlust ist eine Abnahme des Körpergewichts um mehr als 10\% in den letzten 6 Monaten oder um mehr als 5\% im letzten Monat zu werten. In diesen Fällen ist es das Ziel der Ernährungstherapie, mittels oraler Nährstoffzufuhr, ggf. Ernährungssupplementierung, eine Gewichtszunahme zu erreichen. Sinnvoll erscheint die Kombination der Ernährungstherapie mit körperlichem Training, z.B. im Rahmen eines Rehabilitationsprogramms.

Die meisten Ernährungsempfehlungen basieren auf kleinen randomisierten Studien. Etwa 25\% der Patienten mit mittelgradiger und schwerer COPD zeigen eine Reduktion des body-mass-index und der fettfreien Masse [252 - 255]. Auch bei normalgewichtigen Patienten kann die fettfreie Masse erniedrigt sein [253]. Unterernährung kann die Prognose, unabhängig vom Ausmaß der Obstruktion, beeinträchtigen [254,256-258]. Das Untergewicht korreliert bei COPD-Patienten mit Muskelschwäche [252], eingeschränkter Belastbarkeit [259] und verminderter Lebensqualität [260]. In einer prospektiven Untersuchung konnten bei der Mehrzahl der untergewichtigen Patienten mittels einer hochkalorischen Nahrungszufuhr innerhalb von 8 Wochen eine Gewichtszunahme und auch eine Besserung der Prognose erreicht werden [80]. Die mittels Kostaufbau mögliche Gewichtskorrektur untergewichtiger Patienten kann zu einer Besserung der Symptome führen.

Dennoch muss festgehalten werden, dass es gegenwärtig nur wenige qualitativ hochwertige randomisierte Doppelblindstudien zur Ernährungstherapie bei COPD gibt. Eine Meta-Analyse zur Effektivität alleiniger Ernährungsinterventionen (Zusatznahrung über mindestens 2 Wochen) konnte keine eindeutig signifikant positiven Effekte auf Anthropometrie, Lungenfunktion oder Belastbarkeit aufzeigen [261,262].

Bezüglich der Selektionskriterien der von einer Ernährungstherapie profitierenden Patienten, der Auswirkungen einer entsprechenden, das Gewicht steigernden Kost auf Morbidität und Lebensqualität, der optimalen Zusammensetzung der Nahrung sowie bezüglich Kosten und Nutzen einer oralen Zusatzernährung ist die Datenlage noch unzureichend.

Sollten die Patienten infolge Atemnot zu geringe Nahrungsmengen aufnehmen, sind kleine, häufige Mahlzeiten zu empfehlen. Falls notwendig, sollte das Gebiss saniert werden.

Bei Verlust an Muskelkraft infolge Untergewicht kann die Atemmuskelkraft durch gesteigerte Kalorienzufuhr bei einem Teil der Patienten gebessert werden [263-265]. Meist reicht die alleinige Zufuhr von Kalorien nicht aus, sie sollte durch körperliches Training bzw. Training der Atemmuskeln ergänzt werden. Dies- 
bezüglich liegen jedoch keine Studien an großen Patientenzahlen vor.

Bei übergewichtigen Patienten führt eine Gewichtsreduktion zu einer Abnahme des Energiebedarfs bei körperlicher Belastung sowie zu einer Besserung der Atemmechanik und damit zu einer leichteren Bewältigung der im Alltag anfallenden körperlichen Aktivitäten. Diäten zur Gewichtsreduktion können mit einer Beschränkung der täglichen Kalorienaufnahme auf 1200 - 1500 Kalorien erfolgreich durchgeführt werden.

Der Zusatz von Vitaminen oder Mineralstoffen ist bei ausgewogener Ernährung nicht erforderlich. Der Stellenwert der Gabe von Anabolika bei stark untergewichtigen COPD-Patienten kann noch nicht mit hinreichender Sicherheit beurteilt werden [266-268].

\section{Pneumologische Rehabilitation}

Die ambulante oder stationäre Rehabilitation [230] soll insbesondere bei COPD-Patienten ab einem Schweregrad II und auch in höherem Lebensalter als wirksame Komponente des langfristig ausgerichteten Managements der COPD durchgeführt werden. Die stationären Rehabilitationsmaßnahmen müssen dauerhaft durch weitere ambulante Maßnahmen ergänzt werden, z. B. den ambulanten Lungensport. Hauptziele der Rehabilitation sind die Linderung der physischen und psychischen Beeinträchtigung des Patienten, die Steigerung der Lebensqualität mit Wiederherstellung der bestmöglichen Leistungsfähigkeit sowie die Förderung der sozialen Reintegration.

Um diese Ziele zu erreichen, bedarf es eines interdisziplinären und multimodalen Ansatzes unter Einbeziehung von Ärzten, Psychologen, Atemphysiotherapeuten, Sporttherapeuten und Ernährungsberatern $[269,270]$.

Komponenten der pneumologischen Rehabilitation

Neben einer Optimierung der Pharmakotherapie sind weitere Inhalte der Rehabilitation:

Tabakentwöhnung,

körperliches Training,

Patientenschulung,

Atemphysiotherapie,

Ergotherapie,

Ernährungsberatung,

Hilfsmittelversorgung,

soziale Betreuung,

psychosoziale Diagnostik, Beratung und Therapie sowie

die sozialmedizinische Begutachtung.

\section{Effekte der pneumologischen Rehabilitation bei Patienten mit COPD}

Die gesicherten Erfolge der Rehabilitation sind in $\bullet$ Tab. 9 zusammengefasst. Hierbei wurden umfassende interdisziplinäre Rehabilitationsprogramme und deren Analysen berücksichtigt [221-223].

Das regelhafte Einbeziehen der medikamentösen Therapie in die pneumologische Rehabilitation - wie in Deutschland üblich kann den Rehabilitationserfolg insbesondere bezüglich der Besserung der Dyspnoe, der Belastbarkeit und der Lebensqualität zusätzlich steigern [138].

\section{Auswahl der Patienten}

Wesentlich für den Erfolg der pneumologischen Rehabilitation ist der motivierte Patient. Indiziert sind Rehabilitationsprogramme insbesondere für COPD-Patienten der Schweregrade II-IV
Tab. 9 Gesicherte positive Effekte der pneumologischen Rehabilitation $[2,221-223]$

\begin{tabular}{|c|c|}
\hline Nutzen & Evidenzgrad \\
\hline gesteigerte körperliche Leistungsfähigkeit & A \\
\hline Abnahme der Atemnot & A \\
\hline $\begin{array}{l}\text { Steigerung der krankheitsspezifischen Lebens- } \\
\text { qualität }\end{array}$ & A \\
\hline $\begin{array}{l}\text { Reduktion der Anzahl und Dauer von Kranken- } \\
\text { hausaufenthalten }\end{array}$ & A \\
\hline $\begin{array}{l}\text { Abnahme von COPD assoziierter Angst und De- } \\
\text { pression }\end{array}$ & A \\
\hline $\begin{array}{l}\text { Kraft- und Ausdauertraining der oberen Extremi- } \\
\text { tät verbessert die Funktion der Arme }\end{array}$ & B \\
\hline $\begin{array}{l}\text { positive Effekte eines Trainingsprogramms über- } \\
\text { dauern die Trainingsperiode }\end{array}$ & B \\
\hline Lebensverlängerung & B \\
\hline $\begin{array}{l}\text { Atemmuskeltraining ist effektiv, insbesondere in } \\
\text { Kombination mit einem allgemeinen körperli- } \\
\text { chen Training }\end{array}$ & C \\
\hline psychosoziale Intervention ist hilfreich & C \\
\hline
\end{tabular}

$[2,4]$ auch für Raucher [271] insbesondere dann, wenn sie an Entwöhnungsprogrammen teilnehmen.

Erfahrungsgemäß nehmen Raucher seltener als Nichtraucher das komplette Angebot eines pulmonalen Rehabilitationsprogramms in Anspruch [272].

COPD-Patienten mit Z.n. Aufenthalt im Akutkrankenhaus infolge einer schweren Exazerbation sind mit einer hohen Morbidität und Mortalität belastet. So mussten in einer Studie [273] von 1016 Patienten, die wegen einer COPD stationär aufgenommen wurden, 446 Patienten insgesamt 754-mal in den 6 Monaten nach Entlassung erneut stationär behandelt werden. Auch in einer prospektiven Studie aus Spanien [274] mussten von 430 konsekutiven COPD-Patienten, die wegen ihrer akuten Verschlechterung stationär behandelt wurden, $63 \%$ innerhalb eines mittleren Beobachtungszeitraums von 1,1 Jahren mindestens 1x erneut stationär behandelt werden, $29 \%$ verstarben innerhalb dieses Zeitraumes. Daher sollte speziell bei diesen Patienten die Indikation zur ambulanten und stationären Anschlussrehabilitation regelhaft geprüft werden, zumal es speziell für die Effizienz der pneumologischen Rehabilitation direkt nach Behandlung der akuten Exazerbation zunehmende Evidenz gibt [275 - 277]. Generell besteht eine Indikation zur Rehabilitation, wenn trotz adäquater Krankenbehandlung körperliche oder psychosoziale Krankheitsfolgen [278] persistieren, welche alltagsrelevante Aktivitäten und die Teilhabe am normalen, privaten, öffentlichen oder beruflichen Leben behindern $[105,279]$.

Wichtige spezielle Indikationen sind z. B.:

alltagsrelevante, persistierende COPD-Symptome [280],

Gefährdung der Erwerbsfähigkeit [281,282],

drohende Pflegebedürftigkeit [283],

alltagsrelevante psychosoziale Krankheitsfolgen (Depression, Angst, Rückzugstendenz),

Notwendigkeit von rehaspezifischen nichtmedikamentösen Therapieverfahren, wenn diese ambulant nicht im erforderlichen Umfang erfolgen können, z. B. körperliches Training, Physiotherapie, Patientenschulung oder psychosoziale Hilfen.

\section{Organisation der pneumologischen Rehabilitation}

In der Bundesrepublik Deutschland ist die medizinische Rehabilitation in das gegliederte System der sozialen Sicherung mit seinen unterschiedlichen Zuständigkeiten und Trägerstrukturen 
eingebunden. Träger und Leistungsrahmen der Rehabilitation sind gesetzlich umfassend geregelt. In Übereinstimmung mit $\S \S 3,4$ und 8 SGB IX formuliert das deutsche Renten-, Krankenund Unfallversicherungsrecht für den chronisch Atemwegskranken, der Krankheitsfolgen aufweist, ausdrücklich einen Anspruch auf Rehabilitation („Leistungen zur Teilhabe“).

In enger Abstimmung zwischen Patient, Hausarzt und Pneumologen können auch ambulante rehabilitative Maßnahmen (Training, Schulung, Tabakentwöhnung, Physiotherapie) wohnortnah, z.B. nach Akutbehandlungen, durchgeführt oder einer stationären Rehabilitation sowohl vor- als auch nachgeschaltet werden. Ein flächendeckendes Angebot an solchen ambulanten rehabilitativen Maßnahmen für COPD-Patienten liegt in Deutschland jedoch z.Z. noch nicht vor.

Voraussetzung für die Einleitung einer stationären Rehabilitationsmaßnahme sind ein Antrag des Patienten sowie ein Attest bzw. eine Rehabilitationsverordnung des behandelnden Arztes. Die stationäre Rehabilitation wird nach Abstimmung zwischen Patient, Hausarzt, Pneumologen und Akutklinik oder Lungenfachklinik eingeleitet. Sie ist insbesondere bei unzureichender Besserung nach ambulanter Behandlung, vor und nach Durchführung ambulanter rehabilitativer Maßnahmen oder nach einer Krankenhausbehandlung als Anschlussrehabilitation zu erwägen. Zur Aufrechterhaltung dauerhafter Effekte können stationäre Rehabilitationen auch wiederholt werden.

Auch zur Aufrechterhaltung der Effekte umfassender stationärer Rehabilitationsprogramme ist eine Rehabilitationsnachsorge mit Teilkomponenten wie etwa der Bewegungstherapie im Rahmen ambulanter Lungensportgruppen, verbunden mit Elementen der Patientenschulung, sinnvoll $[269,284]$ (Evidenzgrad D). Positive Effekte bei COPD-Patienten sind sowohl für stationäre (Evidenzgrad A) als auch für ambulante (Evidenzgrad A) Rehabilitationsprogramme dokumentiert [223,224,261,285,286].

\section{Langzeitsauerstofftherapie (LOT)}

Die Langzeitbehandlung mit Sauerstoff (LOT) ist bei Patienten mit chronischer Hypoxämie im Stadium IV der COPD entsprechend den internationalen Empfehlungen [5-10] sowie den Leitlinien zur LOT der DGP [287] indiziert (Evidenzgrad A).

Primäre Ziele sind eine Anhebung des arteriellen Sauerstoffpartialdrucks auf Werte über $60 \mathrm{~mm} \mathrm{Hg}$, was eine adäquate Sauerstoffversorgung des Gewebes ermöglicht, sowie eine Entlastung der Atemmuskulatur durch einen bei Sauerstoffzufuhr verminderten Bedarf an Ventilation. Die LOT führt bei Patienten mit chronischer respiratorischer Insuffizienz infolge COPD bei Anwendung über 16-24 Stunden pro Tag zu einer Verbesserung der Prognose [288, 289] (Evidenzgrad A).

Weitere Effekte sind eine Verringerung der Progression der pulmonalen Hypertonie bei COPD [290] sowie positive Auswirkungen auf Hämatokrit, Belastbarkeit [291], Atemmechanik und neuropsychologische Parameter [293]. Bei Patienten mit Belastungshypoxämie können Kraft und Funktion der Muskulatur durch die Sauerstoffgabe bei körperlicher Belastung gefördert werden. Die positiven Effekte der LOT sind um so ausgeprägter, je länger die tägliche Sauerstoffinsufflation erfolgt (Evidenzgrad A, Empfehlungsgrad A).

Bei Patienten mit schwerer respiratorischer Insuffizienz ist die Indikation für die LOT gegeben, wenn in der stabilen Phase der Erkrankung nach Optimierung der Pharmakotherapie folgende Entscheidungskriterien erfüllt sind:

$\mathrm{PaO}_{2} \leq 55 \mathrm{~mm} \mathrm{Hg}$ mit und ohne Hyperkapnie.
$\mathrm{PaO}_{2}$-Werte zwischen $55 \mathrm{~mm} \mathrm{Hg}$ und $59 \mathrm{~mm} \mathrm{Hg}$ bei Nachweis einer pulmonalen Hypertonie, von peripheren Ödemen als Hinweis auf eine Herzinsuffizienz oder bei einer Polyglobulie (Hämatokrit > 55\%).

Die Indikation zur LOT kann anhand der am Tage gemessenen $\mathrm{PaO}_{2}$-Werte gestellt werden. In die Entscheidung sollten auch Messungen während des Treppensteigens oder während eines Gehtestes einfließen. Bei belastungsinduzierter Hypoxämie kann die Dyspnoe durch Verordnung von tragbaren Systemen und die Einstellung höherer Sauerstoffflussraten vermindert werden.

Ein begrenzter Anstieg des $\mathrm{PaCO}_{2}$ auf $\leq 60-70 \mathrm{~mm}$ Hg unter Inhalation von Sauerstoff ist keine Kontraindikation gegen die Langzeitsauerstofftherapie, sofern die Werte nicht stetig ansteigen. Primär hyperkapnische Patienten zeigen sogar bessere Effekte unter der LOT bezüglich Reduktion von Morbidität und Mortalität als chronisch hypoxämische Patienten ohne Hyperkapnie [287] (Evidenzgrad A).

Vorschläge zur Auswahl der Sauerstoffapplikationssysteme (Konzentrator, Flüssigsauerstoff, Sauerstoffdruckflasche) sowie die Modalitäten der Verordnung sind den Leitlinien zur LOT [287] zu entnehmen, ebenso Angaben zur Auswahl geeigneter Patienten und Hinweise für die Durchführung von Verlaufskontrollen.

Von den verfügbaren Systemen hat sich der Sauerstoffkonzentrator gegenüber Sauerstoffflaschen und Flüssigsauerstoffsystemen als preisgünstigstes System erwiesen [292 - 294]. Unter Berücksichtigung der Stromkosten und der fallenden Preise für Flüssigsauerstoffsysteme sind Änderungen der Preisrelationen in Zukunft zu erwarten.

Bei der Verordnung muss die vom Arzt getroffene Wahl des Applikationssystems - Sauerstoffkonzentrator für wenig bewegliche Patienten, Flüssigsauerstoffsysteme für Patienten mit guter Mobilität - gegenüber dem Kostenträger begründet werden. Die Langzeitnutzung durch die Patienten ist zu überprüfen.

\section{Heimbeatmung}

Bei Patienten mit COPD ist die Atemmuskulatur durch die Erhöhung der Atemarbeit schon bei Ruheatmung vermehrt beansprucht infolge

der Atemwegsobstruktion,

der erhöhten Ventilation zur Kompensation der emphysembedingten Gasaustauschstörung,

ungünstiger geometrischer Verhältnisse für die Kraftentfal-

tung der Atemmuskeln (Zwerchfelltiefstand).

Dem erhöhten Bedarf an Leistung der Atemmuskeln steht eine Verminderung ihrer Leistungsfähigkeit infolge von Lungenüberblähung, (evtl. glukokortikoidbedingter) Muskeldystrophie $[173,174]$ und (bei kachektischen Patienten) verminderter Energiezufuhr [295] gegenüber.

Führt die chronische Überlastung der Atemmuskulatur zur Atemmuskelermüdung, so ist die Heimbeatmung zu erwägen. Bei chronischer ventilatorischer Insuffizienz wird die Indikation zur intermittierenden nichtinvasiven Beatmung als Heimbeatmung dann gestellt, wenn alle konservativen Behandlungsmöglichkeiten ausgeschöpft sind und der Patient weiterhin hyperkapnisch ist [296].

Bei arterieller Hypoxämie mit leichter Hyperkapnie $\left(\mathrm{PaCO}_{2}\right.$ $\leq 50 \mathrm{~mm} \mathrm{Hg}$ ) wird zunächst eine LOT durchgeführt, die über eine Abnahme des Atemminutenvolumens die Atemmuskulatur entlastet. Bei fortbestehender schwerer Symptomatik, insbesondere Ruhedyspnoe, massiv eingeschränkter Belastbarkeit, Kon- 
zentrationsstörungen und Beeinträchtigung der Vigilanz und $\mathrm{PaCO}_{2}$-Werten über $50 \mathrm{~mm} \mathrm{Hg}$ sollte die intermittierende Selbstbeatmung in Betracht gezogen werden (Evidenzgrad D). Die Beatmung sollte nichtinvasiv über Nasen- oder Mund-Nasen-Masken erfolgen.

Die Einleitung der nichtinvasiven Beatmung sowie die Einstellung finden in entsprechend spezialisierten Krankenhausabteilungen statt.

Zur möglichst kompletten Entlastung der überlasteten Atemmuskulatur wird eine kontrollierte Beatmung vorgezogen, wobei unter Beatmung normale bis leicht erniedrigte $\mathrm{PaCO}_{2}$-Werte anzustreben sind. Teilentlastende Beatmungsformen haben den Vorteil der häufig besseren Akzeptanz bzw. leichteren Adaptation. Sie führen aber nur zu einer inkompletten Entlastung der Atemmuskulatur und damit nur zu einem geringen Erholungseffekt. Sie können daher nur in leichteren Fällen angewandt werden.

Die Beatmungsdauer, die in der Regel bei 8-14 Stunden pro Tag liegt, muss individuell nach Symptomatik, arteriellen Blutgasen und Inspirationsdrücken ermittelt werden. Anzustreben ist ein nahezu normaler $\mathrm{PaCO}_{2}$ bei Spontanatmung, der bei guten Erholungseffekten der Atemmuskeln auch erreicht werden kann. Empfehlenswert ist eine nächtliche Beatmung, damit am Tage möglichst viel beatmungsfreie Zeit verfügbar ist.

Die Ergebnisse der Heimbeatmung bei COPD weisen eine gegenüber neuromuskulären Erkrankungen, Skoliose bzw. posttuberkulösen Syndromen relativ schlechte Prognose mit einer 5-Jahresletalität von etwa 50-60\% auf [297]. Zusammen mit der LOT kann die nichtinvasive Beatmung zu einer deutlichen Besserung der arteriellen Blutgase am Tage, der Effizienz des Nachtschlafs und der Lebensqualität führen [298] (Evidenzgrad B).

Aufgrund der vorliegenden Daten kann die nichtinvasive Beatmung für den Einsatz bei Patienten mit chronischer ventilatorischer Insuffizienz infolge COPD noch nicht generell empfohlen werden. Bei Subgruppen, insbesondere bei Patienten mit ausgeprägter Hyperkapnie am Tage, sollte sie jedoch zum Einsatz kommen [299].

\section{Operative Therapieverfahren}

\section{Bullektomie}

Bei großen Bullae, die mehr als 1/3 eines Lungenflügels einnehmen und das benachbarte Gewebe komprimieren, kann die Bullektomie zu einer Besserung der Lungenfunktion und zu einer Abnahme der Dyspnoe führen [300] (Evidenzgrad B). Gelegentlich kann die Indikation auch bei Hämoptysen oder rezidivierenden Infektionen gestellt werden.

Vor der Entscheidung zur Bullektomie sollten eine Bronchoskopie, ein Computertomogramm des Thorax (HR-CT), Lungenfunktionstests unter Einschluss der arteriellen Blutgase, die Messung der CO-Diffusionskapazität, die Bestimmung der funktionellen Residualkapazität mittels Helium-Methode und ein Perfusionsszintigramm der Lunge durchgeführt werden.

Gute Ergebnisse des chirurgischen Eingriffs sind bei normaler oder nur gering reduzierter Diffusionskapazität, dem Nachweis von komprimiertem Lungengewebe im Randbereich der Bullae und dem Fehlen einer signifikanten Hypoxämie zu erwarten [301].

Die Bullektomie kann im Rahmen einer videoassistierten oder offenen Thorakotomie, bei beidseitigen Bullae auch mittels Ster- notomie erfolgen. Bei schwerem, generalisiertem Lungenemphysem ist dieser Eingriff nicht indiziert.

\section{Lungenvolumenreduktion}

Das von Cooper [302] etablierte Konzept der operativen Lungenvolumenreduktion (LVR) beim Lungenemphysem beinhaltet die Resektion von stark emphysematös veränderten Lungenarealen mit dem Ziel, die Lungenüberblähung zu reduzieren, die Dyspnoe zu lindern und die Lungenfunktion zu bessern. Eine randomisierte nordamerikanische Studie (NETT-Studie) [303] prüfte an 1033 Patienten die LVR gegen eine konservative Therapie. Dabei waren die Endpunkte Lebensqualität, Belastbarkeit und Mortalität. Insgesamt lag die 3-Monats-Sterblichkeit in der operierten Gruppe mit 7,9\% deutlich höher als in der konservativ behandelten Kohorte (1,3\%). Ein signifikanter Vorteil bezüglich Belastbarkeit und Prognose lag nur in einer Subgruppe mit Oberlappen-betontem Emphysem und eingeschränkter Belastbarkeit vor. Zur in der Studie verwendeten Definition der eingeschränkten Belastbarkeit ( $<25 \mathrm{~W}$ bei Frauen, $<40 \mathrm{~W}$ bei Männern bei einem definierten ergometrischen Rampen-Protokoll) ist anzumerken, dass schwersteingeschränkte Patienten (6 min-Gehstrecke $<140 \mathrm{~m}$ ) ausgeschlossen wurden. Somit sollte die LVR nur dieser kleinen Subgruppe offeriert werden. Weiter sind definierte Ausschlusskriterien zu beachten: Bronchiektasen, $\mathrm{FEV}_{1}$ $<20 \%$ des Sollwertes, manifeste ventilatorische Insuffizienz (persistierende respiratorische Globalinsuffizienz mit $\mathrm{PaCO}_{2}$ $>55 \mathrm{~mm} \mathrm{Hg}$ oder nasale Beatmung), pulmonale Hypertonie (rechtsventrikulärer Druck > $50 \mathrm{~mm} \mathrm{Hg}$ in Ruhe), homogene Verteilung des Emphysems, homozygoter Alpha-1-Protease-Inhibitor-Mangel, relevante Komorbiditäten (z. B. koronare Herzkrankheit, schwere linksventrikuläre Funktionseinschränkung, Tumorleiden) (Evidenzgrad A).

Wie auch bei der Lungentransplantation können nur Patienten für die Operation berücksichtigt werden, bei denen präoperativ eine Rehabilitation [304] erfolgte und alle medikamentösen und nicht medikamentösen Therapieoptionen ausgeschöpft wurden. Weiter ist eine mehrmonatige Rauchabstinenz zwingend erforderlich.

In Studien werden gegenwärtig endoskopische Verfahren zur Emphysemtherapie (EET) geprüft. Prinzipien sind hierbei die endobronchiale Okklusion, die Implantation von Ventilsystemen [305] oder die Schaffung eines Bypass zur kollateralen Ventilation. Ergebnisse randomisierter Studien gegen Plazebo liegen bislang für keines der Verfahren vor. Dem gegenüber der chirurgischen LVR (3-Monats-Mortalität in erfahrenen Zentren unter $5 \%)$ niedrigeren Risiko des Eingriffs müssen spezifische Komplikationen der EET wie Pneumothorax, Pneumonien oder bronchiale Blutung gegenübergestellt werden. Aktuell hat die EET außerhalb von Studien keinen Stellenwert in der Emphysembehandlung.

\section{Lungentransplantation}

Weltweit stellt die COPD die häufigste Indikation zur Lungentransplantation dar (in Deutschland etwa 25\% der Empfänger, ca. 60 Patienten pro Jahr) [306,307]. Randomisierte Studien zur Klärung der Frage, ob die Lungentransplantation (LTx) die Prognose von COPD-Patienten im Terminalstadium verbessert, liegen nicht vor. Im Gegensatz zu einer früheren nordamerikanischen retrospektiven Analyse (mit Einschluss von weniger schwer erkrankten Patienten) [308] hat eine britische retrospektive Untersuchung Hinweise dafür erbracht, dass die LTx bei präoperativ funktionell deutlich schlechteren Patienten einen Überlebens- 


\begin{tabular}{|lll|}
\hline & LVR & LTx \\
\hline Lungenfunktion & $\mathrm{FEV}_{1}: 25-45 \%$ Soll & $\mathrm{FEV}_{1}:<25 \%$ Soll \\
\hline Bildgebung & oberlappenbetontes Emphysem & $\begin{array}{l}\text { panlobuläres Emphysem, Alpha- } \\
\text {-Protease-Inhibitor-Mangel }\end{array}$ \\
\hline 6-Minuten-Gehstrecke & $>150 \mathrm{~m}$ & $<150 \mathrm{~m}$ \\
\hline Blutgasanalyse & $\mathrm{PaCO}_{2}<55 \mathrm{~mm} \mathrm{Hg}$ & $\mathrm{PaCO}_{2}>55 \mathrm{~mm} \mathrm{Hg}$ \\
\hline Echokardiographie & RVP systolisch $<50 \mathrm{~mm} \mathrm{Hg}$ & $\mathrm{RVP}$ systolisch $>50 \mathrm{~mm} \mathrm{Hg}$ \\
\hline
\end{tabular}

Tab. 10 Kriterien zur Differenzialindikation für Lungenvolumenreduktion (LVR) und Lungentransplantation (LTx) bei Patienten mit ausgeprägtem Lungenemphysem vorteil mit sich bringt [309]. Es gilt, im Kollektiv der COPD Patienten Kandidaten mit ungünstigen Prognosekriterien zu selektionieren und ggf. der LTx zuzuführen. Neben der Prognoseverbesserung hat die LTx auch die Verbesserung der Lebensqualität [310] zum Ziel. Eine LTx kommt nur dann in Betracht, wenn alle anderen Therapieverfahren ausgeschöpft worden sind. Dies beinhaltet auch LOT, intermittierende Heimbeatmung, Ernährungs- und Trainingstherapie. Voraussetzung für die Aufnahme auf die Warteliste ist eine dokumentierte mindestens 6-monatige Abstinenz vom Tabakrauchen. Es gilt in den meisten Zentren eine Altersobergrenze von 60 (in Ausnahmen 65) Jahren. Eine durchschnittlich 2-jährige Wartezeit vor Verfügbarkeit eines Spenderorgans ist zu berücksichtigen. Kontraindikationen sind relevante extrapulmonale Komorbiditäten (z.B. KHK, BMI $>30 \mathrm{~kg} / \mathrm{m}^{2}$, Niereninsuffizienz, Leberzirrhose) [310].

Allgemein akzeptierte Kriterien für die Aufnahme auf die Warteliste zur Transplantation sind eine $\mathrm{FEV}_{1}<25 \%$ Soll, ein pulmonaler Hochdruck (mittlerer pulmonalarterieller Druck $\geq 25 \mathrm{~mm} \mathrm{Hg}$ ) und eine respiratorische Globalinsuffizienz $\left(\mathrm{PaCO}_{2} \geq 50 \mathrm{~mm} \mathrm{Hg}\right.$, $\mathrm{PaO}_{2}<55 \mathrm{~mm} \mathrm{Hg}$ ).

Gehäufte Exazerbationen ( $>3 / \mathrm{Jahr}$, insbesondere mit Hospitalisation), Untergewicht, weibliches Geschlecht, hoher Sauerstoffbedarf und stark eingeschränkte Belastbarkeit scheinen negative Prognosekriterien für fortgeschrittene COPD-Patienten zu sein [311].

In den aktuell publizierten Empfehlungen einer Arbeitsgruppe zur Selektion von LTX-Kandidaten mit COPD wird dem BODE-Index der gesundheitsspezifischen Lebensqualität eine wichtige Rolle als unabhängigem Prädiktor für die Mortalität zugeschrieben [312]. Hierbei ist kritisch anzumerken, dass der BODE-Index mit der Fragestellung der Selektion von Transplantationskandidaten nicht validiert wurde. Das Kollektiv, an dem er entwickelt wurde, war durchschnittlich 66 Jahre alt und multimorbide, entsprach also gerade keinem Transplantationskandidatenkollektiv. Von deutschen Experten wird deshalb der BODE-Index als Selektionskriterium zur Aufnahme auf die Warteliste abgelehnt. In den BODE-Index gehen 3 von 4 Parametern ein, die beeinflussbar sind (BMI, 6-Minuten-Gehstrecke und Dyspnoe-Empfinden). Der Index erleichtert in der klinischen Praxis Patienten zu identifizieren, die Interventionsbedarf (z.B. Rehabilitation, Ernährungstherapie) haben. Wenn diese Maßnahmen ausgeschöpft sind, sollte auch die Option der Transplantation geprüft werden. Die Messung der Diffusionskapazität allein ist bei schweren obstruktiven Ventilationsstörungen viel zu unzuverlässig, als dass aus Resultaten dieser Messung Transplantationsentscheidungen getroffen werden sollten.

Für Transplantationskandidaten werden eine hohe Motivation, eine effiziente, vorbereitende Rehabilitations- und Trainingstherapie sowie psychosoziale Stabilität vorausgesetzt.

Besonders ungünstig ist der Lungenfunktionsverlauf bei homozygotem Alpha-1-Protease-Inhibitor-Mangel. Bei diesen meist jüngeren Patienten sollte frühzeitig Kontakt mit einem Transplantationszentrum aufgenommen werden.
Die Ergebnisse für Einzel- und Doppellungentransplantation unterscheiden sich nicht wesentlich für die Altersgruppe der über 60-Jährigen, während in einer Analyse von 2260 Transplantationen wegen COPD aus dem ISHLT-Register 1991 - 1997 [313] in der Altersgruppe der unter 50-Jährigen die 5-Jahres-Überlebensrate nach Doppellungentransplantation mit 68\% gegenüber der nach Einzellungentransplantation mit $43 \%$ deutlich besser war. Ein ähnliches Bild ergab sich auch in der Altersgruppe der 50 - 60-Jährigen mit 60\% gegenüber $40 \%$ zugunsten der Doppellungentransplantation [314].

Für die Altersgruppe der über 60-Jährigen ist die Einzellungentransplantation eine mit reduziertem operativen Risiko durchführbare Maßnahme, außerdem erhöht sie die Verfügbarkeit von Spenderorganen. Zu beachten ist beim Operationsverfahren der Einzellungentransplantation bei Überblähung der nativen Lunge eine postoperative Kompression der transplantierten Lunge. Eine quantitative Ventilations-Perfusions-Szintigraphie (seitengetrennt) ist in diesen Fällen präoperativ obligat. Bei ausgeprägter pulmonaler Hypertonie - die bei der COPD selten vorkommt - scheidet die Einzellungentransplantation als Option wegen der Gefahr eines postoperativen schweren Reperfusionsschadens aus. Bei beidseitigen Bronchiektasen und Atemwegskolonisation mit Problemkeimen sollte ebenfalls keine Einzellungentransplantation erfolgen.

In Tab. 10 sind die Differenzialindikationen zur Durchführung einer LVR vs. einer LTx dargestellt.

\section{Palliative Therapie}

Angesichts des prinzipiell progressiven Krankheitsverlaufs und der damit in Verbindung stehenden progredienten klinischen Symptomatik einer leidvollen respiratorischen Insuffizienz können und müssen bei Patienten mit weit fortgeschrittener COPD auch primär palliativmedizinisch orientierte Therapieoptionen zum Einsatz kommen. Ergebnisgerichtete Studien zum Stellenwert von palliativmedizinischen Maßnahmen bei COPD liegen bislang nicht vor, dennoch darf nach Expertenmeinung erwartet werden, dass COPD Patienten im Endstadium von der Palliativmedizin profitieren.

Atemnot, Müdigkeit, Depressionen und Schmerzen unterschiedlicher Organzugehörigkeit sind die Hauptsymptome bei Patienten im letzten Jahr vor ihrem Tod [315]. Die Wirksamkeit von Sauerstoff zur Minderung der Atemnot bei COPD-Patienten im Endstadium ist durch kontrollierte Studien nicht belegt, als Expertenmeinung aber unbestritten [316]. Eine Metaanalyse zum Einsatz von Opioiden bei Dyspnoe jeder Ursache zeigt einen statistisch zu sichernden Effekt, der bei oraler oder parenteraler größer als bei inhalativer Applikation ist [317].

Bezüglich der palliativen Beeinflussung von Müdigkeit und rascher Erschöpfung werden Appetitstimulanzien, Zytokinantagonisten und Anabolika in Kombination mit physikalischer Therapie empfohlen (siehe auch Ernährung). Wenigstens bei Zweidrittel aller COPD-Patienten im Endstadium liegen Depression und Angststörungen vor. Zur Behandlung stehen in Abhängig- 
keit von der Qualität der Störung medikamentöse und nichtmedikamentöse Therapieverfahren zur Verfügung [318]. Die analgetische Therapie auch eines COPD-Patienten orientiert sich an den WHO-Empfehlungen [319].

Ausmaß und Zeitpunkt einer palliativen Therapie des kritisch kranken COPD-Patienten lassen sich heute noch nicht ausreichend sicher definieren. Ihre Bedeutung wird aber trotz weiterer Fortschritte der kausal ausgerichteten Basisbehandlung der COPD in den nächsten Jahren zweifelsfrei zunehmen.

\section{Management akuter Exazerbationen}

\section{Leitsätze}

Akute Exazerbationen bedürfen einer zusätzlichen medikamentösen Therapie. Hauptursachen sind Bronchialinfekte (Evidenzgrad B).

Medikamente der Wahl sind inhalative Bronchodilatatoren, insbesondere $\beta_{2}$-Sympathomimetika und/oder Anticholinergika und systemisch applizierte Glukokortikoide (Evidenzgrad A), bei unzureichendem Effekt auch Theophyllin (Evidenzgrad D).

COPD-Patienten mit Exazerbationen und den klinischen Zeichen eines bakteriellen Atemwegsinfektes können von einer Behandlung mit Antibiotika profitieren [320] (Evidenzgrad B).

Bei Patienten mit respiratorischer Partialinsuffizienz ist die Sauerstoffgabe indiziert, bei respiratorischer Insuffizienz mit Hyperkapnie im Rahmen der akuten Exazerbation der Einsatz der nichtinvasiven Beatmung mit positivem Druck (NIPPV) (Evidenzgrad A).

\section{Definition}

Eine COPD-Exazerbation ist als ein Ereignis im Verlauf der Erkrankung definiert, das durch eine Änderung der Dyspnoe, des Hustens und/oder des Auswurfs charakterisiert ist. Diese Änderung geht über die täglichen Schwankungen der Symptome hinaus, beginnt akut und macht eine Änderung der Medikation notwendig.

Während in manchen Publikationen bereits die Erhöhung der Dosis der Bronchodilatatoren als Ausdruck einer Exazerbation gewertet wird, fordern andere Autoren eine systemische Glukokortikoid- und/oder eine Antibiotikatherapie. In anderen Untersuchungen wird die Notwendigkeit einer Notarztbehandlung oder gar einer stationären Behandlung als Basis der Definition der Exazerbation gewählt. Wieder andere Definitionen orientieren sich an der Symptomatik. Diese unterschiedlichen Definitionen erschweren die vergleichende Bewertung von Studien erheblich.

Akute Verschlechterungen treten bei COPD-Patienten häufig auf [321 - 323]. Über die Prognose akuter Exazerbationen in Abhängigkeit vom Schweregrad liegen unterschiedliche Daten vor. Während ca. 50\% der meist leichten Exazerbationen ohne ärztlichen Kontakt von den betroffenen Patienten selbst bewältigt werden [48], liegt die Krankenhaussterblichkeit von COPD-Patienten mit schweren Exazerbationen zwischen 3\% und 10\% [324-328]. Noch schlechter ist die Prognose bei Aufnahme auf die Intensivstation. Die Sterblichkeit erreicht etwa 40\% innerhalb eines Jahres [325 - 328] und bei Patienten mit einem Alter von mehr als 65 Jahren sogar bis zu 59\% [328].

Häufigste Ursachen der Exazerbationen sind wahrscheinlich virale und/oder bakterielle Atemwegsinfektionen. In ca. 30\% der
Fälle kann die Ursache bislang nicht identifiziert werden [329-331].

Folgende Erreger werden als die häufigsten Verursacher einer Exazerbation angesehen:

H. influenzae,

S. pneumoniae,

M. catarrhalis,

Enterobakterien Species,

$P$. aeruginosa,

virale Erreger (Influenzaviren, Respiratory Syncitial Virus

(RSV), Rhino-, Corona- und Adenoviren).

Differenzialdiagnostisch abzugrenzen sind Pneumonien, Herzinsuffizienz, Pneumothorax, Pleuraergüsse, Lungenembolie, Arrhythmien oder ein Thoraxtrauma.

\section{Diagnostik}

Leitsymptom der akuten Exazerbation ist zunehmende Atemnot, häufig verbunden mit vermehrtem Husten, Zunahme von Menge und Viskosität des Sputums und/oder gelb-grüner Verfärbung des Auswurfs, Engegefühl im Brustraum und gelegentlich Fieber. Unspezifische Zeichen wie Bewusstseinstrübung bis hin zum Koma, Schlafstörungen, leichtere Ermüdbarkeit und Depressionen können hinzutreten.

Für eine schwere akute Exazerbation sprechen eine neu aufgetretene oder progrediente zentrale Zyanose, periphere Ödeme, der Einsatz der so genannten „Atemhilfsmuskulatur“ bei der Inspiration sowie eine hämodynamische Instabilität. Für die Einschätzung der Gefährdung des Patienten durch die Exazerbation wesentlich sind Kenntnisse über den Zustand des Patienten vor der Exazerbation, über Häufigkeit und Schweregrad früher durchgemachter Exazerbationen, über die bisherige Therapie und die Komorbiditäten. Wichtigstes diagnostisches Verfahren ist die Blutgasanalyse, die eine Einschätzung des Schweregrades der respiratorischen Insuffizienz sowie anhand des zusätzlich bestimmten Säure-Basen-Haushaltes auch der Gefährdung des Patienten erlaubt.

Eine respiratorische Insuffizienz liegt bei einem arteriellen Sauerstoffpartialdruck von weniger als $60 \mathrm{~mm} \mathrm{Hg}$ bzw. einer Sauerstoffsättigung von weniger als $90 \%$ bei Atmung von Raumluft vor.

Im Falle einer respiratorischen Globalinsuffizienz bei Sauerstoffpartialdrücken von < $50 \mathrm{~mm} \mathrm{Hg}, \mathrm{CO}_{2}$-Partialdrücken $>70 \mathrm{~mm} \mathrm{Hg}$ und $\mathrm{pH}$-Werten $<7,30$ muss von einer lebensbedrohlichen Situation mit der Notwendigkeit einer intensivmedizinischen Behandlung ausgegangen werden [332].

Kenngrößen der Ventilation sind im Rahmen einer akuten Exazerbation, insbesondere bei älteren Patienten, häufig nicht korrekt zu bestimmen.

Anhaltspunkte für eine schwere Exazerbation bieten Peak-FlowWerte unter $100 \mathrm{l} / \mathrm{min}$ und eine $\mathrm{FEV}_{1}<1 \mathrm{l}$ [333-335]. Wichtiger als die Absolutwerte sind akute Verschlechterungen wesentlicher Kenngrößen der Atemwegsobstruktion.

Zur Labordiagnostik gehören: BSG, Blutbild, CRP, D-Dimere, Elektrolyte, Kreatinin und Blutzucker.

Eine mikrobiologische Sputumuntersuchung (Gramfärbung und Bakterienkultur mit Resistenztestung) wird nur bei Patienten mit häufigen Exazerbationen ( $>3$ pro Jahr), Therapieversagen und/oder bei besonders schweren Erkrankungen mit Verdacht auf multiresistente Bakterien empfohlen. Voraussetzungen sind das Vorliegen eines makroskopisch purulenten Sputums und die Gewährleistung der notwendigen logistischen Voraussetzungen 


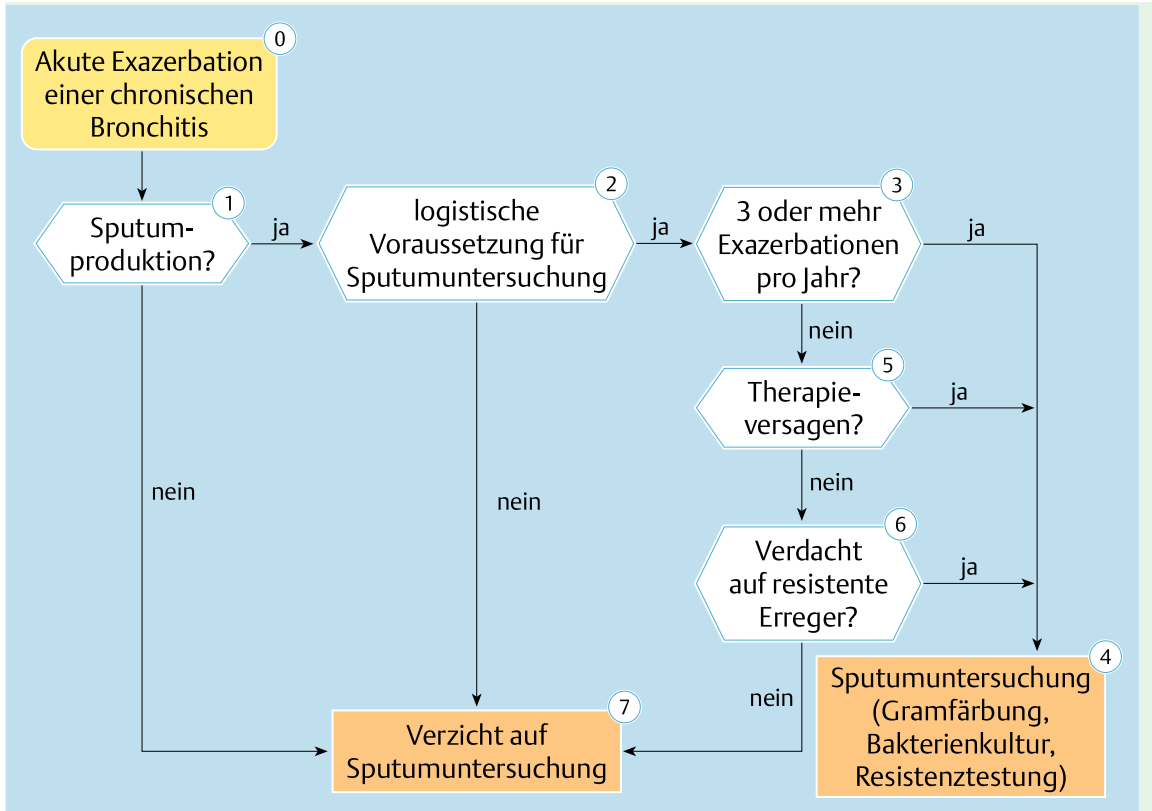

Abb. 5 Sputumdiagnostik bei Patienten mit akuter Exazerbation der COPD.

\section{Stationäre Behandlung}

schwere Atemnot

schlechter Allgemeinzustand

rasche Progression der Symptomatik

\section{Aufnahme auf die Intensivstation}

schwere Atemnot mit fehlendem Ansprechen auf die initiale Notfalltherapie

komatöser Zustand

persistierende arterielle Hypoxämie $\left(\mathrm{PaO}_{2}<50 \mathrm{~mm} \mathrm{Hg}\right)$ trotz Sauerstoffgabe und/oder schwere oder progrediente Hyperkapnie $\left(\mathrm{PaCO}_{2}>70 \mathrm{~mm} \mathrm{Hg}\right)$ und/oder schwere/zunehmende respiratorische Azidose $(\mathrm{pH}$ $<7,30$ ) trotz nichtinvasiver Beatmung mit positivem Druck (NIPPV)

\section{Bewusstseinstrübung}

Auftreten/Zunahme von Ödemen und/oder einer

zentralen Zyanose

fehlendes Ansprechen auf die initiale Therapie

unklare Diagnose

neu aufgetretene Arrhythmien

bedeutsame Komorbidität

höheres Lebensalter

unzureichende häusliche Betreuung

(Transport und Verarbeitung innerhalb von 2-4 Stunden) (O Abb. 5).

Bei jeder Sputumanalyse sollte auch eine mikroskopische Untersuchung mit Auszählung von Plattenepithelzellen und Leukozyten erfolgen, um darüber auf die Qualität des Sputums schließen zu können.

Zur differenzialdiagnostischen Abklärung bei akuter Atemnot sind eine Röntgenaufnahme der Thoraxorgane (möglichst in 2 Ebenen) sowie ein EKG sinnvoll. Darüber hinaus sollte bei unklarer Ursache der Atemnot ein D-Dimer-Test erfolgen. Bei pathologischem Ausfall ist eine Lungenarterienembolie auszuschließen [336].

In Abhängigkeit vom Schweregrad der Grunderkrankung, den Folgen der akuten Exazerbation und der Komorbidität kommen sowohl eine ambulante als auch eine stationäre Behandlung in Betracht. Kriterien für die Krankenhauseinweisung sowie für die Aufnahme auf die Intensivstation sind $\bigcirc$ Tab. $11 \mathrm{zu}$ entnehmen. Respiratorische Azidose, eine notwendige Beatmung und bedeutsame Komorbiditäten sind Prädiktoren einer ungünstigen Prognose [324].

\section{Therapie der Exazerbation}

Das Management der akuten Exazerbationen sollte in Abhängigkeit vom Schweregrad der Exazerbation und der Beeinträchtigung des Patienten ambulant oder stationär durchgeführt werden ( Abb.6). Tab. 12 enthält eine Zuordnung der medikamentösen und apparativen Therapieoptionen zu drei anhand klinischer Kriterien festgelegter Schweregrade der Exazerbation.

Die leichtgradige Exazerbation ist durch eine leichte subjektive Beeinträchtigung mit oder ohne leichte Verschlechterung der Lungenfunktion mit einer Abnahme der $\mathrm{FEV}_{1}$ von maximal $20 \%$ des Ausgangswertes vor Beginn der Exazerbation gekennzeichnet. Patienten mit leichtgradiger Exazerbation können ambulant behandelt werden. Als medikamentöse Therapieoptionen stehen in erster Linie $\beta_{2}$-Sympathomimetika und/oder Anticholinergika zur Verfügung, wobei für die langwirksamen Substan- 


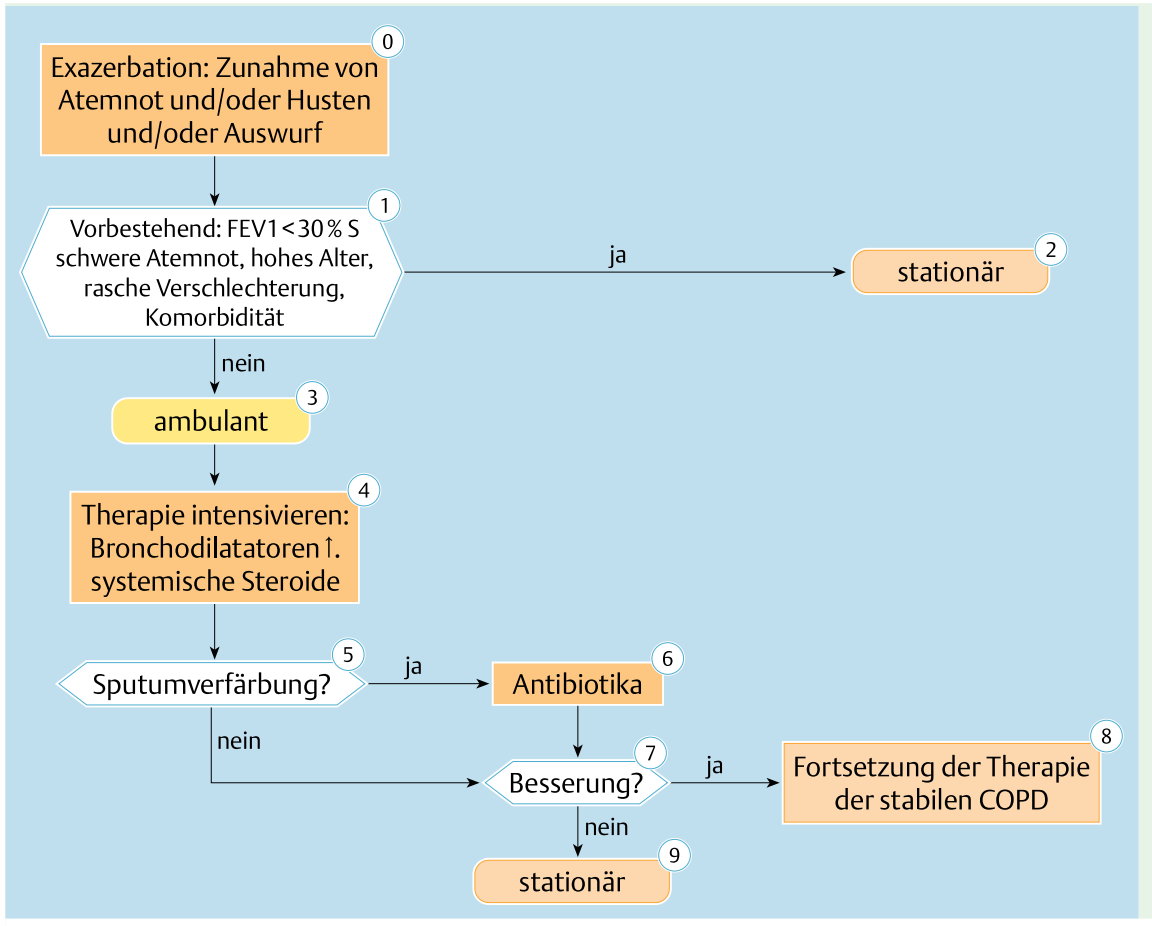

Abb. 6 „Management der Exazerbation der COPD“.

\begin{tabular}{ll}
$\begin{array}{l}\text { Schweregrad } \\
\text { alle Schweregrade }\end{array}$ & Kennzeichen \\
leichtgradig & $\begin{array}{l}\text { leichte subjektive Verschlechterung } \pm \text { Ver- } \\
\text { schlechterung der Lungenfunktion }\end{array}$ \\
\hline mittelgradig & $\begin{array}{l}\text { Atemnot } \uparrow / \text { Husten } \uparrow \\
+ \text { Verschlechterung der Lungenfunktion }\end{array}$ \\
schwergradig & $\begin{array}{l}\text { Bewusstseinstrübung } \\
\text { Tachykardie/-pnoe } \\
\text { Zyanose (neu/progredient) } \\
\text { Ödeme }\end{array}$
\end{tabular}

zen beider Medikamentengruppen noch keine Evidenz für den Therapieeinsatz zur Bewältigung der Exazerbation vorliegt.

Die mittelgradige Exazerbation ist durch eine stärkere Verschlechterung des Befindens mit zunehmender Atemnot und/ oder Husten gekennzeichnet sowie durch eine messbare Einschränkung der Lungenfunktion.

Zusätzlich zu Anticholinergika und/oder $\beta_{2}$-Sympathomimetika können systemische Glukokortikosteroide (Evidenzgrad A) und bei fehlender Besserung auch Theophyllin eingesetzt werden (Evidenzgrad D). Auch die mittelgradige Exazerbation kann überwiegend ambulant behandelt werden.

Kennzeichen der schweren Exazerbation sind das Auftreten von Ödemen, eine neu aufgetretene oder progrediente Zyanose, Bewusstseinstrübungen bis zu komatösen Zuständen sowie das Auftreten von Tachykardien, Arrhythmien und Tachypnoe. Die schwere Exazerbation sollte stationär behandelt werden. Als Therapieoptionen stehen neben Anticholinergika, $\beta_{2}$-Sympathomimetika, systemischen Glukokortikosteroiden und Theophyllin die Behandlung mit Sauerstoff, die nichtinvasive Beatmung sowie die Therapie der Komplikationen zur Verfügung.

Die Therapie aller Schweregrade sollte den Verzicht auf Tabakkonsum, den Einsatz von Antibiotika bei purulentem Sputum und die Behandlung der Komorbiditäten einschließen.

Therapie
Nikotinverzicht
Antibiotika bei purulentem Sputum
Therapie der Komorbidität(en)
Anticholinergika u./o.
$\beta_{2}$-Sympathomimetika
evtl.
+ systemische Glukokortikoide
+ Theophyllin
+ Sauerstoff
\pm NIV
\pm Therapie der Komplikationen

Tab. 12 Schweregradorientierte Behandlung der Exazerbation der COPD

\section{Medikamentöse Therapie}

Bronchodilatatoren. Kurzwirksame $\quad \beta_{2}$-Sympathomimetika (initial 100-200 $\mu$ g eines Dosier-Aerosols bzw. Pulverinhalators) sind die bevorzugten Bronchodilatatoren in der Therapie der akuten Exazerbation der COPD [5,6,8] (Evidenzgrad A). Bei unzureichender Besserung können zusätzlich Anticholinergika höher dosiert verabreicht werden mit initial $250-500 \mu \mathrm{g}$, z. B. über Vernebler, schließlich Theophyllin i.v. mit einer Initialdosis von $200 \mathrm{mg}$, oder einer kontinuierlichen Infusion mit $0,5 \mathrm{mg} / \mathrm{kg} /$ Stunde, falls der Patient nicht mit Theophyllin vorbehandelt ist. Der Stellenwert der Theophyllintherapie ist umstritten [337-341]. Einige Studien zeigen eine Verschlechterung des Gasaustausches und der arteriellen Hypoxämie bei Besserung von Obstruktion und Lungenüberblähung $[338,339]$. Die aktuellste Studie [342] ergab, dass die Gabe von Theophyllin vs. Plazebo zusätzlich zu hoch dosierten Bronchodilatatoren und systemischen Glukokortikoiden keinen klinisch bedeutsamen Effekt hat. Lediglich eine geringfügige Verbesserung des Säure-BasenHaushalts war in der Theophyllingruppe feststellbar. Um unerwünschte Effekte des Theophyllins zu vermeiden, sind die Theophyllin-Serumspiegel engmaschig zu kontrollieren [340,341]. Antibiotika Antibiotika sind nur bei Exazerbationen infolge eines bakteriellen Atemwegsinfektes wirksam. Bakterielle Exazer- 
bationen sind neben einer Zunahme von Dyspnoe, Husten und Sputummenge durch das Auftreten eines purulenten Sputums $[320,329]$ charakterisiert.

Procalcitonin III, ein spezifischer Marker für bakterielle Infektionen, könnte ein Marker für die Behandlungsbedürftigkeit von Exazerbationen mit Antibiotika sein [343] (Evidenzgrad B), allerdings ist der Marker aus Kostengründen bisher nicht im klinischen Alltag etabliert.

Eine Antibiotikatherapie wird empfohlen, wenn alle klinischen Zeichen (Dyspnoe, vermehrtes und gelbgrün verfärbtes Sputum) der bakteriellen Infektion vorliegen (Evidenzgrad B, Empfehlungsgrad A), bei allen Patienten mit sehr schwerer COPD (Evidenzgrad D) und bei Patienten, die eine respiratorische Unterstützung mittels nichtinvasiver oder invasiver maschineller Beatmung benötigen [344] (Evidenzgrad A).

Eine Antibiotikatherapie kann erwogen werden (Evidenzgrad D) bei Patienten aller Schweregrade mit häufig rezidivierenden akuten Exazerbationen (> 4/Jahr) und Patienten aller Schweregrade mit relevanter kardialer Komorbidität.

Die Wahl des Antibiotikums für die kalkulierte Therapie der Exazerbation entsprechend den oben genannten Indikationen richtet sich nach der Schwere der Lungenfunktionseinschränkung und dem Risiko für eine kausale Beteiligung von Pseudomonasspecies an der Exazerbation. Für Patienten mit einer Erkrankung im Stadium I und II werden in Abhängigkeit von der lokalen Resistenzlage in erster Linie Aminopenicilline (ggf. plus Betalactamase-Inhibitoren) empfohlen [345]. Makrolide sollten aufgrund der aktuellen deutschen Resistenzsituation nur in Ausnahmefällen (z. B. Penicillinunverträglichkeit) zum Einsatz kommen [346] (Evidenzgrad B).

In unkomplizierten Fällen können auch Tetrazykline und Oralcephalosporine eingesetzt werden [345]. Bei fehlendem Ansprechen auf andere Antibiotika kann auch der Einsatz von Ketoliden erwogen werden (Evidenzgrad B).

Für Patienten im Stadium III und IV werden Aminopenicilline mit Betalaktamaseinhibitor (Amoxicillin + Clavulansäure oder Sultamicillin) oder Fluorchinolone mit Pneumokokkenwirksamkeit (Levofloxacin, Moxifloxacin) empfohlen.

Ein Risiko für einen kausalen Einfluss von Pseudomonas aeruginosa besteht bei Vorliegen folgender Faktoren [347] (Evidenzgrad B):

pulmonale Komorbidität (strukturelle chronische Erkran-

kungen wie COPD im Stadium IV, Bronchiektasen, Mukovis-

zidose),

stationärer Aufenthalt von mehr als 2 Tagen in den letzten 30 Tagen vor Beginn der akuten Pneumonieepisode,

Glukokortikoidtherapie (mindestens $10 \mathrm{mg}$ Prednisonäqui-

valent über mindestens 4 Wochen),

Aspiration,

Breitspektrum-Antibiotikatherapie über mehr als 7 Tage innerhalb des letzten Monats,

Malnutrition.

Bei Patienten mit einem solchen Risiko müssen pseudomonaswirksame Substanzen eingesetzt werden, wobei Cipro- und Levofloxacin die einzigen oral verfügbaren Substanzen darstellen. Eine intravenöse Therapie wird nur empfohlen, wenn der Patient nicht schlucken kann, unsichere Resorptionsverhältnisse bestehen, eine schwere akute Exazerbation oder ein Pseudomonasrisiko vorliegen.

Die Dauer der Antibiotikatherapie einer akuten bakteriellen Exazerbation liegt in der Regel bei 5-10 Tagen. Kriterien des Ansprechens der antibiotischen Therapie bei Exazerbation sind nur unzureichend definiert. Zeichen des klinischen (symptombezogenen) Ansprechens sind der Rückgang der Dyspnoe und der Sputummenge und die Entfärbung des Sputums.

Ein signifikanter Anteil von Patienten weist nur eine unzureichende Besserung der Exazerbationssymptome auf. Das therapeutische Vorgehen bei diesen Patienten ist nicht etabliert. In jedem Fall sollte eine protrahierte Gabe antimikrobieller Substanzen (über 10 Tage) vermieden werden, da die Wahrscheinlichkeit einer Resistenzzunahme nach diesem Zeitraum ansteigt.

Ein Therapieversagen äußert sich in einer persistierenden Symptomatik trotz adäquater Therapie von mindestens 48- 72 h Dauer. Zum Vorgehen im Rahmen eines Therapieversagens liegen keine Studien vor.

Bei Patienten mit Therapieversagen ist die antibiotische Medikation abzusetzen und nach einer Behandlungspause von 2 - 3 Tagen eine mikrobiologische Diagnostik durchzuführen.

Glukokortikoide Glukokortikoide sollen oral oder intravenös zusätzlich zur Gabe von Bronchodilatatoren eingesetzt werden. Sie verkürzen die Genesungszeit und führen zu einer rascheren Besserung der Lungenfunktion [348-350] (Evidenzgrad A). Sie sollten bei einer Einschränkung der $\mathrm{FEV}_{1}<50 \%$ des Sollwertes im Rahmen einer Exazerbation zusätzlich zu Bronchodilatatoren gegeben werden (Empfehlungsgrad A). Eine Dosis von 20-40 mg Prednisolonäquivalent über 10-14 Tage wird empfohlen [348,349] (Evidenzgrad C). Eine längere Behandlungsdauer führt nicht zu einer höheren Effizienz, erhöht aber das Risiko von unerwünschten Effekten [350].

Falls im Rahmen der ambulanten Therapie eine Wiederherstellung des Ausgangszustandes vor der Exazerbation nicht gelingt bzw. die Exazerbation progredient verläuft oder weitere Maßnahmen wie Sauerstoffgabe bzw. eine nicht invasive Beatmung erforderlich werden, ist eine stationäre Einweisung notwendig, die primär dann erfolgen sollte, wenn die Kriterien in 0 Tab. 11 erfüllt sind.

\section{Zusätzliche Maßnahmen bei der Behandlung akuter Exa-} zerbationen im Krankenhaus

Im Krankenhaus wird der Schweregrad der Exazerbation anhand der Symptome, der arteriellen Blutgase und des Röntgenbildes der Thoraxorgane bestimmt. Zusätzlich müssen die Suche nach den Ursachen der Exazerbation und die Diagnostik etwaiger Begleitkrankheiten unter Einschluss von EKG-Registrierung und Laborstatus rasch erfolgen.

Bei respiratorischer Insuffizienz ist die Sauerstoffgabe über eine Nasensonde bzw. Atemmaske sofort einzuleiten ( Abb. 7). Falls die in Tab. 11 genannten Kriterien zutreffen, sollte der Patient auf die Intensivstation verlegt werden.

Ziel der Sauerstofftherapie ist eine adäquate Oxygenierung mit arteriellen $\mathrm{PaO}_{2}$-Werten von mehr als $60 \mathrm{~mm} \mathrm{Hg}$ bzw. einer Sauerstoffsättigung von mehr als $90 \%$. Eine Kontrolle zur Beurteilung des Erfolges und zur Überprüfung einer unter Sauerstoffgabe auftretenden $\mathrm{CO}_{2}$-Retention kann bereits nach 20 Minuten durchgeführt und sollte im Verlauf überprüft werden.

Unter stationären Bedingungen mit Kontrolle der Herzfrequenz können kurzwirksame $\beta_{2}$-Sympathomimetika höher dosiert und auch parenteral appliziert werden, bei fehlender Besserung mit Anticholinergika und ggf. auch mit Theophyllin kombiniert werden.

Diuretika sind bei peripheren Ödemen und erhöhtem Jugularvenendruck indiziert. Für die Initialtherapie ist die Gabe von $40 \mathrm{mg}$ Furosemid i.v. empfehlenswert. Die Flüssigkeitsbilanz ist sorgfältig zu überwachen. Eine leicht zu trinkende orale Zusatz- 


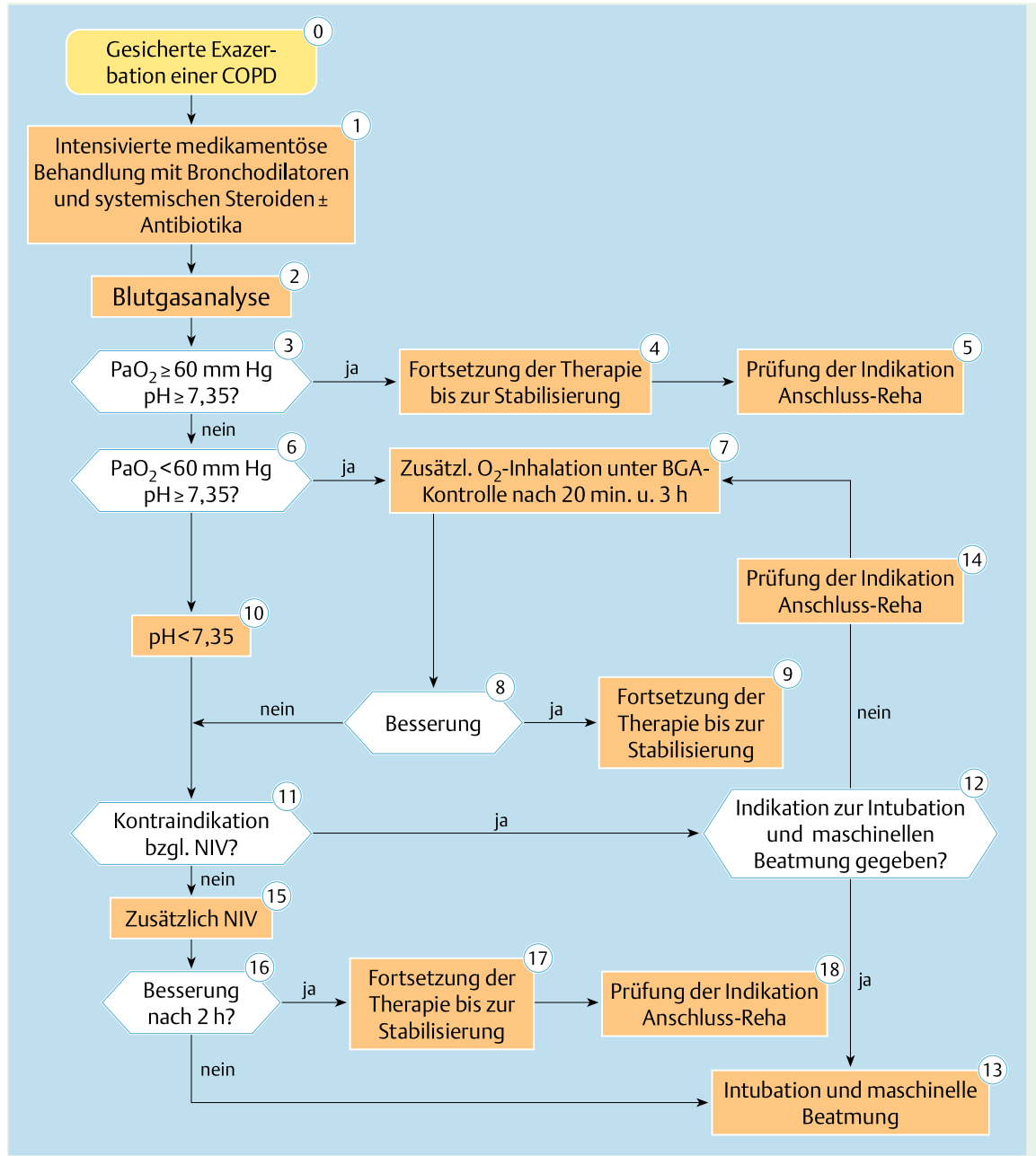

Abb. 7 Stationäre Therapie der Exazerbation der COPD.

ernährung oder gegebenenfalls auch eine intravenöse Kalorienzufuhr können notwendig werden, wenn der Patient infolge ausgeprägter Atemnot keine Nahrung zu sich nimmt. Eine Alternative bietet die Ernährung über eine Magensonde. Bei immobilisierten Patienten, bei Patienten mit Polyglobulie, Dehydratation, bei Zeichen der chronisch venösen Insuffizienz und/oder thromboembolischen Erkrankungen sollte eine Heparinisierung erfolgen, wobei niedermolekulare Heparine wie Standardheparine einsetzbar sind.

Die Bedeutung der Physiotherapie bei Patienten mit akuter Exazerbation unter Krankenhausbedingungen ist nicht eindeutig geklärt [351]. Bei großen Sputummengen und lobären Atelektasen sind Lagerungsdrainagen empfehlenswert. Hierbei ist aber auch eine Bronchoskopie in Erwägung zu ziehen, insbesondere wenn die Exazerbation einen hohen Schweregrad aufweist. Die Sekretelimination kann durch Wärmeapplikation sowie den Einsatz von Hilfsmitteln mit und ohne Oszillationen und nachfolgendem Huffing, ggf. Absaugen, unterstützt werden.

\section{Beatmung}

Mithilfe einer nichtinvasiven oder invasiven Beatmung können die im Rahmen schwerer Exazerbationen häufig überlastete Atemmuskulatur [352,353] bis zur Beseitigung der Ursache der Exazerbation entlastet und hierdurch Morbidität und Mortalität reduziert werden. Bei akuten Exazerbationen der COPD kann die Beatmung nichtinvasiv über eine Gesichts- oder Nasenmaske oder invasiv über eine orotracheale Intubation im Sinne einer konventionellen invasiven Beatmung erfolgen.
Nichtinvasive Beatmung mit positivem Druck (NIPPV) Die nichtinvasive Beatmung mit positivem Druck wurde bei akuter respiratorischer Insuffizienz von COPD-Patienten in den letzten Jahren in zahlreichen unkontrollierten und 5 randomisierten kontrollierten Untersuchungen geprüft [354-358]. Die NIPPV führte zu einer Reduktion der respiratorischen Azidose, einem Abfall des $\mathrm{PaCO}_{2}$ sowie einer Abnahme der Atemnot in den ersten 2 - 4 Stunden nach Beginn der Therapie sowie auch zu einer Senkung der Mortalität [354-356]. Eine Intubation mit invasiver Beatmung konnte häufig verhindert werden und wurde in nur 15-20\% der Fälle notwendig [357-359]. Im Vergleich zur invasiven pressure support ventilation verkürzt die nichtinvasive Beatmung mit positivem Druck (NIPPV) die Entwöhnungszeit, reduziert den Aufenthalt auf der Intensivstation, führt zu einer Reduktion der Häufigkeit nosokomialer Pneumonien und verbessert die Prognose während eines Beobachtungszeitraums von 60 Tagen [359] (Evidenzgrad A). Die nichtinvasive Beatmung ist daher, falls keine Kontraindikationen bestehen, der invasiven Beatmung vorzuziehen (Empfehlungsgrad A).

Die Ein- und Ausschlusskriterien für die NIPPV sind in $\bigcirc$ Tab. 13 dargestellt.

Wenn die in Tab. 13 genannten Ausschlusskriterien zutreffen, sollte die Indikation zu einer invasiven Beatmung gestellt werden ( Tab. 14).

Invasive Beatmung Der Nutzen der invasiven Beatmung bei COPD-Patienten im Endstadium ist Gegenstand zahlreicher Diskussionen. Patienten ohne wesentliche Komorbiditäten, mit potenzieller Reversibilität der Ursache der akuten Exazerbation 
Einschlusskriterien

Schwere Atemnot mit Einsatz der „Atemhilfsmuskulatur“ und paradoxer abdominaler Atmung Azidose $(\mathrm{pH}<7,35)$ und Hyperkapnie (mit $\mathrm{PaCO}_{2}>50 \mathrm{~mm} \mathrm{Hg}$ ) Atemfrequenz $>25 / \mathrm{min}$

\section{Ausschlusskriterien}

Atemstillstand

Herz-Kreislauf-Instabilität (Herzinfarkt, kardiogener Schock, Hypotonie, schwere Arrhythmie)

Bewusstseinstrübung (Somnolenz $\rightarrow$ Koma)

Fehlende Kooperation des Patienten

erhöhte Gefahr von Regurgitation und Aspiration (Schluckstörung, Ileus, gastrointestinale Blutung, kürzliche Operation im Bereich von Gesicht, Speiseröhre und Oberbauch, Gesichtstrauma, Fehlbildungen im Nasopharynx)

visköses Sekret, große Sputummenge

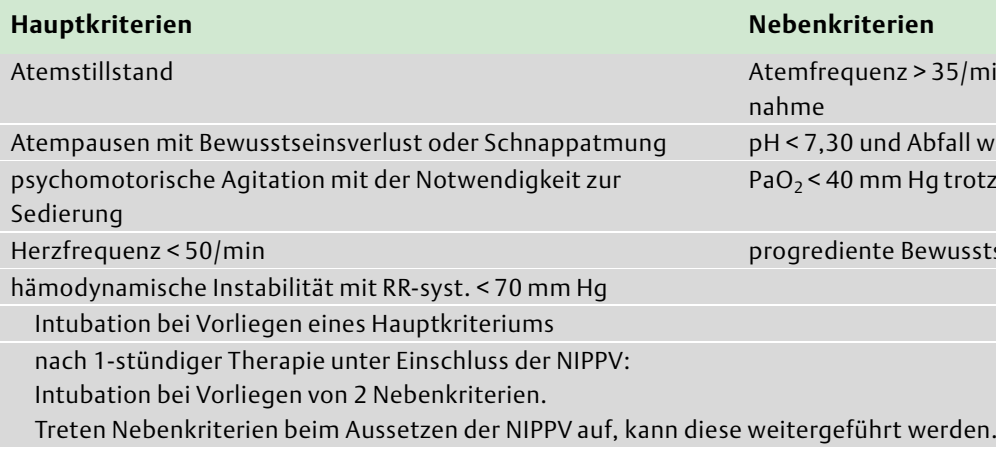

Tab. 13 Ein- und Ausschluss kriterien für die nichtinvasive Beatmung (NIPPV) bei COPDPatienten mit respiratorischer Insuffizienz
(Infektion) sowie relativ mobile Patienten profitieren am häufigsten von einer derartigen Therapie. An Komplikationen sind die ventilatorassoziierte Pneumonie, das Barotrauma und Entwöhnungsprobleme zu nennen. Bei invasiv beatmeten COPDPatienten ist mit einer deutlichen Verlängerung des Aufenthaltes auf der Intensivstation zu rechnen. Die Hospitalsterblichkeit der maschinell beatmeten Patienten liegt etwa bei $17-30 \%$. Bei der Entscheidung zur invasiven Beatmung ist der zuvor festgelegte Wille des Patienten hinsichtlich einer derartigen Therapiemaßnahme zu berücksichtigen.

\section{Management nach der akuten Exazerbation im Kran- kenhaus}

Die Krankenhausverweildauer für eine Exazerbation von COPDPatienten muss individuell festgelegt werden. Mögliche Entlassungskriterien sind:

die Fähigkeit des Patienten, auf ebener Strecke zu gehen, wenn dies vor der Exazerbation möglich war; ferner zu essen und zu schlafen ohne häufige Unterbrechungen infolge Dyspnoe,

Stabilität der Symptomatik und der arteriellen Blutgase über mindestens 24 Stunden,

die Reduktion der bedarfsorientiert eingesetzten inhalativen kurzwirksamen $\beta_{2}$-Sympathomimetika auf weniger als $6 \mathrm{x}$ am Tage.

Vor der Entlassung sollten der Patient und/oder seine Angehörigen den Einsatz der für die Therapie der Erkrankung notwendigen Medikation kennen, die Inhalationstechniken beherrschen und gegebenenfalls über die weitere Durchführung der LOT und/ oder NIPPV-Therapie informiert sein. Außerdem ist zu prüfen, ob der Patient einer Anschlussrehabilitation zuzuleiten ist, um erneuten Exazerbationen mit Krankenhausaufenthalten vorzubeugen.

Die weitere Versorgung muss in Zusammenarbeit mit dem Patienten, seinen Angehörigen, dem Hausarzt und dem Pneumolo- gen gesichert sein. 4-6 Wochen nach der Entlassung sollte eine Überprüfung des Gesundheitszustandes mit Messung der Lungenfunktion, Kontrolle der Inhalationstechnik und der Kenntnisse über die notwendige Behandlung erfolgen.

Außerdem sollten die Meidung von Risikofaktoren, insbesondere der Verzicht auf Nikotinkonsum, die Notwendigkeit für eine LOT und/oder eine Heimbeatmung überprüft werden.

Der Patient muss eine korrekte Inhalationstechnik beherrschen sowie die Fähigkeit zur Erkennung einer Exazerbation [148,360] und zur Einleitung von Selbsthilfemaßnahmen (Patientenschulung, Rehabilitation) erwerben.

\section{Exazerbationsprophylaxe}

Exazerbationen treten bevorzugt im Winter auf. Der Häufigkeitsgipfel liegt in den ersten 2 - 3 Monaten eines Jahres. In dieser Zeit sind Krankenhauseinweisungen wegen einer akuten Exazerbation bis zu 6-mal häufiger als in den Sommermonaten. Mit fortschreitendem Schweregrad der COPD nehmen das Exazerbationsrisiko und die Häufigkeit der stationären Behandlungen zu. Prädiktoren für ein erhöhtes Hospitalisierungsrisiko sind vorausgegangene Hospitalisierungen wegen Exazerbation chronische Mukushypersekretion, chronische respiratorische Globalinsuffizienz, chronisches Cor pulmonale sowie hohes Lebensalter in Verbindung mit Multimorbidität [324]. Angesichts der gesteigerten Morbidität, der geminderten Lebensqualität und des erhöhten Mortalitätsrisikos ist - auch aus ökonomischen Gründen - die Prävention der akuten Exazerbation ein wichtiges medizinisches Ziel.

Empfohlene Maßnahmen zur Exazerbationsprophylaxe sind:

Raucherentwöhnung (Evidenzgrad A),

Meidung von inhalativen Noxen (Arbeitsplatz, Hobby) und Kälte,

Schutzimpfungen (Influenza: Evidenzgrad A; Pneumokokken: Evidenzgrad D),

inhalative Anticholinergika [51,52,361] (Evidenzgrad A), 


\begin{tabular}{|c|c|}
\hline BK-Nr. 4301 & $\begin{array}{l}\text { „Durch allergisierende Stoffe verursachte obstruktive Atemwegserkrankungen (einschließlich } \\
\text { Rhinopathie), die zur Unterlassung aller Tätigkeiten gezwungen haben, die für die Entstehung, } \\
\text { die Verschlimmerung oder das Wiederaufleben der Krankheit ursächlich waren oder sein kön- } \\
\text { nen“ }\end{array}$ \\
\hline BK-Nr. 4302 & $\begin{array}{l}\text { „Durch chemisch-irritativ oder toxisch wirkende Stoffe verursachte obstruktive Atemwegser- } \\
\text { krankungen, die zur Unterlassung aller Tätigkeiten gezwungen haben, die für die Entstehung, } \\
\text { die Verschlimmerung oder das Wiederaufleben der Krankheit ursächlich waren oder sein kön- } \\
\text { nen“ }\end{array}$ \\
\hline BK-Nr. 1315 & $\begin{array}{l}\text { „Erkrankungen durch Isocyanate, die zur Unterlassung aller Tätigkeiten gezwungen haben, die } \\
\text { für die Entstehung, die Verschlimmerung oder das Wiederaufleben der Krankheit ursächlich } \\
\text { waren oder sein können“ }\end{array}$ \\
\hline BK-Nr. 4111 & $\begin{array}{l}\text { "Chronische obstruktive Bronchitis oder Emphysem von Bergleuten unter Tage im Steinkoh- } \\
\text { lenbergbau bei Nachweis der Einwirkung einer kumulativen Dosis von in der Regel } 100 \text { Fein- } \\
\text { staubjahren }\left[\left(\mathrm{mg} / \mathrm{m}^{3}\right) \times \text { Jahre]“" }\right.\end{array}$ \\
\hline
\end{tabular}

Tab. 15 Obstruktive Atemwegserkrankungen nach der Liste der Berufskrankheiten in der Fassung der Berufskrankheiten-Verordnung (BKV) vom 31.10 .1997 inhalative Glukokortikoide [54,120,168,169] (Evidenzgrad A), langwirksame $\beta_{2}$-Sympathomimetika [54,125,168,169] (Evidenzgrad $A$ ),

Patientenschulung (Evidenzgrad B).

\section{Appendix A}

\section{Berufsbedingte Bronchitis}

Im angloamerikanischen Sprachraum ist die „berufsbedingte Bronchitis“ (occupational bronchitis) ein feststehender Begriff und wird als Folge einer Exposition gegenüber irritativ wirkenden Stäuben und Gasen am Arbeitsplatz angesehen.

Als gefährdend werden unter anderem Bergbautätigkeiten, $\mathrm{Ar}$ beiten mit Rohbaumwolle und in der Getreideverladung, Schweiß-, Koksofen-, Isolier- und Feuerlöscharbeiten genannt, als Noxen quarzhaltige Stäube, Baumwollstäube, Getreidestäube, Schweißrauche, Mineralfasern und irritativ wirksame Gase wie Ozon, Stickstoffdioxid und Chlorgas [362]. Die beruflich verursachte Bronchitis wird in der Regel als warnender Hinweis auf eine vermehrte Exposition gegenüber Irritantien des Atemtraktes anzusehen sein.

\section{Bronchitis im Unfall- und Berufskrankheitenrecht}

Die alleinige Bronchitis ohne obstruktive Lungenfunktionseinschränkung erfüllt in Deutschland nicht die unfallversicherungsrechtlichen Voraussetzungen zur Anerkennung einer Berufskrankheit nach §9 Abs. 1 oder 2 des SGB 7. Dies liegt im Wesentlichen darin begründet, dass es für die nicht obstruktive Form der Bronchitis in arbeitsmedizinisch-epidemiologischen Studien bislang nicht ausreichend gelungen ist, ein Verdoppelungsrisiko zu belegen. Die Anerkennung einer obstruktiven Atemwegserkrankung als Berufskrankheit ist im Wesentlichen unter den BK-Nummern 4301, 4302, 1315 und 4111 möglich, wobei unter den erstgenannten drei Nummern die asthmatischen gegenüber den bronchitischen Erkrankungen im Vordergrund stehen [363]. Nach epidemiologischen Studien werden bei Beschäftigungen mit langjähriger Untertage-Tätigkeit im Steinkohlenbergbau Erkrankungen an chronisch obstruktiver Bronchitis und Lungenemphysem signifikant gehäuft angetroffen. Dies trifft auch zu, wenn radiologische Zeichen einer eindeutigen Silikose nicht vorliegen. Es gelang bei dieser Personengruppe, eindeutige Dosis-Wirkungs-Beziehung zwischen Einatmen der Staubmenge und der Häufigkeit des Auftretens von chronisch obstruktiver Bronchitis und Lungenemphysem nachzuweisen [364].
Zur Prüfung des Vorliegens der entsprechenden Berufskrankheit $4111[364,365]$ bedarf es der Errechnung der kumulativen Feinstaubdosis.

Die Dokumentation der Staubexposition im deutschen Steinkohlenbergbau ist seit den 60er Jahren praktisch lückenlos, so dass im Einzelfall nachvollziehbare Abschätzungen der kumulativen Feinstaubdosis möglich sind.

Die kumulative Feinstaubdosis ergibt sich aus der Feinstaubkonzentration in der Luft am Arbeitsplatz in $\mathrm{mg} / \mathrm{m}^{3}$ multipliziert mit der Anzahl der Expositionsjahre, bezogen auf jährlich 220 gefahrene Schichten zu je 8 Stunden Dauer.

Weiterhin kann eine Bronchitis Begleiterkrankung einer Pneumokoniose sein, also einer Lungenveränderung durch eingeatmeten (quarzhaltigen) Staub. Sofern die entsprechenden unfallversicherungsrechtlichen Voraussetzungen gegeben sind, sind die funktionsanalytisch nachweisbaren obstruktiven Folgezustände unter der jeweils für die Substanz zutreffenden BKNummer zu entschädigen. Tab. 15 enthält die obstruktiven Atemwegserkrankungen in der Fassung der BerufskrankheitenVerordnung vom 31.10.1997, ○ Tab. 16 mögliche Auslöser von Erkrankungen der Atemwege und Lunge, bei denen obstruktive Ventilationsstörungen vorkommen können.

Eine Bronchitis kann Folge eines Arbeitsunfalles sein, z.B. nach lokalisierten Entzündungen, Kontusion, Verletzung der großen Atemwege, Inhalationsintoxikation oder nach unfallbedingten neurologischen Erkrankungen.

Sofern haftungsbegründende und haftungsausfüllende Kausalität aus unfallversicherungsrechtlicher und gutachterlicher Sicht bejaht werden und ggf. die gefährdende Tätigkeit aufgegeben ist (BK 4302, 1315), ist die Minderung der Erwerbsfähigkeit (MdE) vom Gutachter einzuschätzen. Diese richtet sich nach anamnestischen, klinischen und funktionsanalytischen Kenngrößen. Von wesentlicher Bedeutung ist die Längsschnittbeurteilung der Erkrankung, d. h. es ist in aller Regel erforderlich, auf früher erstellte Originalbefunde zurückzugreifen. Ein Vorschlag zur gutachterlichen Wertung findet sich bei Kroidl u. Mitarb. [366].

\section{Bronchitis im sozialen Entschädigungsrecht und im Schwerbehindertenrecht}

Hinsichtlich des gutachterlichen Vorgehens im sozialen Entschädigungsrecht und im Schwerbehindertenrecht sei auf die Anhaltspunkte des Bundesministeriums für Arbeit- und Sozialordnung verwiesen [367].

\section{Prävention am Arbeitsplatz}

Als allgemeinen Staubgrenzwert hat die Senatskommission zur Prüfung gesundheitsschädlicher Arbeitsstoffe der Deutschen Forschungsgemeinschaft eine Konzentration des alveolengängi- 
Tab. 16 Mögliche Auslöser von Erkrankungen der Atemwege und Lunge, bei denen im weiteren Sinne obstruktive Ventilationsstörungen vorkommen können

\begin{tabular}{|l|l|}
\hline Auslöser & BK-Nummer \\
\hline Chrom & 1103 \\
\hline Vanadium & 1107 \\
\hline Fluor & 1308 \\
\hline Quarz & 4101,4102 \\
\hline Asbest & 4103 \\
\hline Aluminium & 4106 \\
\hline Nickel & 4109 \\
\hline Steinkohlengrubenstäube unter Tage & 4111 \\
\hline Verschimmeltes Heu, Stroh, Pilze & 4201 \\
\hline Rohbaumwolle, - Flachs, - - Hanf & 4202 \\
\hline
\end{tabular}

gen Anteils (bisher „Feinstaub“) von $1,5 \mathrm{mg} / \mathrm{m}^{3}$ und eine Konzentration des einatembaren Anteils (bisher „Gesamtstaub“) von $4 \mathrm{mg} / \mathrm{m}^{3}$ festgesetzt.

Überschreitungen sind zulässig, wobei die Höhe der zulässigen Überschreitungen das Zweifache des genannten allgemeinen Staubgrenzwertes nicht übertreffen sollte.

Nach gegenwärtigem wissenschaftlichem Erkenntnisstand ist bei dauerhafter Einhaltung des allgemeinen Staubgrenzwertes nicht mit einer Gesundheitsgefährdung im Sinne einer chronischen Bronchitis zu rechnen. Der vorgesehene allgemeine Staubgrenzwert gilt nur, wenn sichergestellt ist, dass eine genotoxische, fibrogene, allergisierende oder sonstige toxische Wirkung des Staubes nicht zu erwarten ist.

\section{Appendix B}

Teilnehmerliste der Konsensuskonferenz zur COPD-Leitlinie am 30.10.2005 in Kassel

Herr Dr. Helmut Berck

Patientenliga Atemwegserkrankungen e.V.

Lindenstr. 26

55130 Mainz

Patientenorganisationen

Frau Dr. Brigitte Bartuschka

Schillbachstr. 13

07743 Jena

Gast

Frau Dr. Uta Butt

Bergstr. 13

57223 Kreuztal-Ferndorf

Für das Protokoll

Herr Prof. Dr. Carl-Peter Criée

Ev. Krankenhaus Göttingen-Weende

Med. Klinik, Abtlg. Beatmungsmedizin/Schlaflabor

Pappelweg 5

37120 Bovenden/Lenglern

Deutsche Atemwegsliga e.V.

Herr Hans Dirmeier

Dtsch. Selbsthilfegruppe für Sauerstoff-Langzeit-Therapie(LOT) e.V.

Brunhuberstr. 23

83512 Wasserburg

Patientenorganisationen

Herr Prof. Dr. Adrian Gillissen

Städt. Kliniken St. Georg, Robert Koch-Klinik
Nikolai-Rumjanzew-Str. 100

04207 Leipzig

DGP

Herr Dr. Peter Kardos

Gemeinschaftspraxis \& Zentrum für Pneumologie, Allergologie, Schlafmedizin

Klinik Maingau Frankfurt am Main

Scheffelstr. 33

60318 Frankfurt

Deutsche Atemwegsliga, niedergelassene Pneumologen

Herr Prof. Dr. Dieter Köhler

Fachkrankenhaus, Kloster Grafschaft

Annostr. 1

57392 Schmallenberg

DGP

Herr Dr. Michael Köhler

Abt. Med. Wissenschaft Sektionsleiter Pneumologie, Boehringer Ingelheim Pharma GmbH \& Co. KG

Bingerstr.

55126 Ingelheim

Arzneimittelhersteller

Herr Prof. Dr. Helgo Magnussen

Krankenhaus Großhansdorf

Wöhrendamm 80

22927 Großhansdorf

DGP

Frau Dorothea Pfeiffer-Kascha

Zentralverband der Physiotherapeuten/Krankengymnasten

e.V.

Deutzer Freiheit $72-74$

50679 Köln

Physiotherapeuten

Herr Prof. Dr. Klaus F. Rabe

Department of Pulmonology, University of Leiden

Albinusdreef 9

2333 XA Leiden/Niederlande

GOLD

Herr Dr. Konrad Schultz

Fachklinik Allgäu

Peter-Heel-Str. 29

87459 Pfronten-Ried

Sektion Prävention und Rehabilitation der DGP

Herr Dr. Helmut Sitter

Inst. f. theor. Chirurgie, Zentrum für op. Medizin

Klinikum der Phillips-Universität Marburg

Baldinger Str. 1

35033 Marburg

Moderation

Herr Hans-Jürgen Smith

VIASYS Healthcare GmbH

Leibnizstr. 7

97204 Höchberg

Gerätehersteller

Herr Dr. Diethard Sturm

Weinkellerstr. 20

09337 Hohenstein-Ernstthal

Deutscher Hausärzteverband

Herr Prof. Dr. Helmut Teschler

Ruhrlandklinik

Tüschener Weg 40

45239 Essen

Klinische Pneumologie 
Herr Prof. Dr. Claus Vogelmeier

Universitätsklinikum, Schwerpunkt Pneumologie

Baldinger Str.

35033 Marburg

Deutsche Atemwegsliga

Herr Karsten Wagner-Gillen

GlaxoSmith-Kline GmbH

Theresienhöhe 11

80339 München

Arzneimittelhersteller

Herr Prof. Dr. Tobias Welte

Med. Hochschule Hannover, Pneumologie

Carl-Neuberg-Str. 1

30625 Hannover

Deutsche Atemwegsliga

Herr Prof. Dr. Ralf Wettengel

Schillbachstr. 13

07743 Jena

Deutsche Atemwegsliga

Herr Prof. Dr. Heinrich Worth

Med.Klinik I/Klinikum Fürth

Jakob-Henle-Str. 1

90766 Fürth

Deutsche Atemwegsliga

\section{Literatur}

1 Worth $H$, Buhl R, Cegla $U$ et al. Leitlinie der Deutschen Atemwegsliga und der Deutschen Gesellschaft für Pneumologie zur Diagnostik und Therapie von Patienten mit chronisch obstruktiver Bronchitis und Lungenemphysem (COPD). Pneumologie 2002; 56: 704 - 738

2 Global Initiative for Chronic Obstructive Lung Disease (GOLD). Global strategy for the diagnosis, management, and prevention of chronic obstructive pulmonary disease. Updated 2005 http://www.goldcopd.com(goldwr2005.pdf,

3 Celli BR, MacNee W and committee members. Standards for the diagnosis and treatment of patients with COPD: a summary of the ATS/ ERS position paper. Eur Respir J 2004; 23: 932 - 946

4 National Collaborating Centre for Chronic Conditions, National Institute of Clinical Excellence (NICE). Chronic obstructive pulmonary disease. National clinical guideline on management of chronic obstructive pulmonary disease in adults in primary and secondary care. Thorax 2004; 59 Suppl 1: 1-232

5 Pauwels RA, Buist AS, Calverley PMA et al. Global strategy for the diagnosis, management, and prevention of chronic obstructive pulmonary disease. Am J Respir Crit Care Med 2001; 163: 1256-1276

6 ATS Statement. Standards for the diagnosis and care of patients with chronic obstructive pulmonary disease. Am J Respir Crit Care Med 1995; 152 : S77-S120

7 Siafakas NM, Vermeire P, Pride NB et al. ERS-Consensus statement. Optimal assessment and management of chronic obstructive pulmonary disease. Eur Respir J 1995; 8: 1388 - 1420

8 BTS. Guidelines for the management of chronic obstructive pulmonary disease. Thorax 1997; 52 (Suppl 5): S1 -S28

9 Canadian Respiratory Review Panel. Guidelines for the treatment of chronic obstructive pulmonary disease (COPD). Monograph, 1998

10 Laitinen L, Koskela K et al. Chronic bronchitis and chronic obstructive pulmonary disease: Finnish national guidelines for prevention and treatment 1998 - 2007. Respir Med 1999; 93: 297-332

11 Lorenz W, Ollenschläger $G$ et al. Das Leitlinienmanual von AWMF und ÄZQ. ZaeFQ 2001; 95 (Suppl. I): 41 - 43

12 Arbeitsgemeinschaft der Wissenschaftlichen Medizinischen Fachgesellschaften. Erarbeitung von Leitlinien für Diagnostik und Therapie. Methodische Empfehlungen. Abrufbar unter: http://www.uni-duesseldorf.de/WWW/AWMF,

13 Society for Medical Decision Making. Committee on standardization of clinical algoritms. Proposals for clinical algorithm standards. Med Decis Making 1992; 12: 149-154
14 Report of an expert committee: Definition and diagnosis of pulmonary disease with special reference to chronic bronchitis and emphysema. WHO Techn Rep Ser 1961; 213: 14 - 19

15 Ciba guest symposium report: Terminology, definitions and classifications of chronic pulmonary emphysema and related conditions. Thorax 1959; 14: 286-299

16 Konietzko N, Fabel H. Weißbuch Lunge 2000. Stuttgart-New York: Thieme,

17 Statistisches Bundesamt. Statistisches Jahrbuch, Jahrgänge 1968 - 1999. Stuttgart-Mainz: Kohlhammer,

18 Murray CJL, Lopez AD. The global burden of disease: A comprehensive assessment of mortality and disability from diseases. Injuries and risk factors in 1990 and projected to 2020. Cambridge: Harvard University Press, 1996

19 Lopez AD, Murray CJL. The global burden of disease, 1990-2020. Nature Med 1998; 4: 1241 - 1243

20 Nowak D, Berger K, Lioppert B et al. Epidemiology and health economics of COPD across Europe. A Critical Analysis. Treat Respir Med 2006; 4: 381 - 395

21 Fabel H, Konietzko N (Hrsg). Weißbuch Lunge. Stuttgart, New York: Thieme Verlag, 2005

22 Rulff $L K$, Volmer T, Nowak $D$. The economic inpact of smoking in Germany. Eur Respir J 2000; 16: 385 - 390

23 Rychlik R, Pfeil T, Daniel D et al. Zur sozioökonomischen Relevanz akuter Exacerbationen der chronischen Bronchitis in der Bundesrepublik Deutschland. Dtsch Med Wschr 2001; 126: 353-359

24 Nowak D, Dietrich ES, Oberender P et al. Krankheitskosten von COPD in Deutschland. Pneumologie 2004; 58 (12): 837 - 844

25 Laurell CB, Eriksson S. The electrophoretic alpha- 1 globulin pattern of serum in alpha- 1 antitrypsin deficiency. Scand J Clin Lab Invest 1963; $15: 132-140$

26 Orie NGM, Sluiter HJ, De Vreis $K$ et al. Bronchitis, an international symposium. Assen, Netherlands: Royal Vangorum, 1961: 43-59

27 Svanes C, Omenaas E, Heuch JM et al. Birth characteristics and asthma symptoms in young adults: results from a population-based cohort study in Norway. Eur Respir J 1998; 12: 1366-1370

28 Todisco T, de Benedictis FM, Iannacci L et al. Mild prematurity and respiratory functions. Eur J Pediatr 1993; 152: 55-58

29 Stein CE, Kumaran K, Fall CH et al. Relation of fetal growth to adult lung function in South India. Thorax 1997; 52: 895-899

30 Morgan WJ. Maternal smoking and infant lung function: further evidence for an in utero effect. Am J Respir Crit Care Med 1998; 158: $689-690$

31 U.S. Surgeon General. The health consequences of smoking: chronic obstructive pulmonary disease. Washington DC: U.S. Department of health and human services, 1984. Publication No. 84-50205

32 Leuenberger P, Schwartz J, Ackermann-Liebrich $U$ et al. Passive smoking exposure in adults and chronic respiratory symptoms (SAPALDIA Study). Swiss Study on air pollution and lung diseases in adults. SAPALDIA Team. Am J Respir Crit Care Med 1994; 150: 1222-1228

33 Dayal HH, Khuder S, Sharrar R et al. Passive smoking in obstructive respiratory disease in an industrialized urban population. Environ Res 1994; 65: 161 - 171

34 Holt PG. Immune and inflammatory function in ciacutegarette smokers. Thorax 1987; 42: 241 - 249

35 Kauffmann F, Droulet D, Lellouch J et al. Twelve years spirometric changes among Paris area workers. Int J Epidemiol 1979; 8: 201 -212

36 Niewoehner DE. Anatomic and pathophysiological correlations in COPD. In: Baum Gl, Crapo JD, Celli BR, Karlinsky JB (eds). Textbook of pulmonary diseases. Philadelphia: Lippincott-Raven, 1998: 823 842

37 Chen JC, Mannino MD. Worldwide epidemiology of chronic obstructive pulmonary disease. Current Opin Pulmon Med 1995; 5: 93-99

38 Behera D, Jindal SK. Respiratory symptoms in Indian women using domestic cooking fuels. Chest 1991; 100: $385-388$

39 Georgopoulos D, Anthonisen NR. Symptoms and signs of COPD. In: Cherniack NS (ed). Chronic obstructive pulmonary disease Toronto: Saunders, 1991: 357-363

40 Criée; C-P, Berdel D, Heise D et al. Empfehlungen der Deutschen Atemwegsliga zur Spirometrie. Dustri-Verlag Dr. Karl Feistle, ISBN 3-87185-364-X),

41 Pellegrino R, Viegi G, Brusasco Vet al. Interpretative strategies for lung function tests. Eur Respir J 2005; 26: 948 - 968

42 Taube MD, Lehnigk B, Paasch $K$ et al. Factor analysis of changes in dyspnea and lung function parameters after bronchodilatation in 
chronic obstructive pulmonary disease. Am J Respir Crit Care Med 2000; $162: 216-220$

43 O'Donnell DE, Lam M, Webb KA. Measurement of symptoms, lung hyperinflation, and endurance during exercise in chronic obstructive pulmonary disease. Am J Respir Crit Care Med 1998; 158: 1557 1565

44 O'Donnell DE, Lam M, Webb KA. Spirometric correlates for improvement in exercise performance after anticholinergic therapy in chronic obstructive pulmonary disease. Am J Respir Crit Care Med 1999; 160: $542-549$

45 Marin JM, Carrizo SJ, Gascon $M$ et al. Inspiratory capacity, dynamic hyperinflation, breathlessness, and exercise performance during the 6-minute-walk test in chronic obstructive pulmonary disease. Am J Respir Crit Care Med 2001; 163: 1395-1399

46 Traver GA, Cline MG, Burrows B. Predictors of mortality in COPD. Am Rev Respir Dis 1979; 119: 895-902

47 Burrows $B$. The course and prognosis of different types of chronic airflow limitation in a general population sample from Arizona: comparison with the Chicago "COPD" series. Am Rev Respir Dis 1989; 140: S92 - S94

48 Seemungal TA, Donaldson GC, Bhownik A et al. Time course and recovery of exacerbations in patients with chronic obstructive pulmonary disease. Am J Respir Crit Care Med 2000; 161: 1608 - 1613

49 Calverley PMA, Burge PS, Spencer S et al. Bronchodilator reversibility testing in chronic obstructive pulmonary disease. Thorax 2003; 58 : 659-664

50 Berger $R$, Smith $D$. Effect of an inhaled metaproterenol on exercise performance in patients with stable "fixed" airway obstruction. Am Rev Respir Dis 1988; 138: 626-629

51 Casaburi R, Mahler DA, Jones PW et al. A long-term evaluation of once-daily inhaled tiotropium in chronic obstructive pulmonary disease. Eur Respir J 2002; 19: 217 - 224

52 Vincken W, Noord JA von, Greefhorst AP et al. Improved health outcomes in patients with COPD during 1 yr's treatment with tiotropium. Eur Respir J 2002; 19: 209-216

53 Celli B, ZuWallack R, Wang $S$ et al. Improvement in Resting Inspiratory Capacity and Hyperinflation With Tiotropium in COPD Patients With Increased Static Lung Volumes. Chest 2003; 124: 1743-1748

54 Calverley P, Pauwels R, Vestbo J et al. Combined salmeterol and fluticasone in the treatment of chronic obstructive pulmonary disease: a randomised controlled trial. Lancet 2003; 361: 449-456

55 Callahan CM, Cirrus RS, Katz BP. Oral corticosteroid therapy for patients with stable chronic obstructive pulmonary disease: a metaanalysis. Ann Intern Med 1991; 114: 216-223

56 Kardos $P$, Brutsche $M$, Buhl $R$ et al. Kombination von Asthma und COPD - häufiger als man denkt? Pneumologie 2006; 60: 366 - 372

57 Casanova C, Cote C, de Torres JP et al. Inspiratory-to-Total Lung Capacity Ratio Predicts Mortality in Patients with Chronic Obstructive Pulmonary Disease. Am J Respir Crit Care Med 2005; 171: 591 - 597

58 Roberts CM, Bugler JR, Melchor $R$ et al. Value of pulse oximetry for long-term oxygen therapy requirement. Eur Respir J 1993; 6: 559562

59 Hughes JMB, Pride NB. In defense of the carbon monoxide transfer coefficent Kco (TL/VA). Eur Respir J 2001; 17: 168 - 174

60 McLean A, Warren PM, Gilooly M et al. Microscopic and macroscopic measurement of emphysema: relation to carbon monoxide transfer. Thorax 1992; 47: 14-19

61 Gevenois PA, de Vuyst P, de Maertelaer V et al. Comparison of computed density and microscopic morphometry in pulmonary emphysema. Am J Respir Crit Care Med 1996; 154: 187-192

62 Gould GA, Redpath AT, Ryan M et al. Lung CT density correlates with measurements of airflow limitation and the diffusing capacity. Eur Respir J 1991; 4: 141 - 146

63 Worth H, Breuer HWM, Dorow P et al. Deutsche Gesellschaft für Pneumologie. Empfehlungen zur Durchführung und Bewertung von Belastungsuntersuchungen in der Pneumologie. Pneumologie 1998; 52: $225-231$

64 Thurlbeck WM, Simon G. Radiographic appearance of the chest in emphysemas. Am J Roentgenol 1978; 130: 429-440

65 Morgan MDL, Denison DM, Stricklund B. Value of computed tomography for selecting patients with bullous emphysema. Thorax 1986; 41: $855-862$

66 Lehtonen J, Sutinen S, Ikaheimo M et al. Electrocardiographic criteria for the diagnosis of right ventricular hypertrophy verified at autopsy. Chest 1988; 93: 839-842
67 Niedermeier J, Barner A, Borst M et al. Deutsche Gesellschaft für Pneumologie. Empfehlungen zur Anwendung der Echokardiographie in der pneumologischen Diagnostik. Pneumologie 1998; 52: 519-521

68 O'Donnell DE, Forkert L, Webb KA. Evaluation of bronchodilator responses in patients with "irreversible" emphysema. Eur Respir J 2001; 18: $914-920$

69 Begin P, Grassino A. Inspiratory muscle dysfunction and chronic hypercapnia in chronic obstructive pulmonary disease. Am Rev Respir Dis 1991; 143: 901 - 903

70 Baldi S, Miniati M, Bellina CR et al. Relationship between extent of pulmonary emphysema by high-resolution computed tomography and lung elastic recoil in patients with chronic obstructive pulmonary disease. Am J Respir Crit Care Med 2001; 169: 585 - 589

71 Wolkove N, Dacezman E, Colacone A et al. The relationship between pulmonary function and dyspnoea in obstructive lung disease. Chest 1989; 96: $1247-1251$

72 Connolly MC, Crowley JJ, Charan NB et al. Reduced subjective awareness of bronchoconstriction by metacholine in elderly asthmatic and normal subjects as measured on a simple awareness scale. Thorax 1992; 47: 410-413

73 Celli BR, Cote CG, Marin JM et al. The Body-Mass Index, Airflow Obstruction, Dyspnea, and Exercise Capacity Index in Chronic Obstructive Pulmonary Disease. NEJM 2004; 350: 1005 - 1012

74 Stavem K, Sandvik L, Erikssen J. Can Global Initiative for Chronic Obstructive Lung Disease Stage 0 provide prognostic information on long-term mortality in men? Chest 2006; 130: 318-325

75 Vestbo J, Lange P. Can GOLD Stage 0 Provide Information of Prognostic Value in Chronic Obstructive Pulmonary Disease? Am J Respir Crit Care Med 2002; 166: 329-332

76 Vestbo J, Lange P. Can GOLD Stage 0 Provide Information of Prognostic Value in Chronic Obstructive Pulmonary Disease? Am J Respir Crit Care Med 2002; 166: 329-332

77 Weiner P, Weiner M, Azgad Y et al. Inhaled budesonide therapy for patients with stable COPD. Chest 1995; 108: 1568-1571

78 Kardos P, Brutsche M, Buhl R et al. Kombination von Asthma und COPD - häufiger als man denkt? Pneumologie 2006; 60 (6): 366 372

79 Seemugal TAR, Donaldson GC, Bhowmik A et al. Time Course and Recovery of Exacerbations in Patients with Chronic Obstructive Pulmonary Disease. Am J Respir Crit Care Med 2000; 161: 1608 - 1613

80 Schols AMWJ, Slangen J, Volorris A et al. Wouters. Weigth loss is a reversible factor in the prognosis of COPD. Am J Respir Crit Care Med 1998; $157: 1791$ - 1797

81 Hurst R, Donaldson GC, Wilkinson TMA et al. Epidemiological relationships between the common cold and exacerbation frequency in COPD. Eur Respir J 2005; 26: 846-852

82 Donaldson GC, Seemungal TAR, Bhowmik A et al. Relationship between exacerbation frequency and lung function decline in chronic obstructive pulmonary disease. Thorax 2002; 57: 847-852

83 Anthonisen NR, Skeans MA, Wise RA et al. The effects of a smoking cessation intervention on 14.5-year mortality: a randomized clinical trial. Ann Intern Med 2005; 142 (4): 233 - 239

84 Thomas R. School-based programmes for preventing smoking. Cochrane Database Syst Rev 2002; (4): CD001293

85 Fichtenberg CM, Glantz SA. Effect of smoke-free workplaces on smoking behaviour: systematic review. BMJ 2002; 325 (7357): 188

86 Lovato C, Linn F, Stead LF et al. Impact of tobacco advertising and promotion on increasing adolescent smoking behaviours. Cochrane Database Syst Rev 2003; (4): CD003439

87 Wilson DH, Wakefield MA, Steven ID et al. "Sick of smoking" evaluation of a targeted minimal smoking cessation intervention in general practice. Med J Aust 1990; 152: 518 - 521

88 Britton J, Knox A. Helping people to stop smoking: the new smoking cessation guidelines. Thorax 1999; 54: 1 - 2

89 Kottke TE, Ballista RN, de Friese GH et al. Attributes of successful smoking cessation interventions in medical practise. A meta-analysis of 39 controlled trials. JAMA 1988; 259: 2883-2889

90 Ockene JK, Kristeller J, Goldberg R et al. Increasing the efficacy of physician deliverd smoking interventions: a randomized controlled clinical trial. J Gen Intern Med 1991; 6: 1 - 9

91 Fiore MC, Bailey WC, Cohen SJ. Smoking cessation: information for specialists. U.S. department of health and human services. Public health service. Agency for health care policy and research and centers for disease control and prevention. Rockville. M.D. AHCPR Publication No. 96-0694, 1996 
92 The tobacco use and dependence clinical practice guideline panel, staff, and consortium repesentatives. A clinical practice guideline for treating tobacco use and dependence. JAMA 2000; 83: 32443254

93 American Medical Association. Guidelines for the diagnosis and treatment of nicotine dependence. How to help patients stop smoking. Washington D.C.: American Medical Association, 1994

94 Glynn TJ, Manley MW. How to help your patients stop smoking. A national cancer institute manual for physicians. U.S. Department of health and human service, public health service. National institutes of health. National Cancer Institute, Bethesda. M.D. NIH Publication 90-3064, 1990

95 Anthonisen NR, Connett JE, Kiley JP et al. Effects of smoking intervention and the use of an inhaled anticholinergic bronchodilator on the rate of decline of $\mathrm{FEV}_{1}$ : The Lung Health Study. JAMA 1994; 272: $1497-1505$

96 Arzneimittelkommission der Deutschen Ärzteschaft. Empfehlungen zur Therapie der Tabakabhängigkeit. Köln: AVP-Reihe, 2001

97 Jorenby RE, Leischow SJ, Nides MA et al. A controlled trial of sustained release bupropion, a nicotine patch, or both for smoking cessation. $\mathrm{N}$ Engl J Med 1999; 340: 685-691

98 Tashkin DP, Kanner RJ, Buist S et al. Smoking cessation in patients with chronic obstructive pulmonary disease: a double blind, placebo-controlled randomised trial. Lancet 2001; 357: 1571-1575

99 WHO. Tobacco free initiative: Policies for public Health: www.who// int/toh/world notto-bacco 99

100 Fiore MC, Bailey WC, Cohen SJ et al. Treating tobacco use as dispendence : A clinical practical guideline. Rockville, M.D. US Dept Health and Human Services. A. public health service report, 2000

101 Schwartz JL. Review and evalution of smoking cessation methods: United States and Canada, 1978 - 1985. Bethesda, M.D.: National Institute of Health. NIH publication 87-2940, 1987

102 Fiore MC, Smith SS, Jorenby DE et al. The effectiveness of the nicotine patch for smoking cessation. A meta-analysis. JAMA 1994; 271: $1940-1947$

103 Sachs DE, Benowitz NL. Individual medical treatment for tobacco dependence [editorial; comment]. Eur Respir J 1996; 9: 629-631

104 Murray RP, Connett JE, Rand CS et al. Anthonisen. Persistence of the effect of the Lung Health Study (LHS) smoking intervention over eleven years. Prev Med 2002; 35 (4): 314-319

105 Bundesversicherungsanstalt für Angestellte (BfA). Rehabilitationsbedürftigkeit und Indikationsübergreifende Problembereiche - Leitlinien für den Beratungsärztlichen Dienst der BfA 2002: http://www.bfa.de/nn 5910/de/Inhalt/Publikationen/Sonstige/Infos $20 f$ C3 BCr 20 C3 84rzte Dokumente/Reha Bed C3 Bcrftigkeit 20 2F 20 Indiaktions C3 Bcbergreifend.html,

106 Eisner MD, Balmes J, Yelin EH et al. Directly measured secondhand smoke exposure and COPD health outcomes. BMC Pulm Med 2006Jun 6; 6: 12

107 Berufsgenossenschaftlicher Grundsatz für arbeitsmedizinische Vorsorgeuntersuchungen „G 23 - Obstruktive Atemwegserkrankungen“. Hrsg.: Hauptverband der gewerblichen Berufsgenossenschaften, St. Augustin,

108 Nichol K, Margolis L, Wourenma J et al. The efficacy and cost effectiveness of vaccination against influenza among elderley persons living in the community. N Engl J Med 1994; 331: 778 - 784

109 Hak E, Essen GA van, Buskens $E$ et al. Is immunising all patients with chronic lung disease in the community against influenza cost effective? Evidence from a general based clinical prospective cohort study in Utrecht. The Netherlands. J Epidemiol Community Health 1998; 52: $120-125$

110 Peters PH. Long-term use of oseltamivir for the prophylaxis of influenza in a vaccinated frail older population. J Am Geriatr Soc 2001; 49 (8): $1025-1031$

111 Hayden FG, Belshe R, Villanueva C et al. Management of influenza in households: a prospective, randomized comparison of oseltamivir treatment with or without postexposure prophylaxis. J Infect Dis 2004; 189 (3): $440-449$

112 Simberkoff MS, Cross AP, Al-Ibrahim M et al. Efficacy of pneumococcal vaccine in high-risk patients. Results of a Veterans Administration Cooperative Study. N Engl J Med 1986; 315: 1318 - 1327

113 Williams JHJ, Moser KM. Pneumococcal vaccine and patients with chronic lung disease. Ann Intern Med 1986; 104: 106 - 109

114 Christenson B, Lundbergh P, Hedlund J et al. Effects of a large-scale intervention with influenza and 23 -valent pneumococcal vaccines in adults aged 65 years or older: a prospective study. Lancet 2001; 357: $1008-1011$

115 Davis AL, Aranda P, Schiffman G et al. Pneumococcal infection and immunologic response to pneumococcal vaccine in chronic obstructive pulmonary disease. A pilot study. Chest 1994; 92: 204-212

116 Schaberg T. Pneumokokkenschutzimpfung. Pneumologie 2001; 55: $543-545$

117 Ständige Impfkommission am Robert Koch-Institut (STIKO). Epidemiologisches Bulletin des RKI 2001; 28: 203 -218

118 Pauwels RA, Lofdahl CG, Laitinen LA et al. Long-term treatment with inhaled budesonide in persons with mild chronic obstructive pulmonary disease who continue smoking. N Engl J Med 1999; 340: 1948 1953

119 Vestbo J, Sorensen T, Langer P et al. Longterm effect of inhaled budesonide in mild and moderate chronic obstructive pulmonary disease: a randomised controlled trial. Lancet 1999; 355: 1819-1823

120 Burge PS, Calverley MA, Jones PW et al. Randomised, placebo-controlled study of fluticasone proprionate in patients with moderate to severe chronic obstructive pulmonary disease; the Isolde trial. Br Med J 2000; 320: $1297-1303$

121 The Lung Health Study Research Group. Effect of inhaled triamcinolone on the decline in pulmonary function in chronic obstructive pulmonary disease. N Engl J Med 2000; 343: 1902 - 1909

122 Schayck CP van, Dompeling E, Heerwaarden CLA van et al. Bronchodilator treatment in modern asthma or chronic bronchitis. Continuous or on demand. A randomised controlled study. BMJ 1991; 303: $1426-1431$

123 Voshaar T, App EM, Berdel D et al. Empfehlungen für die Auswahl von Inhalationssystemen für die Medikamentenverabreichung. Pneumologie 2001; 55: 579-586

124 Dahl R, Greefhorst LA, Nowak $D$ et al. Inhaled formoterol dry powder versus ipratropium bromide in chronic obstructive pulmonary disease. Am J Respir Crit Care Med 2001; 164: 778 - 784

125 Mahler DA, Donohue JF, Barbee RA et al. Efficacy of salmeterol xinafoate in the treatment of COPD. Chest 1999; 115: 957-965

126 Karpel JP, Pesin J, Greenberg D et al. A comparison of the effects of ipratropium bromide and metaproterenol sulfate in acute exazerbations of COPD. Chest 1990; 98: 835-839

127 Patrick DM, Dales RE, Stark RM et al. Severe exacerbations of COPD and asthma. Incremental benefit of adding ipratropium to usual therapy. Chest 1990; 98: 295-297

128 Braun SR, Mc Kenzie WN, Copeland C et al. A comparison of the effect of ipratropium and albuterol in the treatment of chronic airways disease. Arch Intern Med 1989; 149: 544-547

129 Tashkin DP, Ashutosh K, Bleeker ER et al. Comparison of the anticholinergic bronchodilator ipratropiumbromide with metaproterenol in chronic obstructive pulmonary disease: a multicenter study. Am J Med 1986; 81 (Suppl. 5a): 1411 - 1419

130 COMBIVENT Inhalation Aerosol Study Group. In chronic obstructive pulmonary disease, a combination of ipratropium and albuterol is more effective than either agent alone: an 85-day multicenter trial. Chest 1994; 105: $1411-1419$

131 Rennard SI. Extended bronchodilator treatment in chronich obstructive pulmonary disease. European Respiratory Review 1996; 6: $282-286$

132 Barnes PJ. The pharmacological properties of tiotropium. Chest 2000; 117: 63S-66S

133 Calverley PMA. The future of tiotropium. Chest 2000; 117: 67S - 69S

134 Noord JA van, Aumann JL, Janssens E et al. Effects of tiotropium with and without formoterol on airflow obstruction and resting hyperinflation in patients with COPD. Chest 2006 Mar; 129 (3): 509-517

135 O'Donnell DE, Fluge T, Hamilton A et al. Effects of tiotropium on lung hyperinflation, dyspnoea and exercise tolerance in COPD. Eur Respir J 2004; 23: 832-840

136 Niewoehner DE, Rice K, Cote $C$ et al. Prevention of exacerbations of chronic obstructive pulmonary disease with tiotropium, a once-daily inhaled anticholinergic bronchodilator: a randomized trial. Ann Intern Med 2005; 143: 317-326

137 Dusser D, Bravo ML, Iacono $P$. The effect of tiotropium on exacerbations and airflow in patients with COPD. Eur Respir J 2006; 27: $547-555$

138 Casaburi R, Kukafka D, Cooper CB et al. Improvement in exercise tolerance with the combination of tiotropium and pulmonary rehabilitation in patients with COPD. Chest 2005; 127: 809-817 
139 Brusasco V, Hodder R, Miravitlles $M$ et al. Health outcomes following treatment for six months with once daily tiotropium compared with twice daily salmeterol in patients with COPD. Thorax 2003 May; 58 (5): 399-404, Erratum in: Thorax 2005 Feb; 60 (2): 105

140 Campbell M, Eliraz A, Johannson $G$ et al. Formoterol for maintenance and as-needed treatment of chronic obstructive pulmonary disease. Respiratory medicine 2006; 99: 1511 - 1520

141 Nisar M, Earis JE, Pearson MG et al. Acute bronchodilator trials in chronic obstructive pulmonary disease. Am Rev Respir Dis 1992; 146: $555-559$

142 Ulrik CS. Efficacy of inhaled salmeterol in the management of smokers with chronic obstructive pulmonary disease: a single centre randomised, double blind, placebo controlled, crossover study. Thorax 1995; 50: 750-754

143 Grove A, Lipworth BJ, Reid P et al. Effects of regular salmeterol on lung function and exercise capacity in patients with chronic obstructive airways disease. Thorax 1996; 51: 689-693

144 Boyd G, Mirice AH, Pounsford JG et al. An evaluation of salmeterol in the treatment of chronic obstructive pulmonary disease (COPD). Eur Respir J 1997; 10: 815-821

145 Maesen BLP, Wedermann CIJ, Duarkens VAM et al. Effects of formoterol in apparently poorly reversible chronic obstructive pulmonary disease. Eur Respir J 1999; 13: 1103 - 1108

146 O'Donnell DE, Voduc N, Fitzpatrick $M$ et al. Effect of salmeterol on the ventilatory response to exercise in chronic obstructive pulmonary disease. Eur Respir J 2004; 24: 86-94

147 Jones PW, Bosh TK. Quality of life changes in COPD patients treated with salmeterol. Am J Respir Crit Care Med 1997; 155: 1283-1289

148 Peach H, Pathy MS. Follow-up study of disability among elderly patients discharged from hospital with exacerbations of chronic bronchitis. Thorax 1981; 36: 585-589

149 Cazzola M, Imperatore F, Salzillo A et al. Cardiac effects of formoterol and salmeterol in patients suffering from COPD with preexisting cardiac arrhythmia. Chest 1998; 114: $411-415$

150 Murciano D, Auclair M-A, Pariente $R$ et al. A randomized controlled trial of theophylline in patients with severe chronic obstructive pulmonary disease. N Engl J Med 1989; 320: 1512 - 1525

151 McKay SE, Howie CA, Thomson AH et al. Value of theophylline treatment in patients handicapped by chronic obstructive pulmonary disease. Thorax 1993; 48: 227-232

152 Mulloy E, McNicholas WT. Theophylline improves gas exchange during rest, exercise and sleep in severe chronic obstructive pulmonary disease. Am Rev Respir Dis 1993; 148: 1030-1036

153 Evans WW. Plasma theophylline concentrations, six minute walking distances and breathlessness in patients with chronic airflow obstruction. BMJ 1984; 289: 1649-1651

154 Cooper CB, Davidson AC, Cameron IR. Aminophylline, respiratory muscle strength and exercise tolerance in chronic obstructive airway disease. Clin Respir Physiol 1987; 23: 15-22

155 Murciano D, Aubier M, Lecolguic Y et al. The effects of theophylline on diaphragmatic strength an exercise tolerance in patients with chronic obstructive pulmonary disease. N Engl J Med 1984; 322: 349353

156 Williamson BH, Milligan C, Griffiths K et al. Thompson. An assessment of major and minor side effects of theophylline. Aust NZ J Med 1988; 19: 539

157 Kirsten $D$, Wegner RE, Jörres RA et al. Effects of theophylline withdrawal in severe chronic obstructive pulmonary disease. Chest 1993; 104: $1101-1107$

158 The COMBIVENT Inhalation Solution Study Group. Routine nebulized ipratropium and albuterol together are better than either alone in COPD. Chest 1997; 112: $1514-1521$

159 Taylor DR, Buick B, Kinney C et al. The efficacy of orally administered theophylline, inhaled salbutamol and a combination of the two as chronic therapy in the management of chronic bronchitis with reversible airflow obstruction. Am J Respir Dis 1985; 131: 747-751

160 Friedman M, Serby C, Menjoge S et al. Pharmaeconomic evaluation of a combination of ipratropium plus albuterol compared with ipratropium alone and albuterol alopne in COPD. Chest 1999; 115: 635-641

161 D'Urzo AD, de Salvo MC, Ramirez-Rivera A et al. In patients with COPD, treatment with a combination of formoterol and ipratropium is more effective than a combination of salbutamol and ipratropium. Chest 2001; 119: $1347-1356$
162 Guyatt GH, Townsend M, Pugsley SO et al. Bronchodilators in chronic air-flow limitation: effects on airway function, exercise capacity and quality of life. Am Rev Respir Dis 1987; 135: 1069- 1074

163 Noord JA van, Aumann J-L, Janssens E et al. Comparison of tiotropium once daily, formoterol twice daily and both combined once daily in patients with COPD. Eur Respir J 2005; 26: 214-222

164 O'Driscoll BR, Kay EA, Taylor RJ et al. A long-term prospective assessment of home nebulizer treatment. Respir Med 1992; S6: 317- 325

165 Gan WQ Man SFP, Sin DD. Effects of inhaled corticosteroids on sputum cell counts in stable chronic obstructive pulmonary disease: a systematic review and a meta-analysis. BMC Pulmonary Medicine 2005; $5: 3$

166 Senderovitz T, Vestbo J, Frandsen J et al. Steroid reversibility test followed by inhaled budesonide or placebo in outpatients with stable chronic obstructive pulmonary disease. The Danish Society or Respiratory Medicine. Respir Med 1999; 93: 715-718

167 Rice KL, Rubins JB, Lebhahn F et al. Withdrawal of chronic systemic corticosteroids in patients with COPD: a randomized trial. Am J Respir Crit Care Med 2000; 162: 174- 178

168 Calverley PM, Boonsawat W, Cseke Z et al. Maintenance therapy with budesonide and formoterol in chronic obstructive pulmonary disease. Eur Respir J 2003; 22: 912 - 919

169 Szafranski W, Cukier A, Ramirez A et al. Efficacy and safety of budesonide/formoterol in the management of chronic obstructive pulmonary disease. Eur Respir J 2003; 21: $74-81$

170 Wouters EF, Postma DS, Fokkens B et al. Withdrawal of fluticasone propionate from combined salmeterol/fluticasone treatment in patients with COPD causes immediate and sustained disease deterioration: a randomized controlled trial. Thorax 2005; 60: 480-487

171 Valk P van der, Monninkhof E, Palen J van der et al. Effect of discontinuation of inhaled corticosteroids in patients with chronic obstructive pulmonary disease: the COPE study. Am J Respir Crit Care Med 2002; 166: 1358-1363

172 Kardos P, Wencker M, Glaab T et al. Impact of salmeterol/fluticasone versus salmeterol on exacerbations in severe chronic obstructive pulmonary disease. Am J Respir Crit Care Med 2007; 175: 144-149

173 Decramer M, Lacquet LM, Fagard R et al. Corticosteroids contribute to muscle weakness in chronic airflow obstruction. Am J Respir Crit Care Med 1994; 150: $11-16$

174 Decramer M, Stas KJ. Corticosteroid-induced myopathy involving respiratory muscles in patients with chronic obstructive pulmonary disease or asthma. Am J Respir Dis 1992; 146: 800-802

175 Schols AMWJ, Wesseling G, Keser ADM et al. Dose dependent increased mortality risk in COPD patients treated with oral glucocorticoids. Eur Respir J 2001; 17: 337 - 342

176 Mahler DA, Wire P, Horstmann D et al. Effectiveness of fluticasone propionate and salmeterol combination delivered via the diskus device in the treatment of chronic obstructive pulmonary disease. Am J Respir Crit Care Med 2002; 166: 1084- 1091

177 Hanania NA, Darken P, Horstman D et al. The efficacy and safety of fluticasone propionate (250 micro g)/salmeterol (50 micro g) combined in the diskus inhaler for the treatment of COPD. Chest 2003; 124 : $834-843$

178 Dal Negro R, Pomari C, Tognella $S$ et al. Salmeterol \& fluticasone 50 micro $\mathrm{g} / 250$ microg bid in combination provides a better long-term control than salmeterol 50 micro g bid alone and placebo in COPD patients already treated with theophylinne. Pulmonary Pharmacology and Therapeutics 2003; 16: $241-246$

179 Maltais F, Hamilton A, Marciniuk D et al. Improvement in symptomlimited exercise performance over $8 \mathrm{~h}$ in pateints with COPD. Chest 2005; 128: $1168-1178$

180 Weiner P, Magadle R, Berar-Yanay $N$ et al. The cumulatie effect of long-acting bronchodilators, exercise,and inspiratory muscle training in the perception of dyspnea in patients with advanced COPD. Chest 2000; 118: $672-678$

181 Liesker JJW, De Velde V van, Meysman $M$ et al. Effects of formoterol (Oxis Turbuhaler) and ipratropium on exercise capacity in patients with COPD. Respir Med 2002; 96: 559-566

182 Boyd G, Morice AH, Pounsford JC et al. An evaluation of salmeterol in the treatment of chronic obstructive pulmonary disease (COPD). Eur Respir J 1997; $10: 815-821$

183 Rennard StI, Anderson W, ZuWallack $R$ et al. Use of long-acting inhaled beta $_{2}$-adrenergic agonist Salmeterol xinafoate, in patients wirh chronic obstructive pulmonary disease. Am J Respir Crit Care Med 2001; 163: 1087-1092 
184 Man WDC, Mustfa N, Nikoletou D et al. Effecr of Salmeterol on respiratory muscle activity during exercise in poorly reversible COPD. Thorax 2004; 59: 471-476

185 Aalbers R, Ayres J, Backer V et al. Formoterol in patients with chronic obstructive pulmonary disease: a randomized, controlled, 3-month trial. Eur Respir J 2002; 19: 936 - 943

186 Wadbo M, Löfdahl C-G, Larsson K et al. Effects of formoterol and ipratropiumbromide in COPD: a 3-month placebo-controlled study. Eur Respir J 2002; 20: 1138 - 1146

187 Grassi C and the multicenter Study Group. Longterm oral acetylcysteine in chronic bronchitis. A double blind controlled study. Eur J Respir Dis 1980; 61 (Suppl. 111): $93-108$

188 Boman G, Bäcker U, Larsson $S$ et al. Oral acetylcysteine reduces exacerbation rate in chronic bronchitis. Report of a trial organized by the Swedish Society for Pulmonary Diseases. Eur J Respir Dis 1983; 64: $405-415$

189 Jackson M, Barnes J, Cooksey P. Efficacy and tolerability of oral acetylcysteine (Fabrol) in chronic bronchitis: A double-blind placebo-controlled study. J Int Med Res 1984; 12: 198 - 205

190 McGavin CR and the British Thoracic Society Research Committee. Oral $\mathrm{N}$-acetylcysteine and exacerbation rates in patients with chronic bronchitis and severe airway obstruction. Thorax 1985; 40: 832835

191 Meister R. Langzeittherapie mit Acetylcystein Retard-Tabletten bei Patienten mit chronischer Bronchitis. Eine doppelblinde-plazebokontrollierte Studie. Forum Prakt Allg Arzt 1986; 25: 18-22

192 Parr GD, Huitson A. Oral fabrol (oral N-acetylcysteine) in chronic bronchitis. Br J Dis Chest 1987; 81: 341 - 348

193 Rasmussen JB, Glennow C. Reduction in days of illness after long-term treatment with $\mathrm{N}$-acetylcysteine controlled-release tablets in patients with chronic bronchitis. Eur Respir J 1988; 1: 351 - 355

194 Hansen NCG, Skriver A, Brorsen-Ries L et al. Orally administered $\mathrm{N}$-acetylcysteine may improve general well-being in patients with mild chronic bronchitis. Respir Med 1994; 88: 531 - 535

195 Pela R, Calcagni AM, Subiaco $S$ et al. N-ace-tylcysteine reduces the exacerbation rate in patients with moderate to severe COPD. Respiration 1999; 66: 495-500

196 Grandjean EM, Berthet P, Ruffmann R et al. Efficacy of oral long-term $\mathrm{N}$-acetylcysteine in chronic bronchopulmonary disease: A metaanalysis of published double blind, placebo-controlled clinical trials. Clin Therapeutics 2000; 22: 209-221

197 Stey C, Steuer J, Bachmann S et al. The effect of oral N-ace-tylcysteine in chronic bronchitis - a quantitative systematic review. Eur Respir J 1999; 14 (Suppl. 30): 381s

198 Poole PJ, Black PN. Oral mucolytic drugs for exacerbations of chronic obstructive pulmonary disease. BMJ 2001; 322: 1 -6

199 Olivieri D, Zavattini G, Tomasini $G$ et al. Ambroxol for the prevention of chronic bronchitis exazerbations: long term multicenter trial. Respiration 1987; 51 (Suppl. 1): $42-51$

200 Decramer N, Rutten-van Molken M, Dekhuijzen PN et al. Effects of $\mathrm{N}$-acetylcysteine on outcomes in chronic obstructive pulmonary disease (Bronchitis Randomized on NAC Cost-Utility Study, BRONCUS): a randomized placebo-controlled trial. Lancet 2005; 365: $1552-1560$

201 Collet JP, Shapiro S, Ernst $P$ et al. Effect of an immunostimulating agent on acute exacerbations and hospitalizations in COPD patients. Am J Respir Crit Care Med 1997; 156: 1719- 1724

202 Li J, Zheng HP, Yuan JP et al. Protective effect of a bacterial extract against acute exacerbations in patients with chronic bronchitis accompanied by chronic obstructive pulmonary disease. Chin Med J 2004; 173: 4164-4170

203 Anthonisen NR. Editoral. OM-85BV for COPD. Am J Respir Crit Care Med 1997; 156: $1713-1714$

204 Irwin RS, Boulet LP, Cloutier MM et al. Managing cough as a defense mechanism and as a symptom: a consensus panel report of the American College of Chest Physicans. Chest 1998; 114: 133S-181S

205 Bardsley PA, Howard P, DeBacker W et al. Two years treatment with almitrine bismesylate in patients with hypoxic chronic obstructive airways disease. Eur Respir J 1991; 4: 308-310

206 Watanabe S, Kanner RE, Cutillo AG et al. Long-term effect of almitrine bismesylate in patients with hypoxemic chronic obstructive pulmonary disease. Am J Respir Dis 1989; 140: 1269- 1273

207 Winkelmann BR, Kullmer TH, Kneissl DG et al. Low-dose almitrine bismesylate in the treatment of hypoxemia due to chronic obstructive pulmonary disease. Chest block; 105: 1383-1391
208 Eiser N, Denman WT, West C et al. Oral diamorphine: lack of effect on dyspnoea and exercise tolerance in the "pink puffer" syndrome. Eur Respir J 1991; 4: 926-931

209 Young IH, Daviskas E, Keena VA. Effect of low dose nebulised morphine on exercise endurance in patients with chronic lung disease. Tho$\operatorname{rax} 1989 ; 44: 387-390$

210 Rice KL, Kronenberg RS, Hedemark LL et al. Effects of chronic administration of codeine and promethazine on breathlessness and exercise tolerance in patients with chronic airflow obstruction. $\mathrm{Br} J$ Dis Chest 1987; 81: 287-292

211 Woodcock AA, Gross ER, Gellert AA et al. Effects of dihyrocodeine, alcohol and caffeine on breathlessness and exercise tolerance in patients with chronic obstructive lung disease and normal blood gases. N Engl J Med 1981; 305: 1611 - 1616

212 Poole PJ, Veale AG, Black PN. The effect of sustained release morphine on breathlessness and quality of life in severe chronic obstructive pulmonary disease. Am J Respir Crit Care Med 1998; 157: 1877 - 1880

213 Schönhofer B, Suchi S, Haidl P et al. Eine Epidemiologie zum Stellenwert von oral appliziertem Morphium als Therapieform des schwergradigen Lungenemphysems vom Pink-Puffer-Typ. Med Klin 2001; 96: $325-330$

214 Alpha-1-Antitrypsin Deficiency Registry Group. Survival and FEV1 decline in individuals with severe deficiency of a1-antitrypsin. Am J Respir Crit Care Med 1998; 158: 49-59

215 Seersholm N, Kokjensen A. Clinical features and prognosis of life time non-smokers with severe a1-antitrypsin deficiency. Thorax 1998; 53: $265-268$

216 Wencker M, Banik N, Buhl R. Long-term treatment of a1-antitrypsin deficiency related pulmonary emphysema with human a1-antitrypsin. Eur Respir J 1998; 11: $428-433$

217 Incalci RA, Caradonna P, Ranieri P et al. Correlates of osteoporosis in chronic obstructive pulmonary disease. Respir Med 2000; 94: 10791084

218 American College of Rheumatology ad hoc Committee on glucocorticoid-induced osteoporosis. Recommendations for the prevention and treatment of glucocortocoid-induced osteoporosis. Arthritis Rheumatism 2001; 44: 1496-1503

219 Chavannes N, Vollenberg JJ, Schayck CP van et al. Effects of physical activity in mild to moderate COPD: a systematic review. Br J Gen Pract 2002; 52: $547-548$

220 Berry MJ, Rejeski WJ, Adair NE et al. Exercise rehabilitation and chronic obstructive pulmonary disease stage. Am J Respir Crit Care Med 1999; 160: 1248 - 1253

221 ACCP/AAC/VPR Pulmonary Rehabilitation Guidelines Panel. Pulmonary Rehabilitation. Chest 1997; 112: 1363 - 1395

222 American Thoracic Society. Pulmonary Rehabilitation 1999. Am J Respir Crit Care Med block; 159: 1666-1682

223 Lacasse Y, Wong E, Guyatt GH et al. Meta-analysis of respiratory rehabilitation in chronic obstructive pulmonary disease. Lancet 1996; 348: $1115-1119$

224 Worth H, Meyer A, Folgering $H$ et al. Empfehlungen der Deutschen Atemwegsliga zum Sport und körperlichen Training bei Patienten mit obstruktiven Atemwegserkrankungen. Pneumologie 2000; 54: $61-67$

225 Ringbaek TJ, Broendum E, Hemmingsen L et al. Rehabilitation of patients with chronic obstructive pulmonary disease. Exercise twice a week is not sufficient! Respir Med 2000; 94: 150-154

226 Puente-Maestu L, Sanz ML, Sanz P et al. Comparison of effects of supervised versus self-monitored training programmes in patients with chronic obstructive pulmonary disease. Eur Respir J 2000; 5: $517-525$

227 Lacasse Y, Brosseau L, Milne S et al. Pulmonary rehabilitation for chronic obstructive pulmonary disease. Cochrane Database Syst Rev 2002: CD003793

228 Salman GF, Mosier MC, Beasley BW et al. Rehabilitation for patients with chronic obstructive pulmonary disease: meta-analysis of randomized controlled trials. J Gen Intern Med 2003; 18: 213-221

229 Puhan MA, Schunemann HJ, Frey M et al. How should COPD patients exercise during respiratory rehabilitation? Comparison of exercise modalities and intensities to treat skeletal muscle dysfunction. Tho$\operatorname{rax} 2005$; 60: 367-375

230 American Thoracic Society/European Respiratory Society. Statement on Pulmonary Rehabilitation. Am J Respir Crit Care Med 2006; 173: $1390-1413$ 
231 Bernhard S, Whittom F, Leblanc P et al. Aerobic and strength training in patients with chronic obstructive pulmonary disease. Am J Respir Crit Care Med 1999; 159: 896-901

232 Belman MJ, Botnick WC, Nathan SD et al. Ventilatory load characteristics during ventilatory muscle training. Am J Respir Crit Care Med 1994; 149: 925 - 929

233 Griffiths TL, Burr ML, Campbell IA et al. Results at 1 year of out-patient multidisciplinary pulmonary rehabilitation. Lancet 2000; 355: 362 368

234 Foglio K, Bianchi L, Bruletti G et al. Long-term effectiveness of pulmonary rehabilitation in patients with chronic airways obstruction. Eur Respir J 1999; 13: 125-132

235 Young $P$, Dewse $M$, Ferguson $W$ et al. Improvements in outcomes for chronic obstructive pulmonary disease (COPD) attributable to a hospital-based respiratory rehabilitation programme. Aus NZ J Med 1999; 29: 59-65

236 Dhein Y, Birkenmaier A, Otte B et al. Evaluation of a structured education programme (AFBE) for patients with mild to moderate COPD under outpatient conditions. Am J Respir Crit Care Med 2002; 165: A420

237 Worth $H$, Dhein $Y$. Does patient education modify behaviour in the management of COPD? Patient Educ Couns 2004; 52: 267- 270

238 Bourbeau J, Julien M, Maltais F et al. Reduction of hospital utilization in patients with chronic obstructive pulmonary disease: a diseasespecific self-management intervention. Arch Intern Med 2003; 163: $585-591$

239 Gadoury MA, Schwarztmann K, Rouleau M et al. Self-management reduces both short- and long-term hospitalisation in COPD. Eur Respir J 2005; 26: $853-857$

240 Steier J, Petro W. Physikalische Therapie bei COPD - Evidence based Medicine? Pneumologie 2002; 556: 388 - 396

241 Bekkering GE, Hendriks HJM, Chadwick-Straver RVM et al. Clinical practice guidelines for physical therapy in patients with chronic obstructive pulmonary disease. 2003 Available from. http://www.fysionet.nl, V-03/2003/US

242 Murray JF. The ketch-up bottle method. N Engl J Med 1979; 300: $1155-1157$

243 Anthonisen $P$, Ries $P$, Sogaard-Andersen $T$. The value of lung physiotherapy in the treatment of acute exacerbations in chronic bronchitis. Acta Med Scand 1964; 145: 715 - 719

244 Graham W, Bradley GA. Efficacy of chest physiotherapy and intermittent positive-pressure breathing in the resolution of pneumonia. $\mathrm{N}$ Engl J Med 1978; 299: 624-627

245 King M, Philips DM, Gron D et al. Enhanced tracheal mucus clearance with high frequency chest wall percussion. Am Rev Respir Dis 1983; 128: $511-515$

246 Pryer JA. Physiotherapy for airway clearance in adults. Eur Respir J 1999; 14: $1418-1424$

247 Hietpas $B G$, Roth $R D$, Jensen $M W$. Huff coughing and airway patency. Respir Care 1979; 24: 710-713

248 Cegla UH, Petzow A. Physiotherapie mit dem VRP1 bei chronisch obstruktiven Atemwegserkrankungen - Ergebnisse einer multizentrischen Vergleichsstudie. Pneumologie 1993; 47: 636-639

249 App EM, Wunderlich MO, Lohse P et al. Oszillierende Physiotherapie bei Bronchialerkrankungen - rheologischer- und antientzündlicher Effekt. Pneumologie 1999; 53: 1 - 12

250 Cegla UH, Bautz M, Fröde G et al. Physiotherapie bei Patienten mit COPD und tracheobronchialer Instabilität - Vergleich zweier oszillierender PEP-Systeme (RC-Cornet, VRP1-Destin). Pneumologie 1997; 51: 129-136

251 King M, Feng W, Deng WW et al. Short-term efficacy of RC-Cornet in decreasing cohessiveness of sputum in COPD-patients. Chest 1998; 225: 318

252 Engelken MPKJ, Schols AMWJ, Baker WC et al. Nutritional depletion in relation to respiratory and peripheral muscle function in outpatients with COPD. Eur Respir J 1994; 7: 1793 - 1797

253 Schols AMWJ, Soeters PB, Dingemans AMC et al. Prevalence and characteristics of nutritional depletion in patients with stable COPD eligible for pulmonary rehabilitation. Am Rev Respir Dis 1993; 147: 1187 - 1196

254 Wilson DO, Rogers RM, Wright EC et al. Body weight in chronic obstructive pulmonary disease. The National Institutes of Health intermittend positive-pressure breathing trial. Am Rev Respir Dis 1989; 139: $1435-1438$
255 Wouters EFM, Schols AMWJ. Nutritional depletion in COPD. Eur Respir Rev 1997; 7: 60-65

256 Fitting JW. Nutritional support in chronic obstructive lung disease. Thorax 1992; 47: 141 - 143

257 Hunter ABN, Carey MA, Lorch HW. The nutritional status of patients with chronic obstructive pulmonary disease. Am Rev Respir Dis 1985; $132: 1347$ - 1365

258 Gray-Donald K, Gibbone L, Shapiro SH et al. Nutritional status and mortality in chronic obstructive pulmonary disease. Am J Respir Crit Care Med 1996; 153: 961 - 966

259 Baarends EM, Schols AMWJ, Mostert $R$ et al. Peak exercise in relation to tissue depletion in patients with chronic obstructive pulmonary disease. Eur Respir J 1997; 10: 2807 - 2813

260 Shoup R, Dalshy G, Warner G et al. Body composition and health related quality of life in patients with chronic obstructive airways disease. Eur Respir J 1997; 10: 1576-1580

261 Wijkstra PJ, Atena $R$ van, Kraan J et al. Quality of life in patients with chronic obstructive pulmonary disease improve after rehabilitation at home. Eur Respir J 1994; 7: 307 - 311

262 Ferreira IM, Brooks D, Lacasse Y et al. Nutritional supplementation for stable chronic obstructive pulmonary disease. Cochrane Database Syst Rev 2005; block: CD000998

263 Efthimiou J, Fleming J, Gomes C et al. The effect of supple-mentary oral nutrition in poorly nourished patients with chronic obstructive pulmonary disease. Am Rev Respir Dis 1988; 137: 1075 - 1082

264 Rogers RM, Donahue M, Constantino J. Physiologic effects of oral supplemental feeding in malnourished patients with chronic obstructive pulmonary disease patients. Am Rev Respir Dis block; 145: $1511-1597$

265 Whittaker JS, Buckley CF, Road JD. The effects of re-feeding on peripheral and respiratory muscle function in malnourished chronic obstructive pulmonary disease patients. Am Rev Respir Dis 1990; 142: $283-288$

266 Schols AM, Soeters PB, Mostert R et al. Physiologic effects of nutritional support and anabolic steroids in patients with chronic obstructive pulmonary disease. A placebo-controlled randomized trial. Am J Respir Crit Care Med 1995; 152: 1268-1274, 204

267 Creutzberg EC, Wouters EF, Mistert $R$ et al. A role for anabolic steroids in the rehabilitation of patients with COPD? A double-blind, placebo-controlled, randomized trial. Chest 2003; 124: 1733-1742

268 Casaburi R, Bhasin S, Cosentino L et al. Effects of testestorone and resistance training in men with chronic obstructive pulmonary disease. Am J Respir Crit Care Med 2004; 170: 870-878

269 Bergmann KC, Fischer J, Schmitz M et al. Die stationäre Rehabilitation für Erwachsene. Zielsetzung - Diagnostik und therapeutische Standards - Forschungsbedarf. Pneumologie 1997; 51: 523 - 532

270 Donner CF, Muir JF. Selection criteria and programmes for pulmonary rehabilitation in COPD patients. Rehabilitation and chronic care scientific group of the european respiratory society. Eur Respir J 1997; 10: $744-757$

271 British Thoracic Society. Pulmonary rehabilitation. Thorax 2001; 56 $827-834$

272 Young $P$, Dewse $M$, Fergusson $W$ et al. Respiratory rehabilitation in chronic obstructive pulmonary disease: predictors of non-adherence. Eur Respir J 1999; 13: 867 - 872

273 Connors AF, Dawson NV, Thomas $C$ et al. Outcomes following acute exacerbation of severe chronic obstructive lung disease. The SUPPORT investigators (Study to Unterstand Prognoses and Preferences for Outcomes and Risk of Treatments). Am J Respir Crit Care Med 1996; 154: 959-967

274 Garcia-Aymerich J, Farrero E, Felez MA et al. Risk factors of readmission to hospital for a COPD exacerbation: a prospective study. Thorax 2003; 58: 100 - 105

275 Puhan MA, Scharplatz M, Troosters $T$ et al. Respiratory rehabilitation after acute exacerbation of COPD may reduce risk for readmission and mortality - a systematic review. Respir Res 2005; 6: 54

276 Man WD, Polkey MI, Donaldson N et al. Community pulmonary rehabilitation: a randomised controlled study. BMJ 2004; 329: 1209

277 Schultz K, Bergmann KC, Kenn K et al. Effektivität der pneumologischen Anschluss-Rehabilitation (AHB): Ergebnisse einer multizentrischen prospektiven Beobachtungsstudie. Dtsch Med Wochenschr 2006; 131: 1793 - 1798

278 World Health Organization (WHO). International Classification of Functioning, Disability and Health (ICF). Geneva, 2001: http://www.3.who.int/icf/icftemplate.cfm) 
279 Richtlinien des Gemeinsamen Bundesausschusses über Leistungen zur medizinischen Rehabilitation (Rehabilitations-Richtlinien) nach §92 Abs. 1 Satz 2 Nr. 8 SGB V in der Fassung vom 16. März 2004 (BAnz. S. 6769) vom 31. März 2004 in Kraft getreten am 1. April 2004. 2004 (cited: 2005 Jul 14) Available from: http://www.aerzteblatt.de/ v4/archiv/artiekl.asp?id = 41621),

280 Pulmonary rehabilitation: joint ACCP/AACVPR evidence-based guidelines. ACCP/AACVPR Pulmonary Rehabilitation Guidelines Panel. Chest 1997; 112: 1363 - 1396

281 Verband deutscher Rentenversicherungsträger(VDR), Reha-Kommission. Krankheitsspezifsiche Konzepte. In: VDR, Reha-Kommission (eds). Kommission zur Weiterentwicklung der Rehabilitation in der gesetzlichen Rentenversicherung.Abschlussberichte.Band III, Teilband 2 Frankfurt: VDR, 1991: 483-484, 326

282 Bundesversicherungsanstalt für Angestellte (BfA). Leitlinien zur Rehabilitationsbedürftigkeit bei Krankheiten der Atmungsorgane - für den Beratungsärztlichen Dienst der BfA. 2004 (cited: 2005 Aug 12). Available from: http://www.bfa.de/nn 5910/de/Inhalt/Publikationen/Sonstige/Infos 20f C3 BCr 20 C3 84r zte Dokumente/Reha-Bed C3 Bcrftigkeit 20 2F 20 Pneumologie.html),

283 Medizinischer Dienst der Spitzenverbände der Krankenkassen e.V. (MDS). Begutachtungs-Richtlinien Vorsorge und Rehabilitation vom 12. März 2001. 2001 (cited: 2005 Aug 12). Available from: http://informed.-msd-ev.de/sindbad.nsf/

0/1885f053c9a0c58500256a31005377e7?OpenDocument,

284 Szczepanski R, Taube K, Junggeburth J et al. Ambulante wohnortnahe pneumologische Prävention und Rehabilitation bei obstruktiven Atemwegserkrankungen - Stand, Perspektive und Forschungsbedarf. Pneumologie 1998; 52: 476 - 482

285 Goldstein RS, Gort EH, Stubbing D et al. Randomized controlled trial of respiratory rehabilitation. Lancet 1994; 344: 1394-1397

286 McGavin CR, Gapta SP, Lloyd EL et al. Physical rehabilitation for the chronic bronchitis. Results of a controlled trial of exercises in the home. Thorax 1977; 32: 307 - 311

287 Magnussen H, Goeckenjan G, Köhler D et al. Leitlinien zur SauerstoffLangzeit-Therapie. Pneumologie 2001; 55: 454-464

288 Report of the Medical Research Council Working Party. Long-term domiciliary oxygen in chronic cor pulmonale complicating chronic bronchitis and emphysema. Lancet 1981; 1: 681-685

289 Nocturnal oxygen therapy trial group. Continuous or nocturnal oxygen therapy in hypoxemic chronic obstructive pulmonary disease. Ann Intern Med 1980; 93: 191 - 198

290 Weitzenblum E, Sautegeau A, Ehrhart M et al. Long-term oxygen therapy can reverse the progression of pulmonary hypertension in patients with chronic obstructive pulmonary disease. Am Rev Respir Dis 1985; 131: $493-498$

291 Leach RM, Davidson AC, Chinn S et al. Portable liquid oxygen and exercise ability in severe respiratory disability. Thorax 1992; 47: 78 - 789

292 Tarpy SP, Celli BR. Long-term oxygen therapy. N Engl J Med 1995; 333: $710-714$

293 Pelletier Fleury N, Laone JL, Fleury B et al. The cost of treating COPD patients with long-term oxygen therapy in a French population. Chest 1996; 110: $411-416$

294 Heaney LG, McAllister D, MacMahon J. Cost minimisation analysis of provision of oxygen at home: are the drug tariff guidelines cost effective? BMJ 1999; 319: 19-23

295 Donahoe M, Rogers RM. Nutritional assessment and support in chronic pulmonary disease. Clin Chest Med 1990; 11: 487-504

296 Köhler D, Criée CP, Raschke F. Leitlinien zur häuslichen Sauerstoffund Heimbeatmungstherapie. Pneumologie 1996; 50: 927-931

297 Simmonds AK, Elliot MW. Outcome of domiciliary nasal intermittent positive pressure ventilation in restrictive and obstructive disorders. Thorax 1995; 50: 604-609

298 Meecham Jones DJ, Paul EA, Jones PW et al. Nasal pressure support ventilation plus oxygen compared with oxygen therapy alone in hypercapnic COPD. Am J Respir Crit Care Med 1995; 152: 538 - 544

299 Consensus Conference. Clinical indications for noninvasive positive pressure ventilation in chronic respiratory failure due to restrictive lung disease, COPD, and nocturnal hypoventilation. A consensus conference report. Chest 1999; 116: 521 - 534

300 Mehran RJ, Deslauriers J. Indications for surgery and patient work-up for bullectomie. Chest Surg Clin N Am 1995; 5: 717 - 734

301 Hughes JA, Hutchison DM, MacArthur DCS et al. Long-term changes in lung function after surgical treatment of bullos emphysema in smokers and exsmokers. Thorax 1986; 39: 140
302 Cooper JD, Trulock EP, Triantafillou AN et al. Bilateral pneumonectomy (volume reduction) for chronic obstructive pulmonary disease. J Thorac Cardiovasc Surg 1995; 109: 106 - 119

303 National Emphysema Treatment Trial Research Group. A randomized trial comparing lung-volume reduction surgery with medical therapy for severe emphysema. N Engl J Med 2003; 348: 2059-2073

304 Crouch R, Fishman AP, Ries AL et al. The Effects of Pulmonary Rehabilitation in the National Emphysema Treatment Trial. Chest 2005; 128: 3799 - 3809

305 Venuta F, Yim APC, Wan IYP et al. Bronchoscopic Lung Volume Reduction for End-Stage Emphysema: Report on the First 98 Patients. Chest 2006; 129: $518-526$

306 Trulock EP, Edwards LB, Taylor DO et al. The registry of the International Society for Heart and Lung Transplantation: Twenty-first Official Adult Lung and Heart-Lung Transplant Report. J Heart Lung Transplant 2005; 24 : 956-967

307 Deutsche Stiftung Organtransplantation (DSO). Bericht über die Tätigkeit der Transplantationszentren sowie die Organspende in der Bundesrepublik Deutschland. http://www.dso.de/pdf/berichte2005/Gesamt_Taetigkeitsbericht2005.pdf,

308 Hosenpud JD, Bennett LE, Keck BM et al. Effects of diagnosis on survival benefit of lung transplantation for end-stage lung disease. Lancet 1998; $3: 24-27$

309 Charman SC, Sharples LD, McNeil KD et al. Assessment of Survival Benefit After Lung Transplantation by Patient Diagnosis. J Heart Lung Transplant 2002; 21: 226-232

310 Gottlieb J, Simon A, Welte T. Lungentransplantation. Pneumologe 2005; 2: $131-141$

311 Machado MC, Krishnan JA, Buist SA et al. Sex differences in survival of oxygen-dependent patients with chronic obstructive pulmonary disease. Am J Respir Crit Care Med 2006 Sep 1; 174 (5): 524-529

312 Orens JB, Estenne M, Arcasoy $S$ et al. International Guideline for the selection of lung transplant candidates: 2006 Update - A consensus report from the pulmonary scientific council of the international society for heart and lung transplant. J Heart Lung Transplant 2006; 25: $745-755$

313 Meyer DM, Bennett LE, Novick RJ et al. Single vs bilateral, sequential lung transplantation for end-stage emphysema: influence of recipient age on survival and secondary end-points. J Heart Lung Transplant 2001 Sep; 20 (9): 935 - 941

314 Trulock EP, Edwards LB, Taylor DO et al. Registry of the International Society for Heart and Lung Transplantation: twenty-third official adult lung and heart-lung transplantation report - 2006. J Heart Lung Transplant 2006; 25: 880-892

315 Elkington $H$, White $P$, Addington-Hall $J$ et al. The last year of life of COPD: a qualitative study of symptoms and services. Respir Med 2004; 98: $439-445$

316 Booth $S$, Wade R, Johnson $M$ et al. The use of oxygen in the palliation of breathlessness. A report of the expert working group of the Scientific Committee of the Association of Palliative Medicine. Respir Med 2004; 98: $66-77$

317 Jennings $A L$, Davies AN, Higgins JP et al. A systematic review of the use of opioids in the management of dyspnoea. Thorax 2002; 57: 939 944

318 Steinkamp G, Dierkesmann R, Gillissen A et al. COPD und Psyche - ein Überblick. Pneumologie 2005; 59: 819-830

319 World Health Organization. Cancer pain relief and palliative care - report of a WHO expert committee. Genf: World Health Organization, 1990

320 Anthonisen NR, Manfreda J, Warren CP et al. Antibiotic therapy in exacerbations of chronic obstructive pulmonary disease. Ann Intern Med 1987; 106: $196-204$

321 Regueiro CR, Hamel MB, Davis RB et al. A comparison of generalist and pulmonologist care for patients hospitalized with severe chronic obstructive pulmonary disease: resource intensity, hospital costs and survival. Am J Med 1998; 105: 366 - 372

322 Gibson PG, Wlodarczyk JH, Wilson AJ et al. Severe exacerbations of chronic obstructive airways disease: health resource use in general practice and hospital. J Qual Clin Pract 1998; 18: 125 - 133

323 Mushlin AI, Black ER, Connolly CA et al. The necessary length of hospital stay for chronic pulmonary disease. JAMA 1991; 266: 80-83

324 Connors AF, Dawson NV, Thomas C et al. Outcomes following acute exacerbation of severe chronic obstructive lung disease. The SUPPORT investigators. Am J Respir Crit Care Med 1996; 154: 959-967 
325 Kong GK, Belman MJ, Weingarten S. Reducing length of stay for patients hospi-talized with exacerbation of COPD by using a practice guideline. Chest 1997; 111: 89 - 94

326 Fuso L, Incalzi RA, Pistelli R et al. Predicting mortality of patients hospitalized for acutely exacerbated chronic obstructive pulmonary disease. Am J Med 1995; 98: 272 - 277

327 Seneff MG, Wagner DP, Wagner RP et al. Hospital and 1-year survival of patients admitted to intensive care units with acute exacerbation of chronic obstructive pulmonary disease. JAMA 1995; 274: 1852 1857

328 MacFarlane JT, Colville A, Macfarlane RM et al. Prospective study of aetiology and outcome of adult lower-respiratory-tract infections in the community. Lancet 1993; 341: $511-514$

329 Stockley RA, O'Brien C, Pye A et al. Relationship of sputum color to nature and outpatient management of acute exacerbations of COPD. Chest 2000; 117: $1638-1645$

330 Seemungal TR, Harpen-Owen R, Bhownik A et al. Respiratory viruses, symptoms and inflammatory markers in acute exacerbations and stable chronic obstructive pulmonary disease. Am J Respir Crit Care Med 2001; 164: 1618 - 1623

331 Anderson HR, Spix C, Medina S et al. Air pollution and daily admissions for chronic obstructive pulmonary disease in 6 European cities: results from the APHEA project. Eur Respir J 1997; 10: 1064-1071

332 Emerman CL, Connors AF, Lukens TW et al. Relationship between arterial blood gases and spirometry in acute exacerbations of chronic obstructive pulmonary disease. Ann Emerg Med 1989; 18: 523- 527

333 Emerman CL, Effron D, Lukens TW. Spirometric criteria for hospital admission of patients with acute exacerbation of COPD. Chest 1991; 99: $595-599$

334 Emerman CL, Lukes TW, Effron D. Physician estimation of FEV1 in acute exacerbation of COPD. Chest 1994; 105: 1709-1712

335 Emerman CL, Cydulka RK. Use of peak expiratory flow rate in emergency depart- ment evaluation of acute exacerbation of chronic obstructive pulmonary disease [see comments]. Ann Emerg Med 1996: 27: $159-163$

336 Tillie-Leblond I, Marquette $\mathrm{CH}$, Perez T et al. Pulmonary embolism in patients with unexplained exacerbation of chronic obstructive pulmonary disease: prevalence and risk factors. Ann Intern Med 2006; 144: $930-936$

337 Rice KL, Leatherman JW, Duane PG et al. Aminophylline for acute exacerbations of chronic obstructive pulmonary disease. A controlled trial. Ann Intern Med 1987; 107: 305 - 309

338 Barbera JA, Reyes A, Roca J et al. Effect of intravenously administered aminophylline on ventilation/perfusion inequality during recovery from exacerbations of chronic obstructive pulmonary disease. Am Rev Respir Dis 1992; 145: 1328 - 1333

339 Mahon JL, Laupacis A, Hodder RV et al. Theophylline for irreversible chronic airflow limitation: a randomized study comparing $\mathrm{n}$ of 1 trials to standard practice. Chest 1999; 115: $38-48$

340 Loberes P, Ramis L, Montserrat JM et al. Effect of three different bronchodilators during an exacerbation of chronic obstructive pulmonary disease. Eur Respir J 1988; 1: 536 - 539

341 Emerman CL, Connors AF, Lukens TW et al. Theophylline concentrations in patients with acute exacerbation of COPD [see comments]. Am J Emerg Med 1990; 8: 289-292

342 Duffy $N$, Walker P, Diamantea $F$ et al. Intravenous aminophylline in patients admitted to hospital with non-acidotic exacerbations of chronic obstruktive pulmonary disease: a prospective randomized controlled trial. Thorax 2005; 60: $713-717$

343 Christ-Crain M, Jaccard-Stolz D, Bingisser $R$ et al. Effect of procalcitonin-guided treatment on antibiotic use and outcome in lower respiratory tract infections: cluster-randomised, single-blinded intervention trial. Lancet 2004; 363 (9409): 600-607

344 Nouira S, Marghli S, Belghith M et al. Once daily oral ofloxacin in chronic obstructive pulmonary disease exacerbation requiring mechanical ventilation: a randomised placebo-controlled trial. Lancet 2001; 358: $2020-2025$

345 Worth $H$, Adam D, Handrick W et al. Prophylaxe und Therapie von bronchopulmonalen Infektionen. Empfehlungen der Deutschen Atemwegsliga. Pneumologie 1998; 52: 232 - 237

346 Welte T, Marre R, Suttorp N. Kompetenznetzwerk „Ambulant Erworbene Pneumonie“ (CAPNETZ). Related Articles, What is new in the treatment of community-acquired pneumonia? Med Klin (Munich) 2006 Apr 15; 101 (4): 313 - 320

347 Arancibia F, Bauer TT, Ewig S et al. Community-acquired Pneumonia caused by gram-negative bacteria: Incidence and risk and prognosis. Arch Intern Med 2002; 162 (16): 1849-1858

348 Thompson, WH, Nielson CP, Carvalho P et al. Controlled trial of oral prednisolone in outpatients with acute COPD exacerbation. Am J Respir Crit Care Med 1996; 154: 407 - 412

349 Davies L, Angus RM, Calverley PM. Oral corticosteroids in patients admitted to hospital with exacerbations of chronic obstructive pulmonary disease: a prospective randomised controlled trial. Lancet 1999; 354: $456-460$

350 Niewoehner DE, Erbland ML, Deupree RH et al. Effect of systemic glucocorticoids on exacxerbations of chronic obstructive pulmonary disease. N Engl J Med 1999; 340: 1941 - 1947

351 Bach PB, Brown C, Gelfand SE et al. Management of acute exacerbations of chronic obstructive pulmonary disease: a summary and appraisal of published evidence. Ann Intern Med 2001; 134: 600 620

352 Rossi A, Gottfried SB, Zocci L et al. Respiratory mechanics in mechanically ventilated patients with respiratory failure. J Appl Physiol 1985; 58: $1849-1858$

353 Georgopoulos D, Rossi A, Moxham J. Ventilatory support in chronic obstructive pulmonary disease. Eur Respir Mon 1998; 7: 189-208

354 Bott J, Carroll MP, Conway JH et al. Randomized controlled trial of nasal ventilation in acute ventilatory failure due to chronic obstructive airways disease. Lancet 1993; 341: 1555-1557

355 Brochard L, Mancebo J, Wysocki $M$ et al. Noninvasive ventilation for acute exacerbations of chronic obstructive pulmonary disease. N Engl J Med 1995; 33: 817 - 822

356 Kramer N, Meyer TJ, Meharg J et al. Randomized, prospective trial of noninvasive positive pressure ventilation in acute respiratory failure. Am J Respir Crit Care Med 1995; 151: 1799-1806

357 Barbeacute F, Togores B, Rubi M et al. Noninvasive ventilatory support does not facilitate recovery from acute respiratory failure in chronic obstructive pulmonary disease. Eur Respir J 1996; 9: 1240-1245

358 Plant PK, Owen JL, Elliott MW. Early use of non-invasive ventilation (NIV) for acute exacerbations of COPD on general respiratory wards: a multicenter randomized controlled trial. Lancet 2000; 355: 1931 1935

359 Nava S, Ambrosino N, Clini E et al. Noninvasive mechanical ventilation in the weaning of patients with respiratory failure due to chronic obstructive pulmonary disease. A randomized, controlled trial. Ann Intern Med 1998; 128: 721 - 728

360 Stoller JK, Lange PA. Inpatient management of chronic obstructive pulmonary disease. Respir Care Clin N Am 1998; 4: 425 - 438

361 Friedman M, Serby CW, Menjoge SS et al. Pharmacoeconomic evaluation of a combination of ipratropium plus albuterol compared with ipratropium alone and albuterol alone in COPD. Chest 1999; 115: $635-641$

362 Morgan WKG, Reger RB. Chronic airflow limitation and occupation. In: Cherniack NS (Hrsg). Chronic obstructive pulmonary disease. Philadelphia: Saunders, 1991

363 Nowak D. Berufsbedingte obstruktive Atemwegserkrankung (BK4301 und 4302) gutachterliche Anmerkungen zum Krankheitsbegriff und zur MdE. Arbeitsmed Sozialmed Umweltmed 2000; 35: $164-167$

364 Morfeld P, Piekarski C. Chronische Bronchitis und Emphysem als Berufskrankheit der Steinkohlenbergleute. Schrittenreiche Zentralblatt für Arbeitsmedizin Haefer, 1998

365 Nowak D. Informationen zur neuen Berufskrankheit 4111: „Chronische obstruktive Bronchitis oder Emphysem von Bergleuten unter Tage im Steinkohlenbergbau bei Nachweis der Einwirkung einer kumulativen Feinstaubdosis von in der Regel $100\left[\left(\mathrm{mg} / \mathrm{m}^{3}\right)\right.$ Jahre]“. Pneumologie 1996; 50: $652-654$

366 Kroidl RF, Nowak D, Seysen U. Bewertung und Begutachtung in der Pneumologie. Thieme, 1999

367 Bundesministerium für Arbeit und Sozialordnung. Anhaltspunkte für die Ärztliche Gutachtertätigkeit im sozialen Entschädigungsrecht und nach dem Schwerbehindertengesetz Köllen, 1996 